\title{
SEMI-PARAMETRIC SuRVIVAL Analysis via Dirichlet Process Mixtures of the First Hitting TIME MODEL
}

\author{
DISSERTATION
}

Presented in Partial Fulfillment of the Requirements for the Degree Doctor of Philosophy in the Graduate School of The Ohio State University

By

Jonathan A. Race, B.S.

Doctoral Program in Biostatistics

The Ohio State University 2019

Dissertation Committee:

Michael Pennell, Advisor

Guy Brock

Mario Peruggia

Patrick Schnell 
(c) Copyright by

Jonathan A. Race

2019 


\section{ABSTRACT}

Time-to-event data often violate the proportional hazards assumption inherent in the popular Cox regression model. Such violations are especially common in the sphere of biological and medical data where latent heterogeneity due to unmeasured covariates or time varying effects are common. A variety of parametric survival models have been proposed which make more appropriate assumptions on the hazard function, at least for certain applications. One such model is derived from the First Hitting Time (FHT) paradigm which assumes that a subject's event time is determined by a latent stochastic process reaching a threshold value. Several random effects specifications of the FHT model have also been proposed which allow for better modeling of data with unmeasured covariates. While often appropriate, these methods often display limited flexibility due to their inability to model a wide range of heterogeneities. To address this issue, we propose two Bayesian models which loosen assumptions on the mixing distribution inherent in the random effects FHT models currently in use. The first proposed model is ideally suited for standard regression analyses. The second model is designed for use in clinical trials where survival is the outcome of interest. We demonstrate via simulation study that the proposed models greatly improve both survival and parameter estimation in the presence of latent heterogeneity. We also apply the proposed methodologies to data from a toxicology/carcinogenicity study which exhibits nonproportional hazards and contrast the results with competing methods. 
To my wife who wouldn't let me give up and my children who made the effort worth it. 


\section{ACKNOWLEDGMENTS}

This work is the culmination of several years worth of stimulating research and laborious effort. More importantly, though, this work is a testament to the support, instruction, and guidance which laid the foundation for my success. I would be remiss if I did not acknowledge those individuals who have been most influential in shaping that success.

I would like to begin by expressing profound gratitude to my dissertation advisor, Dr. Michael Pennell, whose mentorship these past three years has been invaluable. I am especially grateful for Dr. Pennell's patience throughout the early dissertation work as I clumsily introduced myself to the world of research. In addition to offering guidance and perspective, Dr. Pennell also provided what all graduate researchers need to succeed: an interesting but approachable problem. I have quite enjoyed my time working on the material in this dissertation and none of it would have been possible without the support and opportunities that Dr. Pennell provided.

The research in this work also owes a great deal of credit to my dissertation committee members: Dr. Mario Peruggia, Dr. Guy Brock, and Dr. Patrick Schnell. In addition to significant manuscript review, each has provided insightful critique which has prompted deeper reflection and resulted in a more complete methodology. I could not have asked for a more helpful committee.

Another academic advisor also deserves my special recognition and gratitude. As an undergraduate mathematics student I had the unique opportunity to work with Dr. Lajos Horvath. Lajos was a demanding mentor, especially for students whom he knew to have great potential. Such a mentor, though, was exactly what I personally needed to succeed. As an instructor Lajos demanded exactly what he knew I was capable of, and nothing less. 
He pushed me to be better when a less interested teacher might have accepted merely good enough. When I was contemplating graduate school I asked Lajos if he would write a letter of recommendation for my application to a masters program. He declined. Lajos knew that I was capable to achieving more and so, as he had done throughout my undergraduate experience, he demanded more. He informed me in no uncertain terms that he would not write a letter of recommendation unless it was for applications to doctoral programs. To that point in my career I had never seriously considered that I might be capable of earning a doctorate degree. It had been a dream, sure, but not a serious one. It took a demanding mentor for me to see my own potential. For that I owe Dr. Horvath a great deal of gratitude.

My professional and academic successes owe a great deal to a supportive family and especially to my parents. They were never shy about saying how much they believed in me even when, or maybe especially when, I struggled to believe in myself. While my parents' support was absolute, they also expected me to take responsibility for my own success. I will never forget the time as a junior high student when my mother refused to talk to a teacher about the possibility of me making up work that I had forgotten to turn in. She told me that I was old enough to be responsible for my own mistakes and that if I wanted to work out an alternative with my teacher, that was my prerogative and it was on my shoulders. Approaching that teacher was one of the most intimidating experiences of my formative years, but he was indeed open to working with me to improve my grades. That lesson in personal responsibility is one that has remained with me ever since and has served me well.

I also owe a profound debt to my Grandpa Crosslin. My love of science and mathematics was first sparked during long-ago nights when Grandpa would let me stay up inordinately late to assist with his amateur astronomy. Seeing Jupiter and Mars and Comet Hyakutake through the lens of Grandpa's telescope filled me with something beyond curiosity or even fascination. I was so enthralled with space that at the ripe old age of 8 I knew I wanted to be an astronomer while my peers all talked about being policemen or firefighters. That dream, of course, was not quite the path the I would ultimately take. But the love of science and the fascination with the universe that I experienced with my Grandpa as a young boy 
have stayed with me since. I may not have become a professional astronomer, but I can absolutely say that I would not be where I am now without my Grandpa Crosslin.

Finally, and most importantly, I need to publicly acknowledge the sacrifices of my dear wife, Mykin. The past five years have been incredibly challenging, with the demands of graduate school often requiring more time and energy than either of us could have anticipated. Early mornings and late nights have been the hallmark of my graduate school experience. Yet as exhausted as I so often was, this endeavor has been just as taxing on Mykin. Far too often she was left to run our home by herself because I was out the door before anyone else was awake and would not be home until well after our children were sound asleep. I am sure there were weeks and even months that Mykin felt as if she were a single parent, doing it all on her own. To her inestimable credit, Mykin always did what needed doing with little fanfare, with no complaint, and with even less expectation of recognition. In truth, though, there is no one more deserving of my recognition and my gratitude. Mykin, my dearest, thank you. 


\section{VITA}

$2014 \ldots \ldots \ldots \ldots \ldots \ldots \ldots \ldots \ldots \ldots \ldots \ldots \ldots \ldots$.S. Mathematics - The University of Utah, Salt Lake City, UT

2014 to 2015 ......................... University Fellow, The Ohio State University, Columbus, $\mathrm{OH}$

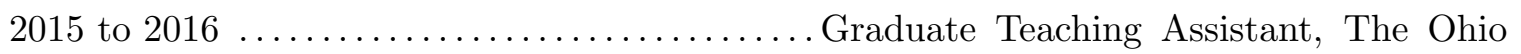
State University, Columbus, $\mathrm{OH}$

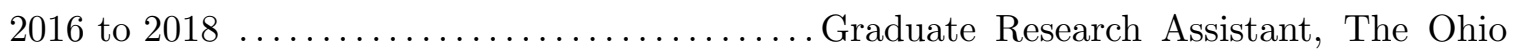
State University, Columbus, $\mathrm{OH}$

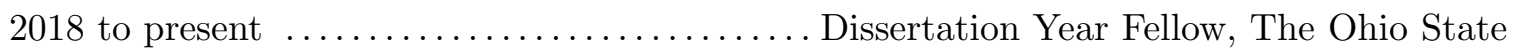
University, Columbus, $\mathrm{OH}$

\section{Fields of Study}

Major Field: Biostatistics

Studies in Bayesian inference, survival analysis, clinical trials, hierarchical and mixed models, Bayesian non- and semi-parametrics.: Dr. Michael L Pennell 


\section{Table of Contents}

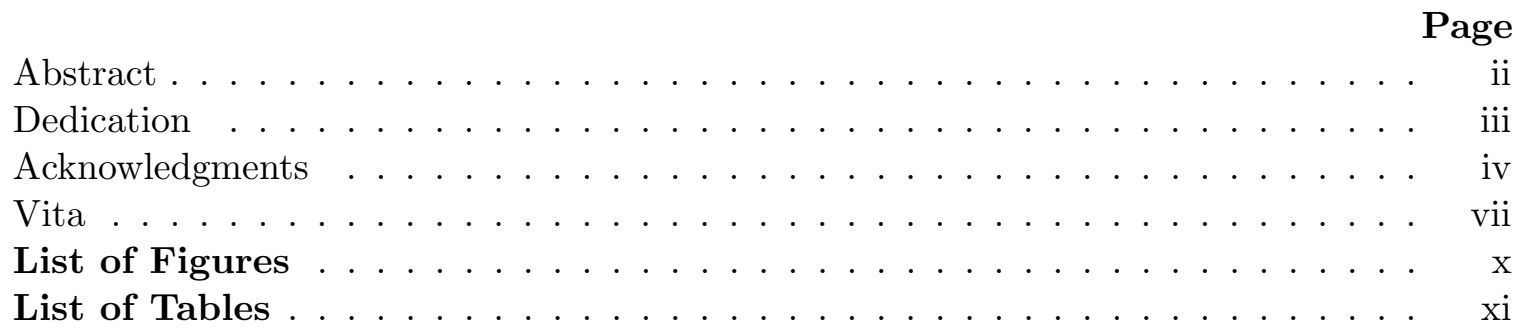

\section{Chapters}

1 Introduction .......................... 1

2 First Hitting Time Models . . . . . . . . . . . . . . . 5

2.1 The Inverse-Gaussian Density . . . . . . . . . . . . . . . . . 6

2.2 The Inverse-Gaussian Distribution as the First Hitting Time of a Wiener Process ........................... 8

2.3 First Hitting Time Models . . . . . . . . . . . . . . . . . . . . . 13

2.3.1 The Hazard Function . . . . . . . . . . . . . . . . . . 15

2.3.2 The Observed Data Likelihood . . . . . . . . . . . . . . . 18

2.4 Threshold Regression Models . . . . . . . . . . . . . . . . . . . . . 19

2.4.1 Threshold Regression: ML Model Fitting . . . . . . . . . . . . . 20

2.4.2 Threshold Regression: Bayesian Modeling . . . . . . . . . . . . . . 20

2.4.3 Threshold Regression: Collinearity . . . . . . . . . . . . . . . . 22

2.5 Heterogeneity in First Hitting Time Models . . . . . . . . . . . . . . . . 24

2.5.1 Random Drift TR Model . . . . . . . . . . . . . . . . . . 24

2.5.2 Random Initial State FHT Model . . . . . . . . . . . . . . 26

2.5.3 Normal-gamma Mixtures of IG Distributions . . . . . . . . . . 28

2.5.4 Bayesian TR with Random Effects . . . . . . . . . . . . . . . 29

2.5.5 Threshold Regression: Finite Mixtures . . . . . . . . . . . . . . 32

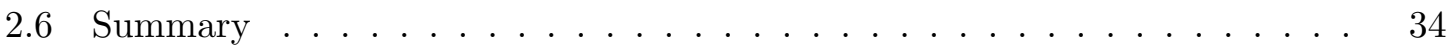

3 The Dirichlet Process Mixture ... . . . . . . . . . . 36

3.1 The Dirichlet Process Mixture Model . . . . . . . . . . . . . . . . 36

3.2 DPMM - Polya-Urn Sampler . . . . . . . . . . . . . . . . 41

3.2.1 Polya-urn Interpretation . . . . . . . . . . . . . . . . . . . . . . . 42

3.2.2 The Polya Urn Gibbs Sampler . . . . . . . . . . . . . . . . . . 42 
3.2.3 MCMC Mixing in the Polya Urn Sampler . . . . . . . . . . . . . 45

3.2.4 Conjugacy in the Polya Urn Sampler . . . . . . . . . . . . . . . . 45

3.3 DPMM - Blocked Gibbs Sampler . . . . . . . . . . . . . . . 46

3.3.1 Stick-Breaking Interpretation . . . . . . . . . . . . . . . . 46

3.3.2 Truncation of the Stick-Breaking Representation . . . . . . . . . . . 47

3.3.3 The Weak Limit Approximation of the DP . . . . . . . . . . . . . 49

3.3.4 Blocked Gibbs Sampler for Approximate DP Mixture Models . . . . 51

3.4 Posterior Inference for $\mathcal{G}$ and its Functionals . . . . . . . . . . . . 56

3.5 Use of the Dirichlet Process in Survival Analysis . . . . . . . . . . . . 57

4 The Dirichlet Process Mixture for Threshold Regression . . . . . . . 59

4.1 The DPM-TR Model . . . . . . . . . . . . . . . . . . . . 60

4.2 Model Selection Criteria . . . . . . . . . . . . . . . . . 63

4.3 The Blocked Sampler . . . . . . . . . . . . . . . . . . . 64

4.4 Simulation Study . . . . . . . . . . . . . . . . . . . . 70

4.4 Simulated Data . . . . . . . . . . . . . . . . 70

4.4 .2 Fitted Models . . . . . . . . . . . . . . . . . . . 72

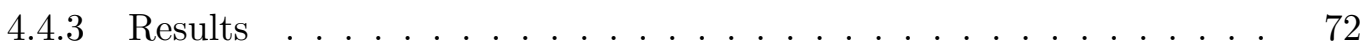

4.5 Data Example . . . . . . . . . . . . . . . . . . . . 80

4.6 Discussion . . . . . . . . . . . . . . . . . . . 85

5 Testing for Ordinal Treatment Effects on Survival via Dynamic Mixture of Dirichlet Processes for Threshold Regression . . . . . . . . . 88

5.1 General DMDP Framework . . . . . . . . . . . . . . . . . . 89

5.1.1 Hypothesis Testing Under the DMDP Model . . . . . . . . . . . . . 91

5.1 .2 Prior Specifications for the DMDP Model . . . . . . . . . . . . 96

5.1.3 Hypothesis Testing Under the DMDP Model . . . . . . . . . . . . . 98

5.2 Dynamic Mixture of Dirichlet Processes for Threshold Regression . . . . . . 100

5.2.1 MCMC Sampler for the DMDP-TR model . . . . . . . . . . . . . 102

5.3 Simulation Study . . . . . . . . . . . . . . . . 107

$5.3 .1 \quad$ Simulated Data . . . . . . . . . . . . . . . . . . 107

5.3 .2 Analysis Methods . . . . . . . . . . . . . . . . . . . . . 119

5.3 .3 Results . . . . . . . . . . . . . . . . . . . . 122

5.4 Data Example. . . . . . . . . . . . . . . . . . . . . . . 129

5.5 Discussion . . . . . . . . . . . . . . . . . . . . 133

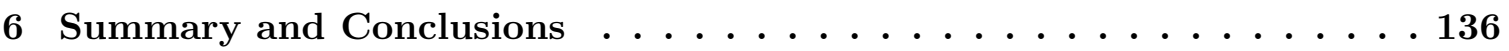

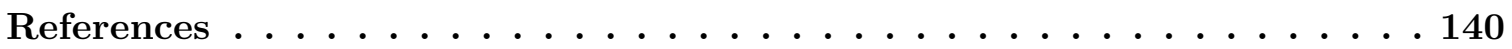

\section{Appendices}




\section{List of Figures}

Figure

Page

2.1 Inverse Gaussian Hazards 1 . . . . . . . . . . . . . . . . . . . 17

2.2 Inverse Gaussian Hazards $2 \ldots \ldots$. . . . . . . . . . . . 18

3.1 Draws from the Dirichlet Process . . . . . . . . . . . . . . . . . . 39

4.1 DPM-TR Simulation Study, survival estimation . . . . . . . . . . . . . . 74

4.2 DPM-TR Simulation Study, bias and RMSE for $\alpha_{1} \ldots \ldots \ldots \ldots$

4.3 DPM-TR Simulation Study, bias and RMSE for $\beta_{1} \ldots \ldots \ldots \ldots$. . . . 77

4.4 DPM-TR Simulation Study, model selection via PML . . . . . . . . . . . 80

4.5 DPM-TR Data Example, estimated survival by treatment group . . . . . . 83

5.1 DMDP-TR Simulation Study, true survival curves for Case 1 . . . . . . . 109

5.2 DMDP-TR Simulation Study, true survival curves for Case 2 . . . . . . . 110

5.3 DMDP-TR Simulation Study, true survival curves for Case 3 . . . . . . . . 112

5.4 DMDP-TR Simulation Study, true survival curves for Case 4(a) . . . . . . . 114

5.5 DMDP-TR Simulation Study, true survival curves for Case 4(b) . . . . . . 116

5.6 DMDP-TR Simulation Study, true survival curves for Case 5 . . . . . . . 117

5.7 DMDP-TR Simulation Study, power and Type 1 error for unconfounded cases 125

5.8 DMDP-TR Simulation Study, power and Type 1 error for confounded cases 126

5.9 DMDP-TR Simulation Study, median survival error for non-confounded cases 127

5.10 DMDP-TR Simulation Study, median survival error for confounded cases . 128

5.11 DMDP-TR Data Example, posterior distribution for $\mu \ldots \ldots$. . . . . . 132

5.12 DMDP-TR Data Example, estimated survival . . . . . . . . . . . . . 133 


\section{List of Tables}

4.1 DPM-TR Data Example, estimated survival agreement . . . . . . . . . . . 83

4.2 DPM-TR Data Example, parameter estimates . . . . . . . . . . . . . . 84

5.1 DMDP-TR Simulation Study, Case 4(b) bias . . . . . . . . . . . . . 117

5.2 DMDP-TR Simulation Study, Censoring model parameters . . . . . . . . 119

5.3 DMDP-TR Data Example, test significance . . . . . . . . . . . . . . . 130

5.4 DMDP-TR Data Example, distribution of LOEL . . . . . . . . . . . . . . 131 


\section{Chapter 1 INTRODUCTION}

Ordinary people think merely of spending time, great people think of using it.

- Arthur Schopenhauer

Analysis of time-to-event data is of wide interest across a variety of disciplines ranging from medicine and public health to engineering and economics. It is difficult to imagine even a single area of human endeavor where questions regarding the pattern of events in time are not of some interest. How many years can I expect to live? At what age do children begin walking? How long is my new phone going to last before it breaks? A rich literature colloquially referred to as survival analysis is concerned with proper methods for answering such questions. The current work seeks to augment this literature by developing several flexible and clinically interpretable models capable of offering novel insights into time-to-event patterns. In order to motivate the proposed methodologies we begin with an abbreviated review of what is likely the most successful and widespread survival analysis model currently in use.

As its name suggests, the Cox proportional hazards $(\mathrm{PH})$ model defines the event time distribution in terms of the hazard function, commonly expressed as

$$
\lambda(t)=\frac{f(t)}{S(t)}
$$

where $f(t)$ is the density function for event time $T$ and $S(t)=1-F(t)$ is the associated survival function. The definition of the hazard in (1.0.1) is often colloquially interpreted as the event rate at time $t$ condition on survival up to time $t$. While this interpretation of the 
hazard function might seem somewhat opaque, it is also quite natural to think about events in time in terms of the rates at which they occur. This is particularly true in fields such as public health where researchers are used to thinking in terms of incidence of disease. Even with this simple conception of the hazard, the fundamental structure of the Cox model can be concisely stated as associating subject-specific hazard functions multiplicatively with covariates. In its simplest form, the Cox PH model is stated symbolically as follows:

$$
\lambda\left(t \mid \mathbf{X}_{i}\right)=\lambda_{0}(t) \exp \left\{\mathbf{X}_{i}^{\top} \boldsymbol{\beta}\right\}
$$

where $\lambda_{0}(t)$ is a "baseline" hazard function, $\mathbf{X}_{i}$ is a set of subject-specific covariates, and $\boldsymbol{\beta}$ are the associated coefficients. When we say that the Cox model is a multiplicative model we mean that, given covariate vectors $\mathbf{X}_{i}$ and $\mathbf{X}_{i}^{*}$ which only differ in term $j$ by $\delta_{j}$ (i.e. $\left.\mathbf{X}_{i}^{*}-\mathbf{X}_{i}=\left(0, \ldots, 0, \delta_{j}, 0, \ldots, 0\right)^{\top}\right)$, then the ratio of the hazard functions is a constant

$$
\frac{\lambda\left(t \mid \mathbf{X}_{i}^{*}\right)}{\lambda\left(t \mid \mathbf{X}_{i}\right)}=\exp \left\{\delta_{j} \cdot \beta_{j}\right\}
$$

The popularity of the Cox model can be understood by considering two of its more obvious benefits. The first of these advantages is rather practical and concerns the natural and intuitive interpretation of model parameters. While the given definition of the hazard function does not align perfectly with the common conception of a "hazard", a clinician is still likely to intuitively understand what is meant when she reads a conclusion such as "The treatment group had an estimated hazard rate twice that of the control group". It would be natural for most researchers to interpret this statement as saying that the treatment group experienced the event twice as often as the control group. While this interpretation is not technically precise, it is sufficient for most clinical purposes and this naturalness has greatly facilitated the adoption of the Cox model throughout biomedical research. The second major advantage of the Cox model, somewhat more theoretical, is the model's relative flexibility due to nonparametric estimation of the baseline hazard $\lambda_{0}(t)$. Stated another way, when one fits the Cox $\mathrm{PH}$ model there is no need to assume that the time-to-event distribution has any particular form. This is quite advantageous from a technical point of view because it lessens dependence on potentially problematic assumptions. 
As with all modeling techniques, however, the Cox $\mathrm{PH}$ model has several significant limitations. The general theme of these limitations relates to the (potentially) strong assumption that hazards are in fact proportional. Violation of the proportionality assumption can occur for a variety of reasons, including time-dependent effects and the presence of unexplained heterogeneity (Aalen, Borgan, \& Gjessing, 2008). Violations of the proportionality assumption can lead to erroneous inference. A large literature exists discussing possible solutions for these issues. Hastie and Tibshirani (1990) and Tian, Zucker, and Wei (2005), for example, discuss methods to address time-dependent covariate effects. Klein and Moeschberger (2003) present stratification methods to address nonproportional hazards. McGilchrist and Aisbett (1991) and Anderson et al. (1997) discuss a fairly broad approach to addressing heterogeneity in $\mathrm{PH}$ models via the inclusion of a frailty term.

Despite the popularity of the Cox PH model and its generalizations, parametric models have also been widely utilized in survival analysis. While the latter generally make stronger assumptions on the hazard and survival functions compared to the former, it is often the case that these stronger assumptions are not only more appropriate for a given situation but are also potentially useful. The First Hitting Time (FHT) model, which forms the foundation for the principal developments of this work, is a prime example of this possibility. Of particular note currently, the FHT model has the somewhat unique ability to explicitly discriminate between covariates whose effect on survival either increases or decreases over time (see Section 2.3.1). While it is possible to accomplish such discrimination utilizing generalizations of the $\mathrm{PH}$ model, to do so is fairly cumbersome. If discrimination between immediate and continuing effects on survival is of scientific interest, abandoning the $\mathrm{PH}$ assumption in favor of the stronger parametric FHT assumptions will often be reasonable.

In spite of the potential benefits of parametric survival models, unmeasured covariates and the resulting latent heterogeneity are just as problematic for the FHT model as they are for $\mathrm{PH}$ models, if not more so. In response, several models employing mixtures of the base FHT model have been proposed which, at least to some extent, account for some types of unmeasured heterogeneity and provide more robust inference (see Sections 2.5.1 2.5.5). Unfortunately, these proposed methods have several important shortcomings related 
to how latent heterogeneity is being modeled. A more flexible methodology for addressing heterogeneity can be found in the Dirichlet Process Mixture literature. We apply Dirichlet Process Mixture ideas to the FHT model in several ways and thereby extend the usefulness of the FHT paradigm in survival analysis.

The remainder of this work is outlined as follows. Chapter 2 will provide a more complete review of the First Hitting Time (FHT) model, including discussions of derivation and interpretation. Chapter 3 will introduce the Dirichlet Process Mixture Model (DPMM). Attention will be particularly focused on conceptual and theoretical developments which will facilitate application of the DPMM methodology to the FHT setting. The principal contributions of this work begin in Chapter 4 where we develop the natural Dirichlet Process Mixture extension of the FHT model. We present results from a simulation study as well as a data application to demonstrate the performance and usefulness of the resulting model. In Chapter 5 we apply the Dirichlet Process prior in a unique manner resulting in a model geared toward analysis of time-to-event data under the assumption of ordinal treatments. Further simulation studies and data examples are reviewed to highlight this second methodology. Chapter 6 summarizes the results from this research and presents a plan for continued refinement and development of the proposed models. This plan is intended to account for the author's early post-doctoral research. 


\section{Chapter 2 \\ First Hitting Time Models}

Don't hit at all if it is honorably possible to avoid hitting; but never hit soft.

- Theodore Roosevelt

The First Hitting Time (FHT) model posits that an observed event is actually the result of an underlying, unobserved stochastic process. Death, for instance, might be conceptualized as the event which occurs when a subject's unknown health process first reaches 0 . If we are willing to make a few basic assumptions about the general behavior of this latent causal process, we can develop a concrete and clinically meaningful model for survival. In the current work we will limit ourselves to modeling assumptions which give rise to an event-time density know as the Inverse-Gaussian distribution. The current chapter will begin with a review of these basic assumptions and several interesting features of the resulting distribution. This discussion will largely draw on the work of Chhikara and Folks (1989) who provide a comprehensive review of the theoretical underpinnings of the InverseGaussian distribution. We then review several results which will assist in developing a clinically insightful parameterization for the FHT model. A discussion of FHT regression models naturally follows and, finally, we will conclude this chapter with a review of several extensions of the FHT framework which allow for explicit modeling of heterogeneity due to unmeasured covariates. 


\subsection{The Inverse-Gaussian Density}

Much of the early research concerning the Inverse Gaussian (IG) distribution focused principally on the mean-precision parameterization presented by Tweedie (1957)

$$
f_{X}(x \mid \nu, \lambda)=\sqrt{\frac{\lambda}{2 \pi}} x^{-3 / 2} \exp \left(-\frac{\lambda(x-\nu)^{2}}{2 \nu^{2} x}\right) I(x>0),
$$

where $\nu>0$ and $\lambda>0$ act respectively as mean and precision parameters. Derivation of the associated distribution function $F_{X}$ is not trivial, though several unique derivations do exist (Chhikara \& Folks, 1989). Given such difficulties it is somewhat surprising that the IG distribution function can actually be expressed somewhat simply in terms of the standard normal CDF $\Phi$.

$$
F_{X}(x)=\Phi\left[\sqrt{\frac{\lambda}{x}}\left(\frac{x}{\nu}-1\right)\right]+e^{2 \lambda / \nu} \Phi\left[-\sqrt{\frac{\lambda}{x}}\left(\frac{x}{\nu}+1\right)\right] .
$$

While we do no include a derivation of $F_{X}$ the interested reader can easily verify the result by noting that the derivative of (2.1.2) is in fact equal to the IG density function (2.1.1).

The popularity of the mean-precision parameterization of the IG density is largely due to two distinct features. First, as a practical matter, it can be shown that (2.1.1) is a special case of the Generalized Linear Models framework (Nelder \& Wedderburn, 1972) and, as such, general methods for model fitting are readily available. Secondly, and more pertinent

to our current purposes, the mean-precision parameterization of the IG distribution greatly facilitates several important theoretical results. As an example, derivation of the Moment Generating Function (MGF) for the IG distribution is rendered trivial by noting that (2.1.1) 
constitutes a natural exponential family with

$$
\begin{aligned}
f(x \mid \nu, \lambda) & =h(x) \exp \{\boldsymbol{\eta}(\boldsymbol{\theta}) \cdot \mathbf{T}(x)-A(\boldsymbol{\eta})\}, \\
h(x) & =\frac{1}{\sqrt{2 \pi x^{3}}}, \\
\mathbf{T}(x) & =(x, 1 / x)^{\top}, \\
\boldsymbol{\eta}(\nu, \lambda) & =\left(-\frac{\lambda}{2 \nu^{2}},-\frac{\lambda}{2}\right)^{\top}, \\
A(\boldsymbol{\eta}) & =-2 \sqrt{\eta_{1} \eta_{2}}-\frac{1}{2} \ln \left(-2 \eta_{2}\right) .
\end{aligned}
$$

It is well established that the MGF for a natural exponential family is given by $M_{\mathbf{T}}(\mathbf{u})=$ $\exp (A(\boldsymbol{\eta}+\mathbf{u})-A(\boldsymbol{\eta}))$ and, as a consequence, the MGF and Characteristic Functions for the Inverse-Gaussian distribution are then given as:

$$
\begin{aligned}
& M_{X}(t)=\exp \left\{\frac{\lambda}{\nu}\left[1-\left(1-\frac{2 \nu^{2} t}{\lambda}\right)^{1 / 2}\right]\right\}, \\
& C_{X}(t)=\exp \left\{\frac{\lambda}{\nu}\left[1-\left(1-\frac{2 i \nu^{2} t}{\lambda}\right)^{1 / 2}\right]\right\} .
\end{aligned}
$$

The moment generating function will be useful to us in two ways. At the outset, the first several moments of the IG will be of interest. It was stated previous that the $\operatorname{IG}(\nu, \lambda)$ distribution is characterized by a mean parameter $\nu$ and a parameter $\lambda$ related to the precision. These identities are easily established from the first two central moments which, given $M_{x}(t)$, can be shown to equal:

$$
\begin{gathered}
E[X]=m_{1}=\nu, \\
E\left[X^{2}\right]=m_{2}=\nu^{2}+\frac{\nu^{3}}{\lambda} .
\end{gathered}
$$

That the variance of $X$ is given by $\operatorname{var}(X)=\nu^{3} / \lambda$ easily follows.

Our principal use for the moment generating function will actually come in the following section where we will use the MGF to prove that the time it takes for a Wiener process with drift to first hit a given boundary value follows an Inverse-Gaussian distribution. This interpretation of the IG distribution will form the conceptual framework within which we develop our proposed First Hitting Time models. 


\subsection{The Inverse-Gaussian Distribution as the First Hitting Time of a Wiener Process}

We currently aim to rigorously establish the connection, mentioned above, between the Wiener process with drift and the inverse-Gaussian distribution. We begin with several definitions:

Definition 2.2.1. A process $X(t)$ is called a Wiener process with drift $\mu$ and instantaneous variance $\sigma^{2}$ if and only if it satisfies the following properties:

1. $X(t)$ is a continuous stochastic process, i.e. $X(t)$ is continuous at each time point $t>0$ with probability 1.

2. $X(t)$ has independent increments, i.e. for any $0<t_{1}<t_{2}<t_{3},\left(X\left(t_{2}\right)-X\left(t_{1}\right)\right)$ and $\left(X\left(t_{3}\right)-X\left(t_{2}\right)\right)$ are independent.

3. $X(t)$ has normally distributed increments with mean and variance proportional to the length of the increments, i.e. $(X(t+\delta)-X(t)) \sim N\left(\delta \cdot \nu, \delta \cdot \sigma^{2}\right)$.

If we further assume that $X(t)$ has initial state $X(0)=x_{0}$, then basic properties of the normal distribution imply that the marginal probability density function for $X(t)$ is also normal, given by

$$
f\left(x \mid x_{0}, t\right)=\frac{1}{\sqrt{2 \pi\left(t \sigma^{2}\right)}} \exp \left(-\frac{\left(\left(x-x_{0}\right)-t \mu\right)^{2}}{2 t \sigma^{2}}\right) .
$$

Definition 2.2.2. $T$ is called the first hitting time of the process $X(t)$ to threshold $a$ if and only if $T=\inf \{t: X(t)=a\}$.

The infimum is used in the preceding definition to ensure that the first hitting time is well-defined in the case that $X(t)$ never reaches the threshold $a$, in which case $T=\infty$.

With the above definitions and the form of the IG moment generating function, we are in a position to prove that the first hitting time $T$ of a Wiener process $X(t)$ to a threshold $a<x_{0}$ has an inverse-Gaussian distribution. To do so we will derive an expression for the Laplace transform $\mathcal{L}_{T}$ of the probability density function for $T$, conditional on $T<\infty$, 
and then show that $\mathcal{L}_{T}$ is equal to the moment generating function (2.1.4) for the inverseGaussian distribution derived earlier.

Let $f\left(x \mid x_{0}, t\right)$ be as in (2.2.1) and let $g\left(t \mid x_{0}, a\right)$ be the probability density function of $T$ given $T<\infty$. Consider the following Laplace transforms:

$$
\begin{aligned}
& f^{*}\left(x \mid x_{0}, \theta\right)=\int_{0}^{\infty} e^{-\theta t} f\left(x \mid x_{0}, t\right) d t \\
& g^{*}\left(\theta \mid x_{0}, a\right)=\int_{0}^{\infty} e^{-\theta t} g\left(t \mid x_{0}, a\right) d t .
\end{aligned}
$$

Then the following theorem can be used to find $g^{*}$.

Theorem 2.2.1. If $x<a<x_{0}$, then

$$
P(T<\infty) \cdot g^{*}\left(\theta \mid x_{0}, a\right)=\frac{f^{*}\left(x \mid x_{0}, \theta\right)}{f^{*}(x \mid a, \theta)}
$$

Proof. Consider the case where, for times $0<s<t$ and function values $x<a<x_{0}$, the Wiener process $X$ proceeds from $X(0)=x_{0}$ and first reaches $a$ at time $s$ (i.e. $T=s$ ). Then, in time $t-s$ the process proceeds from $X(s)=a$ to $X(t)=x$. Under these conditions the density of the final state of the process $X$ at time $t$ can be expressed as the following convolution:

$$
f\left(x \mid x_{0}, t\right)=P(T<\infty) \int_{0}^{t} g\left(s \mid x_{0}, a\right) f(x \mid a, t-s) d s
$$

Applying the Laplace transform to both sides of equation (2.2.5) yields the following:

$$
\begin{aligned}
\mathcal{L}\left\{f\left(x \mid x_{0}, t\right)\right\} & =\mathcal{L}\left\{P(T<\infty) \int_{0}^{t} g\left(s \mid x_{0}, a\right) f(x \mid a, t-s) d s\right\} \\
& =P(T<\infty) \mathcal{L}\left\{\int_{0}^{t} g\left(s \mid x_{0}, a\right) f(x \mid a, t-s) d s\right\} \\
& =P(T<\infty) \mathcal{L}\left\{g\left(s \mid x_{0}, a\right)\right\} \mathcal{L}\{f(x \mid a, t-s) d s\} \\
& =P(T<\infty) g^{*}\left(\theta \mid x_{0}, a\right) f^{*}(x \mid a, \theta)
\end{aligned}
$$

The second equality is due to the linearity of the Laplace transform. The third equality is due to the Convolution Theorem which states that the Laplace transform of the convolution of two one-sided functions is the product of the Laplace transforms of the two functions. 
The result then follows immediately from (2.2.6).

In order to derive an expression for $g^{*}$ from Theorem 2.2.1 it is necessary to find an expression for $f^{*}\left(x \mid x_{0}, \theta\right)$. Fortunately the integration in Equation (2.2.2) can be solved without much difficulty. The exponential term in Equation (2.2.2) can be rewritten as

$$
\begin{aligned}
-\theta t-\frac{\left(\left(x-x_{0}\right)-t \mu\right)^{2}}{2 t \sigma^{2}} & =-\left(\theta t+\frac{\left(x-x_{0}\right)^{2}}{2 \sigma^{2} t}-\frac{\mu\left(x-x_{0}\right)}{\sigma^{2}}+\frac{\mu^{2} t}{2 \sigma^{2}}\right) \\
& =-\frac{c}{2 d^{2} t}(t-d)^{2}-\frac{x-x_{0}}{\sigma^{2}}\left(\sqrt{\mu^{2}+2 \sigma^{2} \theta}-\mu\right),
\end{aligned}
$$

where

$$
c=\frac{\left(x-x_{0}\right)^{2}}{\sigma^{2}} \text { and } d=\frac{x-x_{0}}{\sqrt{\mu^{2}+2 \sigma^{2} \theta}} .
$$

An expression for the Laplace transform $f^{*}$ can then easily be derived by restating it in terms of the first moment of the Inverse-Gaussian pdf:

$$
\begin{aligned}
f^{*}\left(x \mid x_{0}, \theta\right) & =\int_{0}^{\infty} \frac{1}{\sqrt{2 \pi\left(\sigma^{2}\right)}} \exp \left\{-\frac{c}{2 d^{2} t}(t-d)^{2}-\frac{x-x_{0}}{\sigma^{2}}\left(\sqrt{\mu^{2}+2 \sigma^{2} \theta}-\mu\right)\right\} d t \\
& =\frac{1}{\sigma \sqrt{c}} \exp \left\{\frac{x_{0}-x}{\sigma^{2}}\left(\sqrt{\mu^{2}+2 \sigma^{2} \theta}-\mu\right)\right\} \int_{0}^{\infty} t \sqrt{\frac{c}{2 \pi t^{3}}} \exp \left\{-\frac{c}{2 d^{2} t}(t-d)^{2}\right\} d t \\
& =\frac{d}{\sigma \sqrt{c}} \exp \left\{\frac{x_{0}-x}{\sigma^{2}}\left(\sqrt{\mu^{2}+2 \sigma^{2} \theta}-\mu\right)\right\}
\end{aligned}
$$

Theorem 2.2.1 then immediately implies the following expression:

$$
P(T<\infty) g^{*}\left(\theta \mid x_{0}, a\right)=\exp \left\{\frac{x_{0}-a}{\sigma^{2}}\left(\sqrt{\mu^{2}+2 \sigma^{2} \theta}-\mu\right)\right\}
$$

Equation (2.2.10) is used to establish the desired connection between the Wiener process and the IG distribution.

Theorem 2.2.2. Let $X(t)$ be a Wiener process with initial state $x_{0}$, negative drift $\mu<0$, and variance $\sigma^{2}$. Also, let $T$ be the first hitting time of $X$ to threshold $a<x_{0}$. Then

1. $P(T<\infty)=1$,

2. $T \sim I G(\nu, \lambda)$, with $\nu=\frac{a-x_{0}}{\mu}$ and $\lambda=\frac{\left(a-x_{0}\right)^{2}}{\sigma^{2}}$. 
Proof. (1) is easily demonstrated by noting that both $g^{*}$ and the RHS of (2.2.10) simplify to 1 when $\theta=0$. Therefore the equality reduces to $P(T<\infty)=1$ at $\theta=0$. Equation (2.2.10) can then be rewritten as

$$
\begin{aligned}
g^{*}\left(\theta \mid x_{0}, a\right) & =\exp \left\{\frac{\left(a-x_{0}\right) \mu}{\sigma^{2}}\left[1-\left(1+\frac{2 \sigma^{2} \theta}{\mu^{2}}\right)^{1 / 2}\right]\right\} \\
& =\exp \left\{\frac{\lambda}{\nu}\left[1-\left(1+\frac{2 \nu^{2} \theta}{\lambda}\right)^{1 / 2}\right]\right\} .
\end{aligned}
$$

We then note that the moment generating function for $T$ is given by $M_{T}(\theta)=E\left[e^{T \theta}\right]=$ $g^{*}\left(-\theta \mid x_{0}, a\right)$ which gives

$$
M_{T}(\theta)=\exp \left\{\frac{\lambda}{\nu}\left[1-\left(1-\frac{2 \nu^{2} \theta}{\lambda}\right)^{1 / 2}\right]\right\} .
$$

We recognize equation (2.2.12) as the moment generating function of an Inverse-Gaussian distribution $I G(\nu, \lambda)(2.1 .4)$, which establishes the result.

Theorem 2.2.3. Let $X(t)$ be a Wiener process with initial state $x_{0}$, positive drift $\mu>0$, and variance $\sigma^{2}$. Also, let $T$ be the first hitting time of $X$ to threshold $a<x_{0}$. Then

1. $P(T<\infty)=e^{2 \lambda / \nu}$,

2. $T \mid T<\infty \sim I G(-\nu, \lambda)$, with $\nu=\frac{a-x_{0}}{\mu}$ and $\lambda=\frac{\left(a-x_{0}\right)^{2}}{\sigma^{2}}$.

Proof. The proof is completely analogous to that for Theorem 2.2.2. With $\mu>0$, Equation (2.2.10) simplifies to

$$
\begin{aligned}
P(T<\infty) g^{*}\left(\theta \mid x_{0}, a\right) & =\exp \left\{\frac{\left(a-x_{0}\right) \mu}{\sigma^{2}}\left[1-\left(1+\frac{2 \sigma^{2} \theta}{\mu^{2}}\right)^{1 / 2}\right]\right\} \\
& =\exp \left\{\frac{\lambda}{\nu}\left[1+\left(1+\frac{2 \nu^{2} \theta}{\lambda}\right)^{1 / 2}\right]\right\} .
\end{aligned}
$$

Equation (2.2.13) simplifies to $P(T<\infty)=e^{2 \lambda / \nu}$, for $\theta=0$. Then the conditional moment-generating function of $T$ given $T<\infty$ is given as $M_{T \mid T<\infty}(\theta)=E\left[e^{T \theta} \mid T<\infty\right]=$ 
$g^{*}\left(-\theta \mid x_{0}, a\right)$ and has the form

$$
M_{T \mid T<\infty}(\theta)=\exp \left\{-\frac{\lambda}{\nu}\left[1-\left(1-\frac{2 \nu^{2} \theta}{\lambda}\right)^{1 / 2}\right]\right\} .
$$

We can recognize equation (2.2.15) as the moment-generating function for the inverseGaussian distribution $I G(-\nu, \lambda)$, again establishing the result.

Theorem 2.2.4. Let $X(t)$ be a Wiener process with initial state $x_{0}$, zero drift $\mu=0$, and variance $\sigma^{2}$. Also, let $T$ be the first hitting time of $X$ to threshold $a \neq x_{0}$. Then

1. $P(T<\infty)=1$,

2. $T$ has pdf $f(t)=\frac{\lambda^{1 / 2}}{\sqrt{2 \pi t^{3}}} \exp ^{-\lambda / 2 t}$, with $\lambda=\frac{\left(a-x_{0}\right)^{2}}{\sigma^{2}}$.

Proof. Following the proofs for the preceding two theorems, letting $\mu=0$ in (2.2.10) gives

$$
\begin{aligned}
P(T<\infty) g^{*}\left(\theta \mid x_{0}, a\right) & =\exp \left\{\frac{x_{0}-a}{\sigma^{2}} \sqrt{2 \sigma^{2} \theta}\right\} \\
& =\exp \{-\sqrt{2 \lambda \theta}\} .
\end{aligned}
$$

Setting $\theta=0$ in equation $(2.2 .16)$ once again establishes that $P(T<\infty)=1$. The expression for the moment-generating function of $T$ is again given by $M_{T \mid T<\infty}(\theta)=E\left[e^{T \theta} \mid T<\right.$ $\infty]=g^{*}\left(-\theta \mid x_{0}, a\right)$ which simplifies to

$$
M_{T \mid T<\infty}(\theta)=\exp \{-\sqrt{-2 \lambda \theta}\}
$$

Equation (2.2.18) is not actually the moment generating function for the Inverse-Gaussian distribution. It is possible to show that (2.2.18) is the MGF for a density function, closely related to the IG distribution, with the form

$$
f_{X}(x \mid \nu, \lambda)=\sqrt{\frac{\lambda}{2 \pi}} x^{-3 / 2} \exp \left(-\frac{\lambda}{2 x}\right) I(x>0) .
$$

We omit this exercise since the result is present in any number of standard texts on stochastic processes. Ross (2006), for example, proves the result using the common Reflection Principle approach. 


\subsection{First Hitting Time Models}

The previous two sections (2.1 and 2.2) were respectively concerned with the basic properties of the Inverse-Gaussian density and its genesis as the distribution of the random first hitting time $T$ of a Wiener process $X(t)$ to some threshold $a$. This relationship between the Wiener process and the Inverse-Gaussian distribution provides a useful conceptual framework when modeling time-to-event phenomena which are thought to arise as the result of some underlying development within a subject. Particularly, we will be interested in modeling the initial state $x_{0}$ and drift $\mu$ of the underlying Wiener process as these parameters are often clinically insightful.

As an example and illustrative metaphor of the value of modeling the underlying Wiener process, consider the conceptualization of death as the event which occurs when a subject's (nonnegative) health first reaches 0. It might be reasonable to assume that a subject's health behaves (at least approximately) as a Wiener process $X(t)$ over time: a subject's health process $X(t)$ starts at some initial value $x_{0}>0$, declines on average at a given rate $\mu$, but varies around the expected health value $x_{0}-\mu t$ until $X(t)$ first reaches 0 and the subject experiences death. If this is a reasonable description of subject specific health then we can model and test effects on initial health $\left(x_{0}\right)$ as well as long term health trends $(\mu)$. Recalling comments made in Chapter 1 , it will be of particular interest to understand how differences in initial health and health trend affect survival during different time periods. Discussion of these issues will be presented in the following section.

In accordance with the above metaphor, we use the phrase "First Hitting Time" (FHT) model) to describe any model which assumes that event times $T_{i}$ are the first hitting times to $a=0$ for an underlying latent Wiener health process $X_{i}(t)$ with initial health $x_{0}>0$, health drift $\mu$, and variance $\sigma^{2}$. From Theorems 2.2.2 to 2.2.4 it can be seen that, conditional on $T_{i}<\infty$, the distribution of $T_{i}$ is given by

$$
f\left(t \mid x_{0}, \mu, \sigma^{2}\right)=\frac{x_{0}}{\sqrt{2 \pi \sigma^{2} t^{3}}} \exp \left\{-\frac{\left(x_{0}+\mu t\right)^{2}}{2 \sigma^{2} t}\right\} I\{t>0\} .
$$

It should be noted that the density in (2.3.1) is dependent on $x_{0}, \mu$, and $\sigma$ only through 
the functions $x_{0} / \sigma$ and $\mu / \sigma$ and is thus over-parametrized. As a consequence, one of these three parameters can be set to a constant without loss of generality. Because the initial state $x_{0}$ and the drift $\mu$ are the more clinically interpretable parameters we choose to set $\sigma=1$. We then arrive at the following for the First Hitting Time density

$$
f\left(t \mid x_{0}, \mu\right)=\frac{x_{0}}{\sqrt{2 \pi t^{3}}} \exp \left\{-\frac{\left(x_{0}+\mu t\right)^{2}}{2 t}\right\} I\{t>0\}
$$

We refer to the density in (2.3.2) as the $\operatorname{IG}\left(x_{0}, \mu\right)$ density throughout the remainder of this work. From Equation (2.1.2) it can immediately be seen that the survival function for the FHT density is given by

$$
S\left(t \mid x_{0}, \mu\right)=1-F\left(t \mid x_{0}, \mu\right)=\Phi\left(\frac{\mu t+x_{0}}{\sqrt{t}}\right)-\exp \left\{-2 x_{0} \mu\right\} \Phi\left(\frac{\mu t-x_{0}}{\sqrt{t}}\right)
$$

where $\Phi(\cdot)$ is still the standard Normal CDF. Early examples of the the above $I G$ model applied to analysis of time-to-event data include Lancaster (1972), Eaton and Whitmore (1977), and G. Whitmore (1975).

In order to further develop an intuition about the FHT model parameters, we take the time to demonstrate two features of the FHT survival function which are conceptually satisfying. Given the previous development of the model, one might intuit that an increase in the initial state $x_{0}$, insofar as it moves the latent process $X(t)$ farther from absorption at $a=0$, should increase the survival function in a uniform manner. Similarly, an increase in the drift $\mu$ implies that a subject drifts more slowly toward the absorption barrier which, again, would seem to suggest a uniform increase in the survival function. It can, in fact, be shown that an increase in either parameter (holding the other constant) results in such a stochastic ordering. Consider, for example, the derivative of $S\left(t \mid x_{0}, \mu\right)$ with respect to $\mu$,

$$
\begin{aligned}
\frac{\partial S}{\partial \mu} & =\sqrt{t} \phi\left(\frac{\mu t+x_{0}}{\sqrt{t}}\right)+2 x_{0} \exp \left\{-2 x_{0} \mu\right\} \Phi\left(\frac{\mu t-x_{0}}{\sqrt{t}}\right)-\sqrt{t} \exp \left\{-2 x_{0} \mu\right\} \phi\left(\frac{\mu t-x_{0}}{\sqrt{t}}\right) \\
& =2 x_{0} \exp \left\{-2 x_{0} \mu\right\} \Phi\left(\frac{\mu t-x_{0}}{\sqrt{t}}\right),
\end{aligned}
$$

where $\phi(\cdot)$ is the standard normal density. The second equality holds because the first and third terms from the first line are in fact equal. The second line is positive for all $t>0$ 
due to $x_{0}$ being strictly positive. The above is sufficient to show that for $\mu_{2}>\mu_{1}$ and $T_{i} \sim f\left(t \mid x_{0}, \mu_{i}\right), S_{T_{2}}(t)>S_{T_{1}}(t)$ for all $t$. In other words, as long as the initial state $x_{0}$ is held constant, a strict ordering of drift terms $\mu_{i}$ results in an equivalent stochastic ordering of the event times $T_{i}$. A similar exercise can demonstrate an analogous result for the initial state parameter $x_{0}$.

As a second point of interest, the FHT model inherently includes the possibility of a "cure fraction". It can be seen from (2.3.3) that a positive drift (i.e. subjects on average get healthier) results in a survival function that does not converge to 0 :

$$
\mu>0 \Rightarrow \lim _{t \rightarrow \infty} S(t)=1-\exp \left\{-2 x_{0} \mu\right\}
$$

This ability to inherently model the possibility that some subjects will never experience the event of interest is particularly useful in scenarios such as cancer research where subjects may go into remission, never to experience disease progression.

Several other noteworthy features of the FHT model deserve explicit attention. The remainder of Chapter 2 is devoted to these various topics including discussion of the hazard function, likelihood construction, extensions to include covariate information, Bayesian modeling, and further comments on parameter interpretation.

\subsubsection{The Hazard Function}

In light of the prominent role that the hazard function has played historically in survival analysis, we now take the time to make several illustrative comments regarding the hazard function for the FHT model. From (2.3.2) and (2.3.3) it can be seen that the hazard function for the FHT model can be written as

$$
h\left(t \mid x_{0}, \mu\right)=\frac{f\left(t \mid x_{0}, \mu\right)}{S\left(t \mid x_{0}, \mu\right)}=\frac{\frac{x_{0}}{\sqrt{2 \pi t^{3}}} \exp \left\{-\frac{\left(x_{0}+\mu t\right)^{2}}{2 t}\right\}}{\Phi\left(\frac{\mu t+x_{0}}{\sqrt{t}}\right)-\exp \left\{-2 x_{0} \mu\right\} \Phi\left(\frac{\mu t-x_{0}}{\sqrt{t}}\right)} .
$$

Unfortunately neither the hazard function in (2.3.6) nor the corresponding hazard ratios are amenable to significant simplification in general, largely due to the dependence on the non-elementary Normal CDF. While this difficultly limits the general comments that can be 
made about the behavior of the hazard function, a few examples are sufficient to demonstrate the flexibility of the FHT model, especially where a proportional hazard assumption would be inappropriate. We adapt two examples from Aalen et al. (2008) which illustrate the effects of the initial state $x_{0}$ and the drift $\mu$, respectively, on the hazard function and hazard ratios.

Recall the discussion above concerning the effects of the initial state $x_{0}$ on the FHT survival function. It is intuitive to think of subjects with lower initial states as relatively more frail, i.e. susceptible to the event. Given this intuition we would expect the hazard function to increase with a decrease in the initial state $x_{0}$, at least initially. Such behavior can be seen in Figure 2.1. Of particular interest is the appearance of the larger hazard function "pushing up against" the y-axis. This is quite typical of FHT hazards when the initial state $x_{0}$ is small. Also worth noting is the appearance that the two hazard functions converge as $t$ increases. It can in fact be shown that the hazard function for two IG distributions that only differ in their initial health terms $x_{0}$ will always converge for $t \rightarrow \infty$. We interpret this phenomenon as highlighting that the effect of initial health on survival decreases as time increases. This is a prime example of proportional hazards violation which would render the Cox model inappropriate.

The effect of the drift parameter $\mu$ on the hazard function is quite different. It was shown previously that a decrease in the drift parameter $\mu$ results in a stochastic decrease in the event time $T$. We can understand this relationship intuitively by noting that when $\mu$ is made more negative, the "force" with which the health process $X_{i}(t)$ is "pushed" toward the absorption barrier is increased. This would lead one to expect decreases in $\mu$ to also be associated with an increased hazard, but an increased hazard which increases over time. As an example, imagine two "identical" healthy young adults, one of whom begins smoking where the other never does. We might conceptualize smoking as increasing the rate of health loss in those who take up that habit, even though its immediate effects are not necessarily substantial. At early stages we would expect little difference in the hazard for these two individuals because exposure has not had much time to "push" the smokers health down significantly. Over time, though, consistent smoking creates profound differences in the 


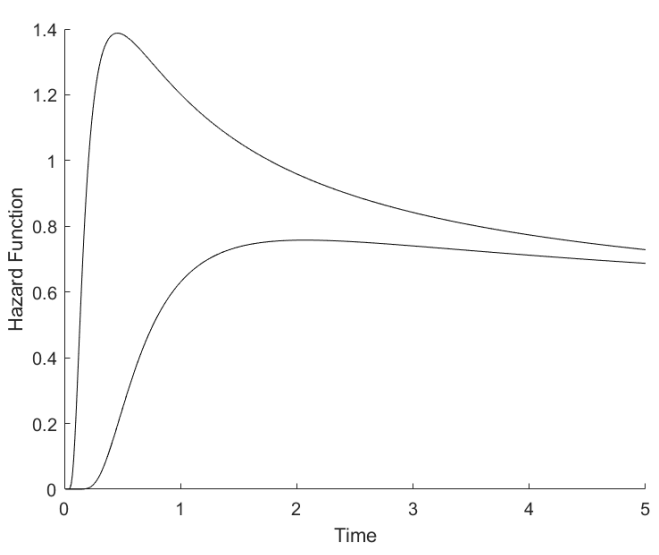

(a) Hazard Functions

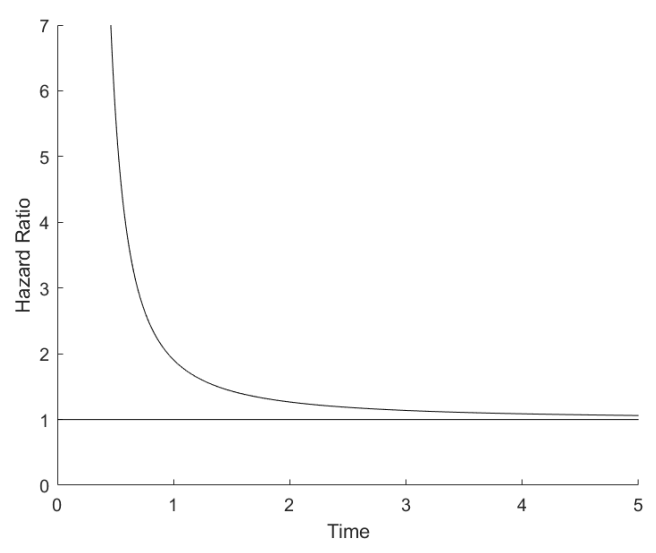

(b) Hazard Ratio

Figure 2.1: Left panel: the hazard functions for the First Hitting Time distribution with initial states $x_{0}=1$ (upper curve) and $x_{0}=2$ (lower curve). Right panel: the ratio of the two hazard functions. Other parameters: $\mu=-1$ and $\sigma=1$.

expected health of the smoker compared to the non-smoker. Such a pattern is visible in Figure 2.2 which displays hazard functions for IG distributions that only differ in the drift term $\mu$. What is somewhat less visible in this figure is the fact that hazard proportionality, though not convergence, also eventually sets in for the case of differing health drifts.

The convergence to proportionality visible in both Figure 2.1 and Figure 2.2 is a natural consequence of the quasi-stationary distribution for the Wiener process with an absorption barrier. Although details of the derivation are omitted here, Aalen et al. (2008) demonstrate that the distribution of the Wiener process $X(t)$ with an absorption barrier, conditional on $X(t)$ having not reached the barrier, converges to a gamma distribution that is not dependent on $x_{0}$, i.e.

$$
\gamma(x)=\lim _{t \rightarrow \infty} P[X(t)=x \mid X(t)>0 \forall t \in(0, t)]=\frac{\mu^{2}}{\sigma^{4}} x \exp \left\{\frac{-|\mu| x}{\sigma^{2}}\right\} .
$$

It is shown later (2.5.4) that initializing the Wiener process with random initial state $x_{0} \sim \mathrm{Ga}(k,|\mu|)$ results in a marginal event time $T$ which is $\operatorname{Ga}\left(k / 2, \mu^{2} / 2\right)$ distributed. Of interest currently is the implication that, for a Wiener process with $\sigma^{2}=1$ and $x_{0}$ distributed according to the quasi-stationary distribution, the marginal event time $T$ has an exponential distribution with rate $\lambda=\mu^{2} / 2$. This rate $\lambda$ is in fact the hazard function of the 


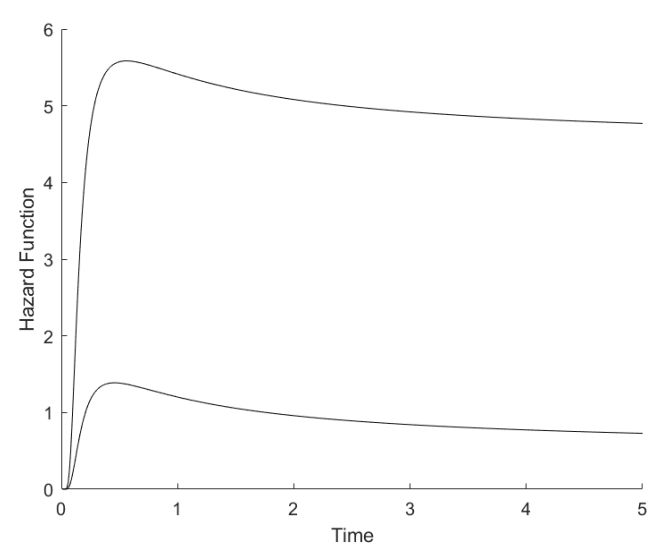

(a) Hazard Functions

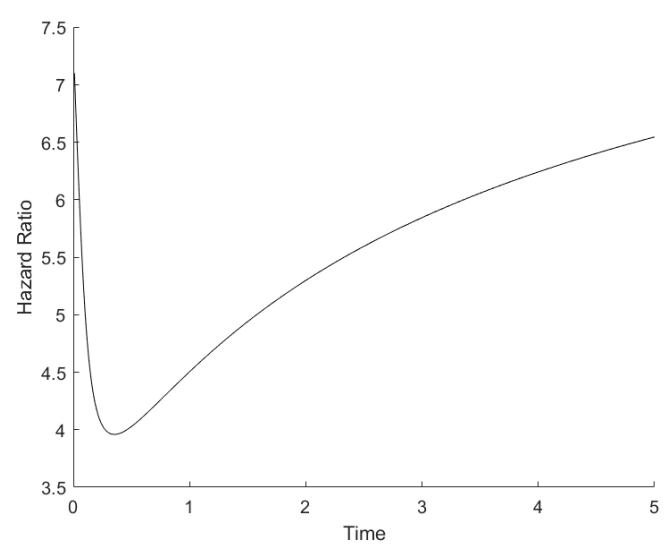

(b) Hazard Ratio

Figure 2.2: Left panel: the hazard functions for the First Hitting Time distribution with drifts $\mu=-3$ (upper curve) and $\mu=-1$ (lower curve). Right panel: the ratio of the two hazard functions. Other parameters: $x_{0}=1$ and $\sigma=1$.

exponential distribution. Further, the convergence of "surviving" Wiener processes to the quasi-stationary distribution $\gamma(x)$ implies that the hazard function for the FHT distribution must converge to the hazard function of the FHT model initialized with $x_{0}$ distributed according to the quasi-stationary distribution. This argument is sufficient to demonstrate that

$$
\lim _{t \rightarrow \infty} h(t)=\frac{1}{2} \mu^{2}
$$

independent of $x_{0}$, which explains the convergence behavior noted in Figure 2.1 and Figure 2.2.

\subsubsection{The Observed Data Likelihood}

Consider the general setting where data consist of observation times $T_{i}$ which are the minimum of the event times $S_{i}$ and censoring times $C_{i}$ (i.e. $T_{i}=\min \left\{S_{i}, C_{i}\right\}$ ). Event indicators $d_{i}$, which specify whether the event of interest was observed or censored (i.e. $\left.d_{i}=I\left\{S_{i}<C_{i}\right\}\right)$, are also recorded. For any fully parametric survival model with density function $f$ and survival function $S$, observed data likelihood functions can be constructed based on assumptions about the relationship between the censoring process and the data 
generation mechanism. For simplicity, survival times are often assumed independent of the censoring process which leads to the following observed data likelihood:

$$
L(\boldsymbol{\theta} \mid \mathbf{t}, \mathbf{d})=\prod_{i=1}^{n}\left[f\left(t_{i} \mid \boldsymbol{\theta}\right)\right]^{d_{i}}\left[S\left(t_{i} \mid \boldsymbol{\theta}\right)\right]^{1-d_{i}}=\prod_{i: d_{i}=1} f\left(t_{i} \mid \boldsymbol{\theta}\right) \prod_{i: d_{i}=0} S\left(t_{i} \mid \boldsymbol{\theta}\right) .
$$

Plugging the FHT density (2.3.2) and survival (2.3.3) functions into (2.3.9) will give the FHT likelihood which can be used in both ML procedures and Bayesian modeling. More comments regarding both possibilities will be made shortly.

Before continuing, it should be noted that a wide literature exists addressing cases where survival observations are inherently dependent and when the assumption of independent censoring is violated. Such considerations are beyond the scope of this work and their relation to the FHT model (and other models considered later) are left for future research.

\subsection{Threshold Regression Models}

Attention now turns to the inclusion of covariate information in the FHT model. Aalen and Gjessing (2001) suggest that one major appeal of the FHT model is the ability to distinguish between baseline $\left(x_{0}\right)$ and continuing $(\mu)$ covariate effects on survival. Stated in terms of the latent health process metaphor used previously, it is natural to think that covariates might variously affect a subject's initial health $\left(x_{0}\right)$ and/or trends in their health $(\mu)$. Given comments made in Section 2.3.1, we would interpret covariates associated with $x_{0}$ as those whose effects on survival decrease over time while covariates associated with $\mu$ are those whose effects grow more pronounced. One can model such covariate effects by relating regression structures to subject-specific parameters (i.e. $x_{0 i}$ and $\mu_{i}$ ) through specified link functions, analogous with Generalized Linear Models. Stated explicitly, one might specify the following model:

$$
\begin{aligned}
f\left(t_{i} \mid x_{0 i}, \mu_{i}\right) & =\frac{x_{0 i}}{\sqrt{2 \pi t_{i}^{3}}} \exp \left\{-\frac{\left(x_{0 i}+\mu_{i} t_{i}\right)^{2}}{2 t_{i}}\right\} I\left\{t_{i}>0\right\} \\
g\left(x_{0 i}\right) & =\mathbf{v}_{i}^{\top} \boldsymbol{\alpha} \\
h\left(\mu_{i}\right) & =\mathbf{w}_{i}^{\top} \boldsymbol{\beta}
\end{aligned}
$$


where $\mathbf{v}_{i}=\left(1, v_{i 1}, \ldots v_{i p}\right)^{\prime}$ and $\mathbf{w}_{i}=\left(1, w_{i 1}, \ldots w_{i q}\right)^{\prime}$ are covariate vectors for subject $i$ and $\boldsymbol{\alpha}=\left(\alpha_{0}, \ldots, \alpha_{p}\right)^{\prime}$ and $\boldsymbol{\beta}=\left(\beta_{0}, \ldots, \beta_{p}\right)^{\prime}$ are the corresponding coefficient vectors. While the choice of link functions $g(\cdot)$ and $h(\cdot)$ is usually context dependent, the model specification with a $\log$-link for $g(\cdot)$ and an identity-link for $h(\cdot)$ is a common choice as it restricts subjectspecific initial states to be positive and allows subject-specific drifts to take any value in $\mathbb{R}$. We refer to these link functions as the standard link functions and we refer the the resulting model as the standard Threshold Regression (TR) model. We denote this model using the following notation:

$$
\left.I G\left(\exp \left\{\mathbf{v}_{i}^{\top} \boldsymbol{\alpha}\right\}, \mathbf{w}_{i}^{\top} \boldsymbol{\beta}\right)\right)
$$

\subsubsection{Threshold Regression: ML Model Fitting}

It can be shown that maximization of the observed date likelihood $L(\boldsymbol{\alpha}, \boldsymbol{\beta} \mid \mathbf{t}, \mathbf{d})$ for the TR model is a nonlinear optimization problem, no matter the choice of link functions $g$ and

$h$. Consider the observed-data log-likelihood with arbitrary link-functions in the absence of censoring:

$$
l(\boldsymbol{\alpha}, \boldsymbol{\beta} \mid \mathbf{t}, \mathbf{d}) \propto \sum_{i=1}^{n} \log g^{-1}\left(\mathbf{v}_{i}^{\top} \boldsymbol{\alpha}\right)-\frac{\left(g^{-1}\left(\mathbf{v}_{i}^{\top} \boldsymbol{\alpha}\right)+h^{-1}\left(\mathbf{w}_{i}^{\top} \boldsymbol{\beta}\right) t_{i}\right)^{2}}{2 t_{i}} .
$$

Note that the first term in $(2.4 .3)$ can only be linear in $\boldsymbol{\alpha}$ if $g^{-1}(\cdot)=\exp (\cdot)$, in which case the second term is then a function of $\exp \left(\mathbf{v}_{i}^{\top} \boldsymbol{\alpha}\right)$, demonstrating nonlinearity. This lack of linearity is only exacerbated in the presence of censoring when the likelihood involves the normal CDF through the survival function.

Due to this nonlinearity, maximization of the TR log-likelihood function in practice generally involves numerical methods such as Newton-Raphson. Caroni (2017) reviews several software packages which have been developed for this purpose.

\subsubsection{Threshold Regression: Bayesian Modeling}

The Threshold Regression model extends to the Bayesian framework in standard fashion. By placing a prior distribution $\pi(\boldsymbol{\alpha}, \boldsymbol{\beta})$ on the regression parameters one derives, either 
analytically or numerically, the posterior distribution of model parameters according to Bayes' formula:

$$
\pi(\boldsymbol{\alpha}, \boldsymbol{\beta} \mid \mathbf{t}, \mathbf{d})=\frac{f(\mathbf{t}, \mathbf{d} \mid \boldsymbol{\alpha}, \boldsymbol{\beta}) \pi(\boldsymbol{\alpha}, \boldsymbol{\beta})}{f(\mathbf{t}, \mathbf{d})} \propto f(\mathbf{t}, \mathbf{d} \mid \boldsymbol{\alpha}, \boldsymbol{\beta}) \pi(\boldsymbol{\alpha}, \boldsymbol{\beta})
$$

Unfortunately, there are no known joint conjugate priors for the Bayesian Threshold Regression model except when (1) the regression equations on $x_{0}$ and $\mu$ are both "intercepts-only" structures, and (2) when there is no censoring. The conjugate prior exists in this case as a consequence of the density $f(t)$ belonging to an exponential family.

Given the lack of an analytical solution, Markov Chain Monte Carlo is commonly used to draw samples from the posterior distribution $\pi(\boldsymbol{\alpha}, \boldsymbol{\beta} \mid \mathbf{t}, \mathbf{d})$. The Metropolis-Hastings algorithm is particularly easy to implement and will be used for models developed later. Several papers, however, advocate the use of a unique Gibbs sampler (see Pennell, Whitmore, and Lee (2010), Lee, Shubina, and Zaslavsky (2005)). Such methods consider the state of the latent health process $X(t)$ at the time of censoring which has density

$$
f\left(X_{i}(t)=x_{i}, t_{i}<s_{i} \mid x_{0}, \mu\right)=\frac{1}{\sqrt{2 \pi t_{i}}} \exp \left\{-\frac{\left(x_{i}-\left(x_{0}+\mu t_{i}\right)\right)^{2}}{2 t_{i}}\right\}\left[1-\exp \left(-\frac{2 x_{i} x_{0}}{t_{i}}\right)\right],
$$

where the final state variables $x_{i}$ act as augmented parameters. Equation (2.4.5) then replaces $S\left(t_{i}\right)$ as the likelihood contribution for censored subjects. The benefit of this alternative sampling scheme is the existence of a normal conjugate prior for the conditional distribution of the drift regression coefficient $\boldsymbol{\beta}$. A Metropolis within Gibbs step is still necessary to sample the initial state regression term $\boldsymbol{\alpha}$. A simple Gibbs step also exists to sample from the augmented final state parameters $x_{i}$. Though analogous Gibbs sampling schemes are possible in all models developed in this work, we do not pursue such approaches for two main reasons: (1) ease of implementing the MH-sampler and (2) the ability for the MH-sampler to block sample both parametes $\boldsymbol{\alpha}$ and $\boldsymbol{\beta}$ which can be highly correlated.

Several applications of the Bayesian TR model exist in the literature. Upadhyay, Agrawal, and Smith (1996) and Balka, Desmond, and McNicholas (2011) apply models with regression terms on the drift $\mu$ only. Pettit and Young (1999) apply the Bayesian TR 
methodology to a case where degradation is observable for censored subjects. Pennell et al. (2010) propose a Bayesian Random Effects TR model, which we review in Section 2.5.4

\subsubsection{Threshold Regression: Collinearity}

It was noted earlier that one major appeal of the Threshold Regression model is its ability to distinguish covariate effects on the initial state and drift of the underlying Wiener process, at least theoretically. One might wonder, though, if such effects are actually identifiable when fitting the TR model to data with finite sample sizes. For example, consider the situation where both the initial state $x_{0}$ and drift $\mu$ increase linearly with a covariate $c_{i}$ and the "true" model is fit to data $\left(t_{i}, d_{i}, c_{i}\right), i \in\{1, \ldots, n\}$. Because an increase in the covariate is associated with an increase in survival through both $x_{0}$ and $\mu$, it is not intuitively obvious that some of the effect of $\alpha_{c}$ won't transfer to estimates for $\beta_{c}$ or vice versa. This and similar concerns about identifiability/collinearity have been expressed by several authors (see Eberly, Grambsch, and Connett (2001) and Lee and Whitmore (2006)). To address these collinearity questions we review a simulation study performed by Stogiannis and Caroni (2013) which was designed to further explore the issue.

Stogiannis and Caroni begin their simulation study by considering four basic scenarios where data come from the TR model with a single covariate $c_{i}$. These four cases are as follows:

1. the covariate affects only $x_{0}\left(\alpha_{1} \neq 0, \beta_{1}=0\right)$,

2. the covariate affects only $\mu\left(\alpha_{1}=0, \beta_{1} \neq 0\right)$,

3. the covariate affects both $x_{0}$ and $\mu\left(\alpha_{1} \neq 0, \beta_{1} \neq 0\right)$ with effects in the same direction,

4. the covariate affects both $x_{0}$ and $\mu\left(\alpha_{1} \neq 0, \beta_{1} \neq 0\right)$ with effects in the opposite direction.

For each scenario a large number of datasets with both moderate $(n=50)$ and large $(n=100)$ sample sizes were simulated. For each simulated dataset, the TR model was fit with the covariate $c_{i}$ associated with both $x_{0}$ and $\mu$. Patterns concerning the rejection of the hypotheses $H_{0 \alpha}: \alpha_{1}=0$ and $H_{0 \beta}: \beta_{1}=0$ were explored. The first major result showed 
that parameter estimates appeared approximately unbiased for all four cases. Additionally, the Likelihood Ratio and Wald tests for $H_{0 \beta}$ under scenario (1) and $H_{0 \alpha}$ under scenario (2) were close to the nominal $5 \%$ level, even when the sample size was small. This suggests that the inclusion of extraneous covariates does not adversely affect inference. Despite these positive results, some peculiarities were noted for scenarios (3) and (4). Of principal interest, it was found that under scenario (3) the TR model tended to reject $H_{0 \alpha}$ and $H_{0 \beta}$ simultaneously at a rate lower than expected if the two tests were independent. This suggests that the model concentrates similar effects onto either $x_{0}$ or $\mu$. The opposite pattern was found under scenario (4), where the simultaneous rejection rate of $H_{0 \alpha}$ and $H_{0 \beta}$ was higher than expected under an independence assumption, suggesting that the TR model tends to exaggerate opposing effects on $x_{0}$ and $\mu$. Together with the observation that parameter estimates are still approximately unbiased and significance tests are close to nominal, these patters suggests that the parameter estimates under data scenarios (3) and (4) are significantly correlated.

Stogiannis and Caroni then consider the case where one covariate $\left(c_{i}\right)$ affects the initial state $x_{0}$ and a second covariate $\left(d_{i}\right)$ affects the drift $\mu$, i.e. it is assumed that $\alpha_{1} \neq$ $0, \alpha_{2}=0, \beta_{1}=0$, and $\beta_{2} \neq 0$. For each simulated dataset, the full TR model is fit, i.e. both covariates are allowed to enter the model through both $x_{0}$ and $\mu$. From these simulations similar patterns emerge. First, as in the previous case, parameter estimates appear unbiased and significance tests remain close to the nominal $5 \%$ level. Despite this, there is still a tendency for a strong effect on one parameter $\left(x_{0}, \mu\right)$ to appear as an effect on the other. This pattern, in conjunction with the first observation, again strongly suggests that parameter estimates are potentially significantly correlated. Lastly, the authors note that as $\alpha_{1}$ increases, the power to reject $H_{0 \beta_{2}}: \beta_{2}=0$ also increases, and vice versa. The explanation for this phenomenon is that increases in $\alpha_{1}$ or $\beta_{2}$ imply a stochastic increase in event times. Longer event times make it easier to detect differences in $x_{0}$ and $\mu$. Stogiannis and Caroni summarize the results by noting that parameter estimation is quite well behaved. While there is some notable dependence between regression parameters $\boldsymbol{\alpha}$ and $\boldsymbol{\beta}$, there are no apparent biases in the estimates and sizes for the likelihood ratio and Wald tests are 
close to nominal across all cases considered.

\subsection{Heterogeneity in First Hitting Time Models}

We have noted at several points already that parametric models can suffer, sometimes severely, in the presence of unmeasured predictors or other sources of heterogeneity. Two popular methods which address such concerns have received significant attention in the broader statistics literature; random effects models and finite mixture models. Both of these methodologies have been applied in various ways to account for latent parameter heterogeneity in TR models. The current section reviews several of these efforts.

\subsubsection{Random Drift TR Model}

We first consider an extension of the Threshold Regression model (2.4.1) which includes a random intercept in the drift regression structure. This model has been given specific attention by Aalen et al. (2008). In order to construct the density for this model we begin by considering the standard FHT density (2.3.2) with initial state $x_{0}$ and subject-specific drift $\mu_{i}$. We assume that this subject-specific drift comes from a normal distribution with mean $m$ and variance $\tau^{2}$, which we label $\phi\left(\mu_{i} \mid m, \tau^{2}\right)$. In order to derive the marginal density of the event time, i.e. $f\left(t \mid x_{0}, m\right)$, it is necessary to integrate out the subject specific drift from the joint density $f\left(t \mid x_{0}, \mu_{i}\right) \phi\left(\mu_{i} \mid m, \tau^{2}\right)$, obtaining

$$
f_{R D}\left(t \mid x_{0}, m\right)=\int_{\mathbb{R}} f\left(t \mid x_{0}, \mu_{i}\right) \phi\left(\mu_{i} \mid m, \tau^{2}\right) d \mu_{i}=\frac{x_{0}}{\sqrt{2 \pi}} \frac{1}{t \sqrt{t^{2} \tau^{2}+t}} \exp \left\{-\frac{\left(x_{0}+m t\right)^{2}}{2\left(t^{2} \tau^{2}+t\right)}\right\} .
$$

Fortunately, the integration in (2.5.1) can be solved by a straightforward, though somewhat lengthy, manipulation of the quadratic forms in the exponents of the component densities. This exercise is omitted due to its length. The survival function corresponding to the density 
in (2.5.1) is given by:

$$
\begin{aligned}
S_{R D}\left(t \mid x_{0}, m\right) & =1-F_{R D}\left(t \mid x_{0}, m\right) \\
& =\Phi\left(\frac{m t+x_{0}}{\sqrt{t^{2} \tau^{2}+t}}\right)-\exp \left\{-2 x_{0} m+2 x_{0}^{2} \tau^{2}\right\} \Phi\left(\frac{m t-x_{0}-2 x_{0} t \tau^{2}}{\sqrt{t^{2} \tau^{2}+t}}\right) .
\end{aligned}
$$

As with the standard IG distribution, it is fairly simple to check that the density function in (2.5.1) is in fact the derivative of $F_{R D}$ defined in (2.5.2) which establishes the result. A more explicit derivation of the survival function in (2.5.2) can be found in Aalen et al. (2008, pg 409).

The density for the Randomized Drift FHT model given in (2.5.1) looks remarkably similar to the standard FHT density (2.3.2). In fact, one can easily see that the latter is a special case of the former with random effects variance $\tau=0$. It is important to emphasize, however, that parameter interpretation for $m$ in the Random Effect model differs slightly from that for $\mu$ in the standard model. Where a subject specific drift parameter $\mu_{i}$ would define the actual drift of subject $i$ 's latent Wiener process, $m_{i}$ merely defines the expected value of that drift. Fortunately, this slight distinction in parameter interpretation for $\mu$ and $m$ does not necessarily cause corresponding interpretation problems when the Randomized Drift FHT model is extended to incorporate covariate information. To see why consider relating covariates to model parameters $\left(x_{0 i}\right.$ and $\left.m_{i}\right)$ as in the standard TR model, i.e. allow

$$
\begin{aligned}
& g\left(x_{0 i}\right)=\mathbf{v}_{i}^{\top} \boldsymbol{\alpha}, \\
& h\left(m_{i}\right)=\mathbf{w}_{i}^{\top} \boldsymbol{\beta} .
\end{aligned}
$$

We refer to this model as the Randomized Drift Threshold Regression (RD-TR) model. For an arbitrary link-function $h$ the subject-specific drift can be written as $\mu_{i}=m_{i}+b_{i}=$ $h^{-1}\left(\mathbf{w}_{i}^{\top} \boldsymbol{\beta}\right)+b_{i}$ where $b_{i} \sim N\left(0, \tau^{2}\right)$. Due to the linearity of the normal distribution, when $h$ is chosen to be the identity-link $(h(x)=x)$ the random drift for subject $i$ can be simplified to $\mu_{i}=\mathbf{w}_{i}^{\top} \boldsymbol{\beta}+b_{i}=\left(\beta_{0}+b_{i}\right)+\sum_{j=1}^{q} w_{i j} \beta_{j}$. This demonstrates that under the RD-TR model, $\beta_{0}$ is properly interpreted as the expected value of subject specific drifts when $w_{i 1}=\ldots=$ 
$w_{i q}=0$. The parameters $\beta_{1}, \ldots \beta_{q}$, on the other hand, maintain their interpretation from the standard TR model as population regression coefficients and thus are directly relatable between the two models. Note, however, that this result is dependent on the assumption of the identity-link for $h$. For an arbitrary link function the subject specific drift would be expressed as $\mu_{i}=h^{-1}\left(\mathbf{w}_{i}^{\top} \boldsymbol{\beta}\right)+b_{i} \neq h^{-1}\left(\mathbf{w}_{i}^{\top} \boldsymbol{\beta}+b_{i}\right)$. As a consequence, the relatability of regression parameters between the standard and random effects models is not guaranteed for all choices of $h$. This result should not be surprising since an analogous phenomenon occurs in the broad case of Generalized Linear Models.

Aalen et al. (2008) present a data application to highlight the utility of the Random Drift TR model. The data for this application come from a study concerning treatment for carcinoma of the oropharynx. In addition to survival time from diagnosis (or censoring time), covariate information such as subject demographics and cancer state at time of diagnosis are available. Of particular interest is the fact that the fitted RD-TR model assigns the effects of cancer stage to the initial health parameter $x_{0}$. This outcome is conceptually pleasing as it conforms to the intuition that stage of cancer at baseline should be more closely related to baseline health than to health trends.

\subsubsection{Random Initial State FHT Model}

Aalen et al. (2008) also consider the possibility of mixtures over the random initial state in the FHT model. Specifically, they consider what would happen if subject specific values for the initial state $x_{0 i}$ were assumed to follow a gamma distribution proportional to

$x_{0}^{k-1} \exp \left(-|\mu| x_{0}\right)$ with $\mu<0$. Using moment generating functions it can then be shown 
that the marginal distribution of the event time $T$ is also gamma distributed:

$$
\begin{aligned}
M_{T}(\theta) & =E\left[e^{\theta T}\right]=\int e^{\theta t} h(t) \mathrm{d} t \\
& =\int e^{\theta t} \int f\left(t \mid x_{0}, \mu\right) g\left(x_{0}\right) \mathrm{d} x_{0} \mathrm{~d} t \\
& =\int g\left(x_{0}\right) \int e^{\theta t} f\left(t \mid x_{0}, \mu\right) \mathrm{d} t \mathrm{~d} x_{0} \\
& =\int \frac{|\mu|^{k}}{\Gamma(k)} x_{0}^{(k-1)} \exp \left\{-|\mu| x_{0}\right\} \cdot \exp \left\{x_{0}|\mu|\left[1-\left(1-\frac{2 \theta}{\mu^{2}}\right)^{1 / 2}\right]\right\} \mathrm{d} x_{0} \\
& =\frac{|\mu|^{k}}{\Gamma(k)} \int x_{0}^{(k-1)} \exp \left\{-x_{0}|\mu|\left(1-\frac{2 \theta}{\mu^{2}}\right)^{1 / 2}\right\} \mathrm{d} x_{0} \\
& =\frac{|\mu|^{k}}{\Gamma(k)} \cdot \frac{|\mu|^{k}\left(1-\frac{2 \theta}{\mu^{2}}\right)^{k / 2}}{\left.\mid 1-\frac{2}{\mu^{2}} \theta\right)^{-k / 2}} .
\end{aligned}
$$

The last line of (2.5.4) is the moment generating function for a $\mathrm{Ga}\left(k / 2, \mu^{2} / 2\right)$ distribution with density

$$
f(t \mid \mu, k)=\frac{\left(\mu^{2} / 2\right)^{k / 2}}{\Gamma(k / 2)} t^{k / 2-1} \exp \left\{-\frac{\mu^{2}}{2} t\right\} .
$$

There are several disadvantages of this proposed Random Initial State FHT model. The first and major disadvantage is the loss of the conceptual framework and accompanying interpretability from the FHT model. The density in (2.5.5) no longer depends on anything like the initial state term $x_{0}$. Instead, the parameter $k$ controls whether the underlying Wiener process is initialized with $x_{0}$ stochastically smaller $(0<k<1)$, stochastically larger $(k>1)$, or stochastically equal $(k=1)$ to the Quasi-stationary distribution. Admittedly, under this interpretation it is possible to link regression coefficients to the parameter $k$ in order to learn about effects on the distribution of subject-specific initial states $x_{0 i}$. Note, however, that interpretation of any regression coefficients on $k$ is not unambiguous, especially if regression coefficients are included on the drift $\mu$. Because the Wiener process initial state $x_{0 i}$ is initialized according to a mixture distribution dependent on the drift, allowing variability in the drift implies variability in the mixture distribution for $x_{0 i}$. 
The second major disadvantage of the Random Initial State FHT model is the resulting hazard function. It is well known that the hazard function for a Gamma random variable is monotone or constant. It is somewhat ironic, and patently undesirable, that the flexibility of the FHT hazard function is lost when a random initial state is included in order to allow for heterogeneity.

\subsubsection{Normal-gamma Mixtures of IG Distributions}

Although not directly relevant to the models developed later in this work, it is worth noting that modeling of heterogeneity in First Hitting Time models can be more easily facilitated by a reparameterization. Recall from (2.3.1) that the First Hitting Time density is overparameterized in terms of the three parameters defining the underlying Wiener process $\left(x_{0}, \mu, \sigma^{2}\right)$ and that one of these can be set to a constant without loss of generality. If one takes the alternative option of setting $x_{0}=1$ the resulting FHT density can be written as

$$
f\left(t \mid \mu, \sigma^{2}\right)=\left(\frac{\lambda}{2 \pi t^{3}}\right)^{1 / 2} \exp \left\{-\frac{\lambda(1+\mu t)^{2}}{2 t}\right\} I\{t>0\}
$$

where $\lambda$ is the reciprocal of the variance. While the density (2.5.6) is not used extensively in this work due to a lack of a clinically relevant interpretation for the variance term, it is worth mentioning that this parameterization is more amenable to mixture models than the standard FHT density given by (2.3.2). G. Whitmore (1986) develops a Normalgamma mixture of Inverse Gaussian distributions by assuming that the precision and drift parameters respectively have a gamma and normal distribution, i.e.

$$
\begin{aligned}
p(\lambda \mid a, b) & =\frac{b^{a}}{\Gamma(a)} \exp (-b \lambda), \\
p\left(\mu \mid \lambda, m, \tau^{2}\right) & =\frac{1}{\sqrt{2 \pi\left(\tau^{2} / \lambda\right)}} \exp \left\{-\frac{(\mu-m)^{2}}{2\left(\tau^{2} / \lambda\right)}\right\} .
\end{aligned}
$$

The resulting marginal event time density is then given by

$$
\begin{aligned}
f(t) & =\int_{0}^{\infty} \int_{-\infty}^{\infty} f\left(t \mid \mu, \sigma^{2}\right) p\left(\mu \mid \lambda, m, \tau^{2}\right) p(\lambda \mid a, b) d m d \lambda \\
& =\frac{\Gamma\left(a+\frac{1}{2}\right)}{\Gamma(a)}\left[2 \pi b t^{3}\left(t \tau^{2}+1\right)\right]^{-1 / 2}\left[1+\frac{(t m+1)^{2}}{2 b t\left(b \tau^{2}+1\right)}\right]^{-a-1 / 2} .
\end{aligned}
$$


As with the Randomized Drift TR model above, a regression model can then be placed on the mixture parameter $m$. In this case, regression coefficients are interpreted as effects on the expected value of the drift for the underlying subject specific Wiener process. While some flexibility is gained compared to the the RD-TR model with constant initial state $x_{0}$, the current mixture model lacks a clinically interpretable method of connecting covariate information to the non-drift term, i.e. to the precision parameter $\lambda$.

\subsubsection{Bayesian TR with Random Effects}

From the previous two sections it should be apparent that there are two principal difficulties in developing Threshold Regression models with random effects on both the initial state $x_{0}$ and drift $\mu$. The first of these difficulties is the derivation of a closed form for the marginal density. The second difficulty is an interpretable regression structure on the distribution of subject specific initial states $x_{0 i}$. Both of these problems, however, are readily addressed in the Bayesian framework where (a) Markov Chain Monte Carlo methods can overcome the inability to find a closed form for the marginal distribution and (b) regression structures involving random effects can be applied directly to the subject-specific parameters $x_{0 i}$ and $\mu_{i}$

Pennell et al. (2010), for example, develop a Bayesian Random Effects TR (BRE-TR) model which assumes a Normal random intercept in the drift function and positive-truncated Normal distribution on subject specific initial states. Their model has the following hierar- 
chical form:

$$
\begin{aligned}
f\left(t_{i} \mid x_{0 i}, \mu_{i}\right) & =\frac{x_{0 i}}{\sqrt{2 \pi t_{i}^{3}}} \exp \left\{-\frac{\left(x_{0 i}+\mu_{i} t_{i}\right)^{2}}{2 t}\right\} I\left\{t_{i}>0\right\} \\
\mu_{i} & =b_{i}+\mathbf{w}_{i}^{\top} \boldsymbol{\beta} \\
x_{0 i} & \sim N_{+}\left(\mathbf{v}_{i}^{\top} \boldsymbol{\alpha}, \tau^{-1}\right) \\
\boldsymbol{\beta} & \sim N\left(\boldsymbol{\beta}_{0}, \boldsymbol{\Sigma}_{\beta 0}\right) \\
b_{i} & \sim N\left(0, \lambda^{-1}\right) \\
\lambda & \sim G a\left(\gamma_{1}, \gamma_{2}\right) \\
\boldsymbol{\alpha} & \sim N\left(\boldsymbol{\alpha}_{0}, \boldsymbol{\Sigma}_{\alpha 0}\right)
\end{aligned}
$$

where $N_{+}(\cdot)$ represents the truncated Normal distribution on $[0, \infty)$. The choice of an identity-link for the initial state necessitates the specified truncated-Normal assumption on $x_{0 i}$ in order to guarantee all subject-specific initial states are strictly positive. Although it is a somewhat unconventional assumption, this identity-link facilitates posterior sampling through the existence of a conditional conjugate prior.

While further details of model construction and fitting for the BRE-TR model are not reviewed here, simulation study results published in the original paper are worth mentioning. The authors consider data simulated from (2.5.9) with parameters chosen to induce moderate heterogeneity:

$$
\begin{aligned}
& x_{0 i} \sim N_{+}\left(3-v_{i}, 1\right), \text { where } v_{i} \sim \operatorname{Bernoulli}(1 / 2), \\
& \mu_{i}=b_{i}+2.5-0.75 w_{i} \quad, \text { where } b_{i} \sim N(0,1) \text { and } w_{i} \sim N(4.5,0.36) .
\end{aligned}
$$

Of interest was the ability of a fitted BRE-TR model to accurately estimate the survival function at a variety of covariate combinations. The RD-TR model was also fit for the sake of comparison. Results of the study show that failure to account for the the specified heterogeneity in subject-specific initial state $x_{0 i}$ results in poor survival function estimation.

An extension of Pennell et al.'s BRE-TR model is also worth reviewing. Economou, 
Malefaki, and Caroni (2015) develop a generalization of (2.5.9) which can account for recurrent events. The generalization works roughly as follows. First we assume that subject $i$ experiences $n_{i}$ events which are ordered sequentially and occur at times $t_{1}, \ldots, t_{n_{i}}$. Further it is assumed that the time between events is $I G$ distributed, i.e. $s_{i m}=\left(t_{i m}-t_{i(m-1)}\right) \sim$ $I G\left(x_{0 i m}, \mu_{i m}\right)$. We call these $s_{i m}$ values "stages". Regression coefficients and random effects are added in a clever way. For the first stage, e.g. $s_{i 1}$, the regression structure comes directly from the Pennell model:

$$
\begin{aligned}
x_{0 i} & \sim N_{+}\left(\mathbf{v}_{i 1}^{\top} \boldsymbol{\alpha}, \tau^{-1}\right), \\
\mu_{i} & =b_{i 1}+\mathbf{w}_{i 1}^{\top} \boldsymbol{\beta} \text { with } b_{i 1} \sim N\left(0, \lambda^{-1}\right),
\end{aligned}
$$

where $\mathbf{v}_{i 1}$ and $\mathbf{w}_{i 1}$ are covariate values for the first event. For the remaining stages, the IG parameters $\left(x_{0 i m}, \mu_{i m}\right)$ are likely to be affected by both the total time that has passed and the number of events the subject has experienced so far. Additionally, it is possible that subject covariates change over time and it may be important to allow for such developments if they occur. Such effects can be captured by defining the IG parameters for subsequent events recursively as follows:

$$
\begin{gathered}
x_{0 i m} \sim N_{+}\left(\widetilde{\mathbf{v}}_{i m}^{\top} \boldsymbol{\alpha}^{*}, \tau^{-1}\right), \\
\mu_{i m}=\sum_{k=1}^{m} b_{i k}+\widetilde{\mathbf{w}}_{i m}^{\top} \boldsymbol{\beta}^{*},
\end{gathered}
$$

where

$$
\begin{aligned}
& \widetilde{\mathbf{v}}_{i m}^{\top} \boldsymbol{\alpha}^{*}=\mathbf{v}_{i 1}^{\top} \boldsymbol{\alpha}+\left(\mathbf{v}_{i m}^{\top}-\mathbf{v}_{i(m-1)}^{\top}\right) \boldsymbol{\alpha}_{d}+\alpha_{n s}(m-1)+\alpha_{t} t_{i(m-1)}, \\
& \widetilde{\mathbf{w}}_{i m}^{\top} \boldsymbol{\beta}^{*}=\mathbf{w}_{i 1}^{\top} \boldsymbol{\beta}+\left(\mathbf{w}_{i m}^{\top}-\mathbf{w}_{i(m-1)}^{\top}\right) \boldsymbol{\beta}_{d}+\beta_{n s}(m-1)+\beta_{t} t_{i(m-1)} .
\end{aligned}
$$

We reiterate that $b_{i m} \sim N\left(0, \lambda^{-1}\right)$ and $\left(\mathbf{v}_{i m}, \mathbf{w}_{i m}\right)$ are covariate values for stage $m$. We interpret these structures as follows:

1. $\boldsymbol{\alpha}$ and $\boldsymbol{\beta}$ are the effects of stage 1 covariates on the initial state and drift for the first event time, respectively.

2. $\boldsymbol{\alpha}_{d}$ and $\boldsymbol{\beta}_{d}$ are the effects of differences in covariates between adjacent stages. 
3. $\alpha_{n s}$ and $\beta_{n s}$ are the effects of an additional past event on the initial state and drift, respectively.

4. $\alpha_{t}$ and $\beta_{t}$ are the effects of total time passed on the initial state and drift, respectively.

Economou et al. provide details regarding model fitting, appropriate priors, simulation study results, etc. The methodology is applied to an interesting case study exploring patterns in treatment-seeking behavior among active drug users. Such information has significant public health interest as it can inform on best practices when it comes to intervention. The nature of drug use is such that relapse is common and several rounds of treatment may be necessary before a subject can completely break from substance abuse behaviors. Economou et al. apply their model to data concerning event times where an event is a subject seeking out treatment. Details of the results can be found in the published paper.

A final example of Bayesian TR with random effects comes from Saebo, Almoy, and Aastveit (2004) who use the TR paradigm to model time-to-disease in cattle. In animal husbandry it is often the case than a large population are the offspring of a limited number of sires who have desirable traits. The existence of a sire effect is likely to induce clustering of dependent subjects due to genetic similarity. It is usually beneficial to model such clustering by including random cluster effects. While Saebo et al. only allow for random sire effects on subject-specific drifts, their paper is noteworthy for highlighting the ability of Bayesian methods to accommodate a wide variety of structures in the TR paradigm.

\subsubsection{Threshold Regression: Finite Mixtures}

We now turn to a second method which has been used to account for latent parameter heterogeneity within Threshold Regression, namely, the use of finite mixtures. In order to develop a useful conception of their application to Threshold Regression, we will first establish the basic ideas behind finite mixture models in rather generic terms.

As a first step, consider a general specification of the random effects model:

$$
\begin{aligned}
X_{i} \stackrel{i n d}{\sim} F\left(\cdot \mid \theta_{i}, \psi\right), \\
\theta_{i} \sim G
\end{aligned}
$$


where (1) $\theta_{i}$ and $\psi$ are, respectively, subject-specific and population parameters for the data distribution $F$, and (2) $G$ is the modeled distribution for the subject-specific parameters. All of the random effects models in this chapter can be understood as special cases of (2.5.13). In practice, though, it will often be the case that we are uncomfortable with a strict assumption about the form of the mixture distribution $G$. Such cases would include the expectation that subject specific effects might be multi-modal or clustered. Clustering might occur, for example, in a clinical trial for a novel pharmaceutical whose effectiveness depends on subject genetics. If this dependence is such that the existence/absence of the relevant genes determines responders/nonresponders, then the resulting clustering of individuals would likely lead to a multi-modal distribution of subject specific treatment effects. Scenarios where similar latent heterogeneity exists in the data generation mechanism are remarkably easy to imagine and often difficult or impossible to avoid.

The basic idea of the finite mixture methodology is similar to the RE model in (2.5.13), with the important exception that a clustering assumption is made on the $\theta_{i}$ 's instead of a distributional assumption. Stated explicitly, the general finite mixture model can be expressed as follows:

$$
\begin{aligned}
& X_{i} \stackrel{i n d}{\sim} F\left(\cdot \mid \theta_{i}, \psi\right), i=1, \ldots, n, \\
& \theta_{i} \mid C_{i}=\theta_{C_{i}}^{*},
\end{aligned}
$$

where the set $\mathbf{C}=\left\{C_{1}, \ldots, C_{n}\right\} \in\{1, \ldots, K\}^{n}$ determines group/cluster membership for individual subjects (i.e. $\theta_{i}=\theta_{j} \Longleftrightarrow C_{i}=C_{j}$ ) and the set $\boldsymbol{\theta}^{*}=\left\{\theta_{1}^{*}, \ldots, \theta_{K}^{*}\right\}$ denotes the set of unique, group-specific parameters. $K$ specifies the number of clusters allowed by the model. Group membership is of course latent, analogous to the subject-specific parameters in the RE model. That the finite mixture model allows for increased model flexibility can also be understood by considering the population density which can be expressed as

$$
f\left(x \mid \boldsymbol{\theta}^{*}, \mathbf{p}\right)=\sum_{k=1}^{K} p_{k} f\left(x \mid \theta_{k}\right)^{*},
$$

where $p_{k}$ is the probability of being in cluster $k$. We mean here to indicate that the form of 
equation (2.5.15) allows the population density to potentially exhibit a plethora of distinct behaviors such as multimodality, severe skewness, thinner or thicker tails, etc.

While a complete review of the use of finite mixture models in the statistics literature will not be attempted here, it cannot be understated how important mixture models have been in the broader practice of statistics. A sufficient introduction can be found in Dempster, Laird, and Rubin (1977) who popularized the use of the EM algorithm to derive ML estimates under (2.5.15). The popular text from Gelman et al. (2013) offers a thorough treatment of finite mixture models in the Bayesian setting.

The finite mixture methodology has been applied to Threshold Regression in a number of ways. Given the preceding discussion, this application is fairly straightforward. The only additions to (2.5.14) that are needed to state the Finite Mixture for Threshold Regression (FM-TR) model are (1) inclusion of the IG distribution, (2) a regression structure, and (3) specification of which regression parameters are allowed to exhibit clustering behavior. As an example, the FM-TR model with clustering on all initial state and drift regression coefficients might be specified as

$$
\begin{gathered}
S_{i} \stackrel{i i d}{\sim} I G\left(\exp \left\{\mathbf{v}_{i}^{\top} \boldsymbol{\alpha}_{i}\right\}, \mathbf{w}_{i}^{\top} \boldsymbol{\beta}_{i}\right), \\
\boldsymbol{\alpha}_{i} \mid C_{i}=\boldsymbol{\alpha}_{C_{i}}^{*} \\
\boldsymbol{\beta}_{i} \mid C_{i}=\boldsymbol{\beta}_{C_{i}}^{*}
\end{gathered}
$$

Several notable papers have explored the use of models related to (2.5.16). A prime example comes from Lee, Chang, and Whitmore (2008) who use the finite mixture paradigm to account for dynamic treatment programs in a clinical trial of a novel myeloma drug. G. Whitmore and Su (2007) use finite mixtures to account for the possibility of abnormal fetal development in analyzing birth weight data.

\subsection{Summary}

The current chapter began with a derivation of the Inverse Gaussian distribution as the first-hitting time distribution for a Wiener process with drift. We then motivated and 
developed the First Hitting Time model as a useful and interpretable survival model based on this conception of the IG distribution. Lastly, we spent a significant amount of time reviewing several methods which have been explored to account for latent heterogeneity in the FHT model. One of these methods was the finite mixture model which, while providing flexible TR modeling, has the undesirable feature of requiring strict assumptions about the number of latent clusters which give rise to the data. The major contributions of the work will be the explorations of related TR models which do not require assumptions about the number of clusters. Before attempting to introduce these proposed models, though, it will be necessary to develop a generalization of the finite mixture model. This development will be the principal focus of the following chapter. 


\section{Chapter 3 \\ The Dirichlet Process Mixture}

My 20s were spent in a room, alone, mixing paints and figuring it all out.

- Caio Fonseca

The Dirichlet Process Mixture Model (DPMM) can be understood as a generalization of the finite mixture methodology introduced at the end of the previous chapter (2.5.14). One of the principal criticisms of the finite mixture is the somewhat arbitrary choice of the number of clusters $K$. While this parameter may occasionally be obvious for a given dataset due to preliminary analysis or the relevant science, this is in general the exception and not the rule. Additionally, it will often be the case that more groups become apparent as more data are included. It would be desirable, then, to develop mixture models where $K$ is not necessarily fixed and which are responsive to the complexity of the actual data at hand. The Dirichlet Process Mixture can be understood as a generalization of the finite mixture model which allows for just this type of flexibility. The present chapter is dedicated to developing the DPMM framework from this principal motivation.

\subsection{The Dirichlet Process Mixture Model}

Development of a working conception of the DPMM first requires a basic understanding of the Dirichlet distribution, commonly defined as follows:

Definition 3.1.1. A random variable $\mathbf{X}=\left(X_{1}, \ldots, X_{K}\right) \sim \operatorname{Dir}(\boldsymbol{\alpha})$ follows the Dirichlet distribution with parameter vector $\boldsymbol{\alpha} \in \mathbb{R}^{K}$ if its density is given by $f(\mathbf{x} \mid \boldsymbol{\alpha})=\frac{1}{D(\boldsymbol{\alpha})} \prod_{i=1}^{K} x_{i}^{\alpha_{i}-1}$, 
where

1. $\forall i x_{i} \in(0,1)$,

2. $\sum_{i} x_{i}=1$,

3. $D(\boldsymbol{\alpha})=\frac{\prod_{i=1}^{K} \Gamma\left(\alpha_{i}\right)}{\Gamma\left(\sum_{i=1}^{K} \alpha_{i}\right)}$.

The Dirichlet distribution is perhaps most familiar as the conjugate prior for the multinomial distribution. While this will not be our use, it highlights the fact that the Dirichlet distribution can be thought of as a "distribution over categorical distributions." Stated somewhat more technically, because of conditions (1) and (2) above, a sample $\mathbf{X}$ from the Dirichlet distribution is in fact a random categorical measure. In this sense the Dirichlet distribution is a distribution over all random discrete measures of dimension $K$.

The idea of random measures will play a key conceptual role in the current development of the DPMM. To motivate the direction our discussion will turn, consider again the general hierarchical model

$$
\begin{aligned}
X_{i} \stackrel{i n d}{\sim} F\left(\cdot \mid \theta_{i}, \psi\right), \\
\theta_{i} \sim G
\end{aligned}
$$

but assume that $G$ is a random discrete measure over $K$ potential, prespecified, parameter values $\boldsymbol{\theta}^{*}=\left(\theta_{1}^{*}, \ldots, \theta_{K}^{*}\right)$. Such a model would have limited usefulness because the choice of $\boldsymbol{\theta}^{*}$ would be quite arbitrary and, in fact, such a model is actually a restriction of the more general finite mixture model. Consider, however, the possibility of generalizing $\boldsymbol{\theta}^{*}$ to include countably infinite unique parameter values, "intelligently" spread throughout the domain of the subject specific parameters $\theta_{i}$. In this case $G$ would be a random measure controlling allocation of the finite samples to a finite subset of an infinite number of potential groups. The result would be a mixture model similar to (2.5.14) but without a set number of clusters $K$ from which the data must are drawn. The Dirichlet Process (DP), which we define below, essentially allows us to define distributions over infinite-dimensional random measures in the same way that the Dirichlet distribution allows us to define distributions over finite-dimensional random measures. 
Definition 3.1.2. Given a measurable set $\Theta$, a base probability distribution $H$, and a positive real number $\gamma$, a random distribution $G$ is distributed as a Dirichlet process $D P(\gamma H)$ if

$$
\left(G\left(B_{1}\right), \ldots, G\left(B_{r}\right)\right) \sim \operatorname{Dir}\left(\gamma H\left(B_{1}\right), \ldots, \gamma H\left(B_{r}\right)\right)
$$

for any finite measurable partition $B_{1}, \ldots, B_{r}$ of $\Theta$.

While, this formal definition of the Dirichlet Process does not immediately and obviously describe a distribution over random measures as suggested above, West, Mueller, and Escobar (1994) summarize the behavior of the DP in such a way that we can begin to illustrate these mechanisms. If $G \sim D P(\gamma H)$ and $\theta_{i} \mid G \stackrel{i i d}{\sim} G$ for $i=1, \ldots, n$, then

1. The values $\left\{\theta_{i}\right\}_{i=1}^{n}$ lie on a set of $K \leq n$ distinct values $\boldsymbol{\theta}^{*}=\left\{\theta_{1}^{*}, \ldots, \theta_{K}^{*}\right\}$.

2. The unique values $\theta_{j}^{*}$ are a random sample from $H$.

3. $K$ is random and is drawn from a distribution that is implicitly determined. $\mathrm{K}$ depends only on $n$ and $\gamma$ and increases stochastically with both. The distribution of $K$ is Poisson-like, though fatter tailed with asymptotic mean $\gamma \log (1+n / \gamma)$ for $n$ large.

4. Conditional on $K$, the values $\theta_{1}, \ldots, \theta_{n}$ are generated from the set $\left\{\theta_{1}^{*}, \ldots, \theta_{K}^{*}\right\}$ according to a uniform multinomial distribution.

The current point of interest is the distinct role played by $K$ in the DP compared to the finite mixture model. In the latter, $K$ is explicitly the number of allowed clusters. In the former, $K$ is a "dynamic" bound which adjusts for both the sample size $n$ and any apparent heterogeneity in the data. This dynamism is exactly the kind of behavior that we noted as desirable in motivating the Dirichlet Process.

Before proceeding to an introduction of the Dirichlet Process Mixture, it is insightful to understand the role played by the parameters in the DP. From (2) through (4) above it should be intuitive that $G$ is approximately equal to $H$ in distribution for large $\gamma$. It can be shown that $H$ is actually the expected value of $G$ and, as a consequence, is often called the base measure of the DP. Because $\gamma$ controls the variability of $G$ around $H$, with $G$ converging to $H$ as $\gamma$ increases, it is usually referred to as the precision parameter. This behavior can 

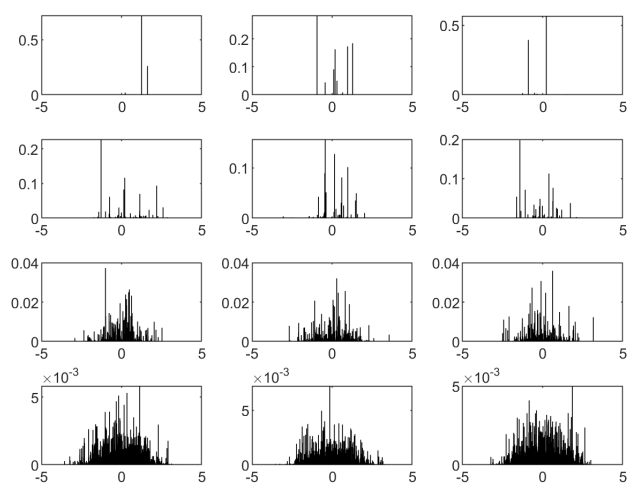

(a) Mass functions
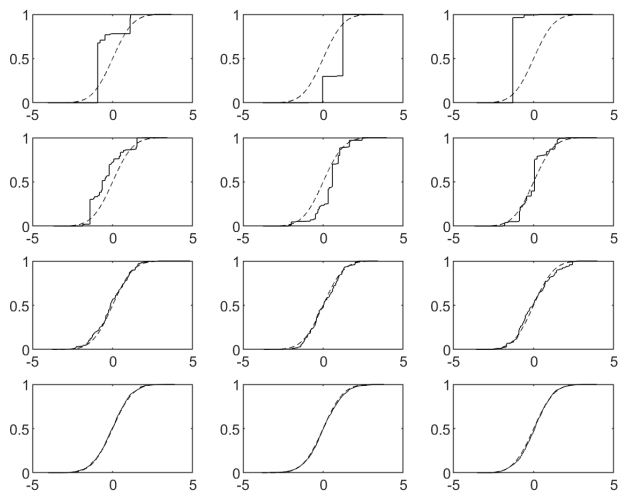

(b) Distribution functions

Figure 3.1: Three draws from the Dirichlet process for each value of the precision parameter $\gamma=1,10,100,1000$ (top to bottom). The mean parameter for all draws is $H \stackrel{D}{=} N(0,1)$. Panel (a): Random measures drawn from the Dirichlet Process. Panel (b): Distribution functions corresponding to the random measures in (a). The standard Normal CDF is included for reference.

be seen in Figure 3.1 which shows draws $G_{j}$ from the Dirichlet Process with base measure $H=N(0,1)$ and various values for the precision parameter $\gamma$. Figure 3.1 should drive home the fact that draws from the Dirichlet Process are in fact discrete random measures over an unbounded number of categories.

At this point it will be helpful to recall that we first motivated the finite mixture methodology by discussing a weakness of the general Random Effects model (2.5.13). We noted that it will often be the case that strong assumptions on the distribution $G$ of subject specific parameters $\theta_{1}, \ldots, \theta_{n}$ may be problematic. As an alternative, we might consider generalizing the Random Effect model by allowing the mixture distribution $G$ to be nonparametric, resulting in increased model flexibility. Such a specification can be accomplished by assuming that $G$ is in fact a draw from the Dirichlet Process. The resulting mixture model can be 
specified as follows:

$$
\begin{aligned}
\left(X_{i} \mid \theta_{i}, \psi\right) & \stackrel{i n d}{\sim} f\left(X_{i} \mid \theta_{i}, \psi\right), \\
\left(\theta_{i} \mid G\right) & \stackrel{i n d}{\sim} G, \\
\psi & \sim \pi(\psi), \\
G & \sim D P(\gamma H) .
\end{aligned}
$$

We call Model (3.1.2) the Dirichlet Process Mixture Model.

It is important to note that, as a practical matter, the model defined in (3.1.2) cannot readily be fit via likelihood methods. This difficulty relates to the fact that the Dirichlet Process does not have a likelihood that is easily written down. Fortunately, a variety of Markov Chain Monte Carlo methods exist which can facilitate model fitting in the Bayesian setting. In following sections we will review two distinct MCMC samplers for the DPMM which have received significant attention in the literature.

Given the necessarily Bayesian flair of the DPMM, further flexibility can be added to the model by allowing the precision parameter $\gamma$ to be random and by placing hyperpriors on the parameters controlling the base distribution $H$. Careful consideration of the prior on $\gamma$ is particularly important due to the relationship between $\gamma$ and the expected number of clusters under the DP. For example, it will often be the case that a relatively small number of clusters are sufficient to model any apparent latent heterogeneity. In such situations we might specify a prior for $\gamma$ which places more weight on smaller values, e.g. $\gamma \sim$ Gamma(1,1). Alternatively, specifying a prior which forces $\gamma$ to be extremely large will result in a model which approximates the Random Effects model with $H$ as the mixing distribution. The form for $H$ will often be chosen to facilitate computation and it will usually be desirable to specify vague hyperpriors on $H$. The set of hyperpriors controlling $\gamma$ and $H$ will be denoted $\boldsymbol{\eta}$ and a complete hierarchical DPMM can be specified by adding the line

$$
(\gamma, H \mid \boldsymbol{\eta}) \sim \pi(\gamma, H \mid \boldsymbol{\eta})
$$


to the model statement in (3.1.2).

Before continuing with our discussion of the DPMM, a few comments on terminology are in order. It has been emphasized repeatedly that draws from the Dirichlet process are themselves random measures or distributions. Throughout the remainder of the work we will have occasion to refer to both random measures drawn from the DP and samples generated from such random measures. To distinguish these two cases we adopt the following convention. We will call a random measure $G$ a draw from the $D P(\gamma H)$ if $G \sim D P(\gamma H)$. We will call the set $\boldsymbol{\theta}$ of random variables $\theta_{1}, \ldots \theta_{n}$ a sample from $D P(\gamma H)$ if

$$
\begin{aligned}
\theta_{i} \mid G & \stackrel{i n d}{\sim} G, \\
G & \sim D P(\gamma H) .
\end{aligned}
$$

This distinction between draws and samples from the DP should mitigate some bit of confusion when developing the Dirichlet Process Mixture Model further.

\subsection{DPMM - Polya-Urn Sampler}

The current section will review a Gibbs sampler for the DPMM which is based on the Polya urn interpretation of the DP. We note at the outset that we will occasionally omit some of the finer details in the interest of brevity. In most cases appropriate references will be included for the reader needing more depth. After introducing the Polya urn interpretation of the DP and the related Gibbs sampler, we will highlight several weaknesses which will prevent its use in our wider project. While we will forgo use of the Polya urn sampler for our developed methods, its inclusion here is justified on two counts. First, the Polya urn interpretation of the Dirichlet Process has played a historically significant role in the DPMM literature. As such, it seems necessary to justify why its use was not appropriate for our methods. Secondly, and more importantly, discussion of Polya urn sampler should assist the reader in developing more insight and understanding of the Dirichlet Process, which cannot hurt when we employ the DPMM methodology in later chapters. 


\subsubsection{Polya-urn Interpretation}

Consider a sample $\boldsymbol{\theta}=\left(\theta_{1}, \ldots, \theta_{n}\right)$ from $D P(\gamma H)$. Blackwell and MacQueen (1973) demonstrate that under the assumptions in (3.1.4), the following conditional prediction rule holds:

$$
\mathbf{P}\left(\theta_{i} \in \cdot \mid \boldsymbol{\theta}_{-i}\right)=\frac{\gamma}{\gamma+n-1} H(\cdot)+\frac{1}{\gamma+n-1} \sum_{j \neq i} \delta_{\theta_{j}}(\cdot),
$$

where $\boldsymbol{\theta}_{-i}$ represents the set $\left\{\boldsymbol{\theta} \backslash \theta_{i}\right\}$ and $\delta_{\theta_{j}}(\cdot)$ denotes the discrete measure concentrated at $\theta_{j}$. This prediction rule can be interpreted in terms of a modified Polya urn scheme which starts with $\gamma$ black balls and which develops as follows:

1. For each new observation $\theta_{i}$, draw a ball from the urn.

2. If the ball is black,

- Generate a new value $h \sim H$;

- Let $\theta_{i}=h$ and label a new white ball $h$. Place both the black and white ball in the urn.

3. If the ball is white and labeled $h$,

- Let $\theta_{i}=h$ and label a new white ball $h$. Place both the new and old white balls in the urn.

Not only does this Polya urn scheme result in a sample $\theta_{1}, \ldots, \theta_{n}$ from $D P(\gamma H)$ but also, as a consequence, the distribution $G$ over the values on the white balls in the urn converges to a random measure drawn from $D P(\gamma H)$ as $n \rightarrow \infty$. It should be noted, though, that the above interpretation requires that $\gamma$ be a positive integer. This restriction is not actually necessary for valid specification of the DP.

\subsubsection{The Polya Urn Gibbs Sampler}

Early work on the DPMM saw development of a Gibbs sampler based on the above Polya urn interpretation. The principal idea of this Gibbs sampler is to integrate over draws $G$ 
from the DP in (3.1.2) resulting in the marginalized hierarchical model given by

$$
\begin{aligned}
\left(X_{i} \mid \theta_{i}, \psi\right) & \stackrel{i n d}{\sim} f\left(X_{i} \mid \theta_{i}, \psi\right), \\
\boldsymbol{\theta} & \sim \pi(\boldsymbol{\theta}), \\
\psi & \sim \pi(\psi),
\end{aligned}
$$

where $\pi(\boldsymbol{\theta})$ denotes the joint distribution of the subject specific parameters under the Polya urn scheme. While the marginal distribution $\pi(\boldsymbol{\theta})$ does not in general have a closed form, an expression for the the conditional distribution $\pi\left(\theta_{i} \mid \boldsymbol{\theta}_{-i}, \psi, \gamma, \mathbf{X}\right)$ can be derived from the Polya urn prediction rule by making use of Bayes' theorem:

$$
\left(\theta_{i} \mid \boldsymbol{\theta}_{-i}, \psi, \gamma, \mathbf{X}\right) \sim q_{i} H_{i}+\sum_{j \neq i}^{n} q_{j} \delta_{\theta_{j}}
$$

where

1. $H_{i}$ is the posterior distribution of the observation $X_{i}$ under the baseline prior $H$, i.e. $H_{i} \propto f\left(X_{i} \mid \theta_{i}\right) d H\left(\theta_{i}\right)$.

2. The $q_{j}$ 's represent probabilities and must sum to 1 . The $q_{j}$ 's satisfy the following proportionality relationships:

$$
q_{j} \propto \begin{cases}\gamma \int_{\Theta} f\left(X_{i} \mid \theta, \psi\right) d H(\theta), & \text { if } j=i \\ f\left(X_{i} \mid \theta_{j}, \psi\right), & \text { else }\end{cases}
$$

(see Blackwell and MacQueen (1973), West et al. (1994), and Ishwaran and James (2001)). The form of the conditional posterior distribution $\pi(\psi \mid \boldsymbol{\theta}, \mathbf{X})$ can also be found using the standard application of Bayes' theorem:

$$
(\psi \mid \boldsymbol{\theta}, \mathbf{X}) \propto \pi(\psi) \prod_{i=1}^{n} f\left(X_{i} \mid \theta_{i}, \psi\right) .
$$

Recognizing the dependence of the conditional distributions above on $\gamma, H$, and $\pi$, it is clear that iteratively sampling from $(3.2 .3)$, for $i=1, \ldots, n$, and then from $(3.2 .5)$ is equivalent to a Gibbs sample from the conditional distribution $\pi(\boldsymbol{\theta}, \psi \mid \gamma, \boldsymbol{\eta}, \mathbf{X})$. A full Polya urn Gibbs step for the DPMM with hyperpriors is completed by sampling from 
the conditional posteriors for $\gamma$ and $\boldsymbol{\eta}$. It can, in fact, be shown that the assumption of independence between $\gamma$ and $\boldsymbol{\eta}$ a priori results in conditional independence between these parameters a posteriori as well (West, 1994). We derive an expression for the conditional distribution $\pi(\boldsymbol{\eta} \mid \boldsymbol{\theta}, \psi, \mathbf{X})$ presently.

Recall that under the DPMM (3.1.2) we have (1) hyperparameters $\boldsymbol{\eta}$ which enter the model only through $H$ and (2) conditional on $K$, the subject specific model parameters $\theta_{i}$ are a uniform multinomial sample from a set of unique parameters $\boldsymbol{\theta}^{*}=\left\{\theta_{1}^{*}, \ldots, \theta_{K}^{*}\right\}$ sampled from $H$. This implies that the conditional posterior density for the unique value $\theta_{j}^{*}$ can be expressed in terms of cluster membership $\mathbf{C}$, i.e.

$$
\pi\left(\theta_{j}^{*} \mid \psi, \mathbf{C}, \mathbf{X}\right) \propto d H\left(\theta_{j}^{*}\right) \prod_{\left\{i: C_{i}=j\right\}} f\left(X_{i} \mid \theta_{j}^{*}, \psi\right),
$$

where $C_{i}=j \Longleftrightarrow \theta_{i}=\theta_{j}^{*}$. From (1) it is also trivial to see that the posterior distribution on parameters controlling $H$ depends only on $\boldsymbol{\theta}^{*}$. Bayes' formula gives

$$
\pi\left(\boldsymbol{\eta} \mid \boldsymbol{\theta}^{*}, \psi, K, \mathbf{C}, \mathbf{X}\right) \equiv \pi\left(\boldsymbol{\eta} \mid \boldsymbol{\theta}^{*}\right) \propto \pi(\boldsymbol{\eta}) \prod_{j=1}^{K} d H\left(\theta_{j}^{*} \mid \boldsymbol{\eta}\right)
$$

The final step of the Polya urn sampler involves sampling from the posterior conditional distribution for the DP precision parameter $\gamma$. It is shown in West (1992) that the assumption of a $G a(a, b)$ prior on $\gamma$ results in the conditional posterior $\pi(\gamma \mid K)$ which can be sampled as follows:

1. Sample a value for the augmented variable $\alpha$ from the distribution $\alpha \sim \operatorname{Beta}\left(\gamma_{0}+1, n\right)$ where $\gamma_{0}$ is the current value for the DP precision parameter.

2. Sample the new value for the precision parameter $\gamma$ from the Gamma-mixture distribution

$$
(\gamma \mid x, K) \sim p_{x} \cdot G a(a+K, b-\log (\alpha))+\left(1-p_{x}\right) \cdot G a(a+K-1, b-\log (\alpha))
$$

where $p_{x}$ is defined by

$$
\frac{p_{x}}{1-p_{x}}=\frac{a+K-1}{n(b-\log (\alpha))}
$$


For details of the derivation the reader is directed to the published paper.

In summary, one full step under the basic Polya urn Gibbs sampler involves iteratively sampling from

1. $\left(\theta_{i} \mid \boldsymbol{\theta}_{-i}, \psi, \gamma, \mathbf{X}\right)$ for $i=1, \ldots, n$,

2. $(\psi \mid \boldsymbol{\theta}, \mathbf{X})$,

3. $\left(\boldsymbol{\eta} \mid \boldsymbol{\theta}^{*}, K\right)$,

4. $(\gamma \mid K)$,

all defined above.

\subsubsection{MCMC Mixing in the Polya Urn Sampler}

It is well established in the literature that the basic Polya urn sampling scheme described above has a tendency to mix slowly when the $q_{j}$ 's $(j \neq i)$ become significantly larger than $q_{i}$. The reason for slow mixing in this case is the resulting propensity of the sampler to get stuck at the current set of unique values $\boldsymbol{\theta}^{*}=\theta_{1}^{*}, \ldots, \theta_{m}^{*}$. A common method to facilitate mixing is to re-sample $\boldsymbol{\theta}^{*}$ conditional on cluster membership $\mathbf{C}$. This is accomplished by including a fifth step which samples from the condition distribution $\left(\theta_{j}^{*} \mid \psi, \mathbf{C}, \mathbf{X}\right)$ in equation (3.2.6). For further details and discussion on this method for improved mixing of the Polya urn Gibbs sampler, see West et al. (1994), Bush and MacEachern (1996), Escobar and West (1998) and Ishwaran and James (2001).

\subsubsection{Conjugacy in the Polya Urn Sampler}

The integral in equation (3.2.4) suggests that posterior computation for the Polya urn Gibbs sampler is greatly facilitated by $H$ being conjugate for $\theta_{i}$, in which case the integral is trivial. In the absence of conjugacy great care must be taken in computing the $q_{i}$ 's, though significant work has been done in this regard. West et al. (1994), for example, propose quadrature and Monte Carlo approximations for the $q_{i}$ 's. MacEachern and Mueller (1998) propose a Gibbs sampling step. Damlen, Wakefield, and Walker (1999) propose an auxiliary variable approach. Unfortunately, all of these solutions to the problem of non-conjugacy 
come at a computational cost.

This lack of a simple MCMC algorithm in the case of non-conjugacy is the principal reason that the Polya urn sampler will be undesirable in the current work. The preceding chapter discussed the reality that a full conjugate prior does not exist for TR where we wish to apply this methodology. As such, alternatives to the Polya urn sampler will be necessary. In Section 3.3 we will review just such an alternative sampling scheme which circumvents the above conjugacy problem without the need for post hoc numerical methods.

\subsection{DPMM - Blocked Gibbs Sampler}

The current section will develop the stick-breaking interpretation of the DP which allows for an arbitrarily precise approximation of the DPMM. These approximate models allow for an alternative sampling scheme which will more easily facilitate application of the DPMM methodology to TR.

\subsubsection{Stick-Breaking Interpretation}

As a contrast to the Polya urn illustration, the DP can be understood in terms of the socalled stick-breaking representation. It was noted earlier that the DP is a random measure which is discrete with probability 1 . The stick-breaking interpretation takes advantage of this fact and represents the DP as an infinite sum over point masses, i.e.

$$
\mathcal{G}_{\infty}(\cdot)=\sum_{k=1}^{\infty} p_{k} \delta_{\theta_{k}^{*}}(\cdot),
$$

where $\delta_{\theta_{k}^{*}}(\cdot)$ still denotes the discrete measure concentrated at $\theta_{k}^{*}$. This representation of the DP is controlled by a set of random masses $\left\{p_{k}\right\}_{k=1}^{\infty}$ over a set of random locations $\left\{\theta_{k}^{*}\right\}_{k=1}^{\infty}$. If the locations $\left\{\theta_{k}^{*}\right\}_{k=1}^{\infty}$ are drawn from $H$ and the masses $\left\{p_{k}\right\}_{k=1}^{\infty}$ are generated according to

$$
p_{k}=V_{k} \prod_{j=1}^{k-1}\left(1-V_{j}\right), \quad \text { with } V_{j} \stackrel{i i d}{\sim} \operatorname{Beta}(1, \gamma)
$$


then $\mathcal{G}_{\infty}$ is equivalent to the $D P(\gamma H)$ (Ishwaran and James, 2001). The name "stickbreaking" comes from the recognition that (3.3.2) is essentially a partitioning of the unit length interval. A DPMM can the be specified in a manner analogous to (3.1.2):

$$
\begin{aligned}
\left(X_{i} \mid \theta_{i}, \psi\right) & \stackrel{i n d}{\sim} f\left(X_{i} \mid \theta_{i}, \psi\right), \\
\left(\theta_{i} \mid G\right) & \stackrel{i n d}{\sim} G \\
\psi & \sim \pi(\psi), \\
G & \sim \mathcal{G}_{\infty} .
\end{aligned}
$$

\subsubsection{Truncation of the Stick-Breaking Representation}

As is the case with the Polya-urn scheme, the stick-breaking representation requires infinite information $\left(\left\{\theta_{k}^{*}\right\}_{k=1}^{\infty}\right.$ and $\left.\left\{p_{k}\right\}_{k=1}^{\infty}\right)$ to generate a random draw due to the infinite dimension of the Dirichlet Process. One benefit of the stick-breaking interpretation, however, is a natural finite-dimensional approximation. Consider the random measure generated by truncating the summation in (3.3.1) at $K$ locations, i.e.

$$
\mathcal{G}_{K}(\cdot)=\sum_{k=1}^{K} p_{k} \delta_{\theta_{k}^{*}}(\cdot),
$$

where the location and weight parameters are generated, analogously to (3.3.2), according to

$$
p_{k}= \begin{cases}V_{k} \prod_{j=1}^{k-1}\left(1-V_{j}\right), & \text { if } k \in\{1, \ldots, K-1\}, \\ 1-\sum_{k-1}^{K-1} p_{k}, & \text { if } k=K,\end{cases}
$$

with $V_{j} \stackrel{i i d}{\sim} \operatorname{Beta}(1, \gamma)$. Here, $p_{K}$ is assigned to be $p_{K}=1-\sum_{k=1}^{K-1} p_{k}$ to ensure that $\mathcal{G}_{K}$ is a valid measure. We refer to $\mathcal{G}_{K}$ with weights $\mathbf{p}$ defined by (3.3.5) as the Truncated Approximation (TA) measure.

The convergence of $\mathcal{G}_{K}(\cdot)$ to $\mathcal{G}_{\infty}(\cdot)$ as $K$ increases is trivial. Our current goal, however, is to establish that the mixture model (3.3.3) specified with the truncated stick-breaking prior $\mathcal{G}_{K}$ is a valid approximation to the DPMM specified with $\mathcal{G}_{\infty}$. It can be shown that the marginal distributions for a set of data under the two models can be made arbitrarily 
close by letting the truncation level $K$ go to infinity.

Theorem 3.3.1. Let $m_{\infty}(X)$ and $m_{K}(X)$ denote the marginal distributions of data $\mathbf{X}$ under the mixture model (3.3.3) with priors $\mathcal{G}_{\infty}$ and $\mathcal{G}_{K}$ respectively, i.e.

$$
\begin{aligned}
& m_{K}(\mathbf{X})=\int\left(\prod_{i=1}^{n} \int_{\mathcal{D}} f\left(X_{i} \mid \theta_{i}, \psi\right) \pi(\psi) d G\left(\theta_{i}\right)\right) d \mathcal{G}_{K}(G), \\
& m_{\infty}(\mathbf{X})=\int\left(\prod_{i=1}^{n} \int_{\mathcal{D}} f\left(X_{i} \mid \theta_{i}, \psi\right) \pi(\psi) d G\left(\theta_{i}\right)\right) d \mathcal{G}_{\infty}(G),
\end{aligned}
$$

where $\mathcal{D}$ is the domain of $\left[\psi, \theta_{i}\right]$. Also assume $H$ is nonatomic. Then the $\mathcal{L}_{1}$ distance between the two marginal distributions is bounded by

$$
\begin{aligned}
\left\|m_{K}(\mathbf{X})-m_{\infty}(\mathbf{X})\right\|_{1} & \leq 4\left[1-E\left\{\left(\sum_{k=1}^{K-1} p_{k}\right)^{n}\right\}\right] \\
& \approx 4 n \exp (-(K-1) / \gamma) .
\end{aligned}
$$

Proof. See Ishwaran and James (2001).

Theorem 3.3.1 tells us that the mixture model based on the truncated stick-breaking prior is actually an exponentially accurate approximation of the DPMM. While the term in the last line (3.3.7) offers an approximate bound on the $\mathcal{L}_{1}$ distance, the expectation in the first line can in fact be solved explicitly to give the following hard bound which can be useful in setting the truncation level.

\section{Corollary 3.3.1.1.}

$$
\left\|m_{K}(\mathbf{X})-m_{\infty}(\mathbf{X})\right\|_{1} \leq 4 \sum_{j=1}^{n}(-1)^{j+1}\left(\begin{array}{l}
n \\
j
\end{array}\right)\left(\frac{\gamma}{\gamma+j}\right)^{K-1} .
$$

Proof. We first note that the summation in (3.3.7) can be expressed in terms of a Gamma 
random variable.

$$
\begin{array}{rlr}
\sum_{k=1}^{N-1} p_{k} & =1-\left(1-V_{1}\right)\left(1-V_{2}\right) \ldots\left(1-V_{N-1}\right) & \\
& \stackrel{D}{=} 1-\exp \left(-\sum_{k=1}^{N_{1}} E_{k} / \gamma\right), & \\
& \stackrel{D}{=} 1-\exp \left(-\frac{1}{\gamma} \Gamma\right), & \text { where } E_{k} \stackrel{i i d}{\sim} \exp (1),
\end{array}
$$

The expectation in (3.3.7) can then be solved analytically.

$$
\begin{aligned}
E\left[\left(\sum_{k=1}^{N-1} p_{k}\right)^{n}\right] & =E\left[1-\exp \left(-\frac{1}{\gamma} \Gamma\right)\right]^{n} \\
& =\int(1-\exp \{-x / \gamma\})^{n} \frac{1}{\Gamma(N-1)} x^{(N-1)-1} e^{-x} d x \\
& =\int\left[\sum_{j=0}^{n}\left(\begin{array}{l}
n \\
j
\end{array}\right)\left(-e^{-x / \gamma}\right)^{j}\right] \frac{1}{\Gamma(N-1)} x^{(N-1)-1} e^{-x} d x \\
& =\sum_{j=0}^{n}(-1)^{j}\left(\begin{array}{l}
n \\
j
\end{array}\right) \int \frac{1}{\Gamma(N-1)} x^{(N-1)-1} e^{-(1+j / \gamma) x} d x \\
& =\sum_{j=0}^{n}(-1)^{j}\left(\begin{array}{l}
n \\
j
\end{array}\right) \frac{1}{(1+j / \gamma)^{N-1}} .
\end{aligned}
$$

The result then immediately follows.

\subsubsection{The Weak Limit Approximation of the DP}

A second approximation of the Dirichlet process has received significant attention in the literature and is worth reviewing. Ishwaran and Zarepour (2000, 2002) offer a thorough discussion of what we will call the Weak Limit Approximation of the DP. This approximation uses the same stick-breaking notation as in (3.3.4) with the exception that the $p_{k}$ 's are generated according to

$$
(\mathbf{p} \mid \gamma) \sim \operatorname{Dir}(\gamma / K, \ldots, \gamma / K)
$$

We use the notation $\mathcal{G}_{K_{W}}$ to denote the random measure (3.3.4) with $\mathbf{p}$ as in (3.3.9).

As was the case for the TA measure $\mathcal{G}_{K}$, the Weak Limit Approximation (WLA) measure 
$\mathcal{G}_{K_{W}}$ also turns out to be an asymptotically precise approximation of the Dirichlet Process. Ishwaran and Zarepour (2002) provide proof for the following relevant theorem.

Theorem 3.3.2. For all real valued measurable functions $f$ which are intergrable with respect to $H, \mathcal{G}_{K_{W}}(f) \stackrel{D}{\rightarrow} \mathcal{G}_{\infty}(f)$, as $K \rightarrow \infty$.

Proof. See Ishwaran and Zarepour (2002)

Given Theorem 3.3.2 and results related to the TA measure $\mathcal{G}_{K}$, it should come as little surprise that the mixture model (3.3.3) under the WLA measure $\mathcal{G}_{K_{W}}$ is also an arbitrarily precise approximation of the DPMM for large $K$. Analogous to Theorem 3.3.1, it is again possible to bound the $\mathcal{L}_{1}$ distance between marginal data distributions under mixture models with measures $\mathcal{G}_{\infty}$ and $\mathcal{G}_{K_{W}}$. The statement of this bound is facilitated by the following notation from Ishwaran and Zarepour (2002). Let $\mathbf{P}=\left\{C_{j}: j=1, \ldots, n(\mathbf{P})\right\}$ be a partition of the set $\{1, \ldots, n\}$ where $C_{j}$ is the $j$ th cluster of the partition, $e_{j}$ is the number of elements in cluster $C_{j}$, and $n(\mathbf{P})$ is the number of clusters in the partition. Additionally, for all $\gamma>0$ and $j \in \mathbb{N}^{+}$, let $\gamma^{[0]}=1$ and $\gamma^{[j]}=(\gamma+j-1) ! /(\gamma-1)$ !

Theorem 3.3.3. Let $m_{\infty}(\mathbf{X})$ and $m_{K_{W}}(\mathbf{X})$ denote the marginal distributions for data $\mathbf{X}$ under the mixture model (3.3.3) with mixture distributions $\mathcal{G}_{\infty}$ and $\mathcal{G}_{K_{W}}$ respectively, i.e.

$$
\begin{aligned}
m_{K_{W}}(\mathbf{X}) & =\int\left(\prod_{i=1}^{n} \int f\left(X_{i} \mid \theta_{i}, \psi\right) \pi(\psi) d G\left(\theta_{i}\right)\right) d \mathcal{G}_{K_{W}}(G), \\
m_{\infty}(\mathbf{X}) & =\int\left(\prod_{i=1}^{n} \int f\left(X_{i} \mid \theta_{i}, \psi\right) \pi(\psi) d G\left(\theta_{i}\right)\right) d \mathcal{G}_{\infty}(G) .
\end{aligned}
$$

Also assume $H$ is nonatomic. Then the $\mathcal{L}_{1}$ distance between the two marginal distributions is bounded by,

$$
\left\|m_{K_{W}}(\mathbf{X})-m_{\infty}(\mathbf{X})\right\|_{1} \leq \sum_{\mathbf{P}} \pi(\mathbf{P}) D(\mathbf{P})
$$

where

1. The sum is over all partitions $\mathbf{P}$ of $\{1, \ldots, n\}$.

2. $\pi(\mathbf{P})=\frac{\gamma^{k}}{\gamma^{(n)}} \prod_{j=1}^{k}\left(e_{j}-1\right)$ ! is the probability of a partition $\mathbf{P}$ under the $D P(\gamma H)$ prior. 
3. $D(\mathbf{P})= \begin{cases}\left\{\left|1-\frac{K !}{K^{k}(K-k) !}\right| \vee\left|1-n^{\gamma k / K}\right|\right\}, & \text { if } n(\mathbf{P}) \leq K, \\ 1, & \text { if } n(\mathbf{P})>K,\end{cases}$

where the variable $k=n(\mathbf{P}) \leq n$ represents the size of the partition and is used for clarity.

Proof. See Ishwaran and Zarepour (2002)

Unfortunately, the sum over all partitions in (3.3.11) makes it more difficult to utilize this bound in practice to establish an appropriate truncation level $K$. Despite this limitation, it is clear that for all $\mathbf{P}, D(\mathbf{P}) \rightarrow 0$ for $K \rightarrow \infty$. This implies that the given $\mathcal{L}_{1}$ distance also converges to 0 as $K$ increases, i.e. the mixture model based on the WLA measure $\mathcal{G}_{K_{W}}$ is also an arbitrarily precise approximation of the DPMM.

\subsubsection{Blocked Gibbs Sampler for Approximate DP Mixture Models}

The preceding sections demonstrate two key points regarding the Truncated Approximation measure $\mathcal{G}_{K}$ and the Weak Limit Approximation measure $\mathcal{G}_{K_{W}}:(1)$ both are arbitrarily precise approximations to the Dirichlet process $\mathcal{G}_{\infty}$, and (2) mixture models (3.3.3) with mixture distributions $\mathcal{G}_{K}$ and $\mathcal{G}_{K_{W}}$ are arbitrarily accurate approximations of the equivalent DPMM based on $\mathcal{G}_{\infty}$. We now take the time to establish a blocked Gibbs sampling scheme for mixture models based on these two approximating measures. Derivation of the blocked Gibbs sampler is greatly facilitated by restating the model (3.3.3) in terms of cluster membership C:

$$
\begin{aligned}
\left(X_{i} \mid \boldsymbol{\theta}^{*}, \mathbf{C}, \psi\right) \stackrel{\text { ind }}{\sim} f\left(X_{i} \mid \theta_{C_{i}}^{*}, \psi\right), \\
\left(C_{i} \mid \mathbf{p}\right) \sim \operatorname{Multinomial}(\mathbf{p}), \\
\left(\mathbf{p}, \boldsymbol{\theta}^{*}\right) \\
\sim \pi(\mathbf{p} \mid \gamma) \times d H^{K}\left(\boldsymbol{\theta}^{*}\right), \\
\psi \sim \pi(\psi),
\end{aligned}
$$

where $\mathbf{C}$ is again the set of latent clustering variables and $d H^{K}\left(\boldsymbol{\theta}^{*}\right)=\prod_{k}^{K} d H\left(\theta_{k}^{*}\right)$. The prior $\pi(\mathbf{p} \mid \gamma)$ is determined by the approximating measure chosen $\left(\mathcal{G}_{K}\right.$ or $\left.\mathcal{G}_{K_{W}}\right)$. We seek 
to derive a MCMC algorithm which can sample from the joint posterior distribution of all model parameters given the data, i.e. $\pi\left(\boldsymbol{\theta}^{*}, \mathbf{C}, \psi, \boldsymbol{\eta}, \mathbf{p}, \gamma \mid \mathbf{X}\right)$.

Using the model restatement in (3.3.12), a method for drawing a single Gibbs sample from the conditional distribution $\pi\left(\boldsymbol{\theta}^{*}, \mathbf{C}, \psi, \boldsymbol{\eta} \mid \mathbf{p}, \gamma, \mathbf{X}\right)$ becomes straightforward and can be broken down into the following steps.

1. Sample cluster-specific parameters, conditional on cluster membership. Recalling that subjects are independent conditional on their subject specific parameter values, i.e. $\left(X_{i} \mid \boldsymbol{\theta}^{*}, \mathbf{C}, \psi\right) \stackrel{i n d}{\sim} f\left(X_{i} \mid \theta_{C_{i}}^{*}, \psi\right)$, the following expression easily follows

$$
\pi\left(\theta_{k}^{*} \mid \mathbf{C}, \psi, \mathbf{X}\right) \propto d H(\boldsymbol{\theta}) \prod_{i=1}^{n} f\left(X_{i} \mid \boldsymbol{\theta}^{*}, \mathbf{C}, \psi\right) \propto d H\left(\theta_{k}^{*}\right) \prod_{\left\{i: C_{i}=k\right\}} f\left(X_{i} \mid \theta_{k}^{*}, \psi\right) .
$$

Note that in the case that cluster $j$ is empty (i.e. $\left\{i: C_{i}=k\right\}=\varnothing$ ) the likelihood contribution of the data is 1 and $\left(\theta_{k}^{*} \mid \mathbf{C}, \psi, \mathbf{X}\right) \sim H$. Iteratively sample from (3.3.13) for $k=1, \ldots, K$.

2. Sample new values for the shared parameters $\psi$. This is equivalent to the Polya urn sampler:

$$
\left(\psi \mid \boldsymbol{\theta}^{*}, \mathbf{C}, \mathbf{X}\right) \propto \pi(\psi) \prod_{i=1}^{n} f\left(X_{i} \mid \theta_{C_{i}}^{*}, \psi\right) .
$$

3. Sample new values for cluster membership conditional on cluster specific parameters $\boldsymbol{\theta}^{*}$ and stick-breaking weights $\mathbf{p}$. Sample from

$$
\pi\left(C_{i} \mid \boldsymbol{\theta}^{*}, \mathbf{p}, \psi, \mathbf{X}\right) \stackrel{i n d}{\sim} \sum_{k=1}^{K} p_{k, i} \delta_{k}(\cdot)
$$

for each subject $i=1, \ldots, n$, where $\left(p_{1, i}, \ldots, p_{K, i}\right) \propto\left(p_{1} f\left(X_{i} \mid \theta_{1}^{*}, \psi\right), \ldots, p_{K} f\left(X_{i} \mid \theta_{K}^{*}, \psi\right)\right)$.

4. Sample new values for the hyperparameters controlling the baseline prior $H$. This step is a direct application of Bayes' theorem because we have a sample $\boldsymbol{\theta}^{*}$ from $H$.

$$
\pi\left(\boldsymbol{\eta} \mid \boldsymbol{\theta}^{*}, \psi, K, \mathbf{C}, \mathbf{X}\right) \equiv \pi\left(\boldsymbol{\eta} \mid \boldsymbol{\theta}^{*}, K\right) \propto \pi(\boldsymbol{\eta}) \prod_{k=1}^{K} d H\left(\theta_{k}^{*} \mid \boldsymbol{\eta}\right)
$$

As stated earlier, the preceding steps constitute a posterior sample from $\pi\left(\boldsymbol{\theta}^{*}, \mathbf{C}, \psi, \boldsymbol{\eta} \mid \mathbf{p}, \gamma, \mathbf{X}\right)$. To complete a single step for the blocked sampler we must draw 
samples from $\pi\left(\mathbf{p}, \gamma \mid \boldsymbol{\theta}^{*}, \mathbf{C}, \psi, \boldsymbol{\eta}, \mathbf{X}\right)$. The method for doing so will depend on the prior choice for $\mathbf{p}$.

We first consider the case where the conditional distribution for the stick-breaking weights is assumed, a priori, to come from $(\mathbf{p} \mid \gamma) \sim \operatorname{Dir}(\gamma / K, \ldots, \gamma / K)$, i.e. the case where the Weak Limit Approximation measure $\mathcal{G}_{K_{W}}$ was chosen. In this case the following two steps will form a sample from the desired conditional distribution:

5. Sample new values for the random weights $\mathbf{p}$ conditional on the precision parameter $\gamma$. It is simple to see from (3.3.12) that this distribution depends only on the prior for $\mathbf{p}$ and the clustering variables $\mathbf{C}$ :

$$
\pi(\mathbf{p} \mid \gamma, \mathbf{C}) \propto f(\mathbf{C} \mid \mathbf{p}) \pi(\mathbf{p} \mid \gamma) \propto \prod_{k=1}^{K} p_{k}^{\gamma / K+m_{k}},
$$

where $m_{k}=\left|\left\{i: C_{i}=k\right\}\right|$ records the number of subjects assigned to each group $k$. This implies that the posterior for $\mathbf{p}$ is still Dirichlet distributed $(\mathbf{p} \mid \gamma, \mathbf{C}) \sim \operatorname{Dir}(\gamma / K+$ $\left.m_{1}, \ldots, \gamma / K+m_{K}\right)$.

6. Sample a new value for the DP precision parameter $\gamma$. Given the vector $\mathbf{p}$, Bayes' theorem immediately gives

$$
\pi(\gamma \mid \mathbf{p}) \propto \pi(\mathbf{p} \mid \gamma) \pi(\gamma) \propto\left(\frac{\Gamma(\gamma)}{\Gamma(\gamma / K)^{K}} \prod_{k=1}^{K} p_{k}^{\gamma / K-1}\right) \pi(\gamma)
$$

This form for $\pi(\gamma \mid \mathbf{p})$ is not a standard distribution, but can be sampled using a Random Walk Metropolis (RWM) step.

We also consider the case where $p_{k}=V_{k} \prod_{j=1}^{k-1}\left(1-V_{j}\right)$ with $V_{j} \stackrel{i i d}{\sim} \operatorname{Beta}(1, \gamma)$, i.e. the case where the TA measure $\mathcal{G}_{K}$ is chosen. To derive expression for the posterior conditional distributions $\pi(\mathbf{p} \mid \gamma, \cdot)$ and $\pi(\gamma \mid \mathbf{p})$ it will be necessary to appeal to the Generalized Dirichlet distribution.

Definition 3.3.1. A random variable $\mathbf{P}=\left(P_{1}, \ldots, P_{K}\right) \sim G D(\mathbf{a}, \mathbf{b})$ follows the Generalized 
Dirichlet distribution with parameter vectors $\mathbf{a}, \mathbf{b} \in \mathbb{R}^{K-1}$ if its density is given by

$$
\begin{aligned}
f(\mathbf{p} \mid \mathbf{a}, \mathbf{b})= & \left(\prod_{k=1}^{K-1} \frac{\Gamma\left(a_{k}+b_{k}\right)}{\Gamma\left(a_{k}\right) \Gamma\left(b_{k}\right)}\right) p_{1}^{a_{1}-1} \ldots p_{K-1}^{a_{K-1}-1} p_{K}^{b_{K-1}-1} \\
& \times\left(1-p_{1}^{*}\right)^{b_{1}-\left(a_{2}+b_{2}\right)} \ldots\left(1-p_{K-2}^{*}\right)^{b_{K-2}-\left(a_{K-1}+b_{K-1}\right)}
\end{aligned}
$$

where

1. $p_{k}^{*}=\sum_{j=1}^{k} p_{j}$,

2. $\forall i p_{i} \in(0,1)$,

3. $\sum_{i} p_{i}=1$.

The utility of the $G D$ distribution depends on the following theorem from Ishwaran and James (2002)

Theorem 3.3.4. Given parameter vectors $\mathbf{a}, \mathbf{b} \in \mathbb{R}^{K-1}$, let the vector of random variables $\mathbf{p} \in \mathbb{R}^{K}$ be generated by

$$
p_{k}=V_{k} \prod_{j=1}^{k-1}\left(1-V_{j}\right) \text { with } V_{j} \stackrel{i n d}{\sim} \operatorname{Beta}\left(a_{k}, b_{k}\right),
$$

with $p_{K}=1-\sum_{k=1}^{K-1} p_{k}$. Then $\mathbf{p} \sim G D(\mathbf{a}, \mathbf{b})$.

Theorem 3.3.4 implies that the random weights $\mathbf{p}$ under the TA measure $\mathcal{G}_{K}$ are $G D$ distributed. It is trivial to see that the $G D$ displays an analogous conjugacy with categorical variables as the Dirichlet distribution. The following steps to sample from $\pi\left(\mathbf{p}, \gamma \mid \boldsymbol{\theta}^{*}, \mathbf{C}, \psi, \boldsymbol{\eta}, \mathbf{X}\right)$ under the TA measure $\mathcal{G}_{K}$ are then easily derived.

5. Sample new values for the random weights $\mathbf{p}$. Given the preceding comments it can be seen that

$$
\pi(\mathbf{p} \mid \gamma, \mathbf{C}) \propto f(\mathbf{C} \mid \mathbf{p}) \pi(\mathbf{p} \mid \gamma) \propto G D\left(\mathbf{1}_{K-1}+\mathbf{m}, \gamma \cdot \mathbf{1}_{K-1}+\mathbf{M}\right)
$$

where $m_{k}=\left|\left\{i: C_{i}=k\right\}\right|$ still records the number of subjects assigned to each group $k$ and $M_{k}=\sum_{j=k+1}^{K} m_{j}$. Theorem 3.3.4 can be used to generate draws from this $G D$ distribution. 
6. Sample a new value for the precision parameter $\gamma$. If a Gamma prior is chosen, i.e. $\pi(\gamma) \stackrel{D}{=} G a(a, b)$, then the posterior conditional distribution for $\gamma$ simplifies to

$$
\begin{aligned}
\pi(\gamma \mid \mathbf{p}) & \propto \pi(\mathbf{p} \mid \gamma) \pi(\gamma) \\
& \propto f(\mathbf{p} \mid \mathbf{1}, \gamma \cdot \mathbf{1}) \cdot \pi(\gamma) \\
& \propto \gamma^{K-1} p_{K}^{\gamma} \cdot \gamma^{a-1} e^{-b \gamma} \\
& \propto \gamma^{a+K-2} e^{-\left(b-\log p_{K}\right) \gamma}
\end{aligned}
$$

This implies that $(\gamma \mid \mathbf{p}) \sim G a\left(a+K-1, b-\log p_{K}\right)$.

Ishwaran and Zarepour (2000) offer a detailed discussion of the unique benefits and disadvantages of the two blocked sampling schemes discussed above. Several of their comments are pertinent to the discussion of sampler choice. They note, for example, that the mixture model based on the Truncated Approximation $\mathcal{G}_{K}$ is a more accurate approximation of the DPMM than the mixture model based on the Weak Limit Approximation $\mathcal{G}_{K_{W}}$. Additionally, the TA mixture model has a more easily computable $\mathcal{L}_{1}$ bound on the marginal distribution error (3.3.1.1) compared to the WLA mixture model (3.3.11). A potential disadvantage of the TA mixture model and the resultant blocked sampler is the propensity for numerical problems when sampling from the conditional posterior for $\gamma$. Ishwaran and Zarepour do discuss possible options for cases where this is a problem.

A final note is worth making before proceeding. It was stated previously that either of the samplers based on the stick-breaking interpretation of the DP discussed here would be preferable for use throughout this work because of the non-conjugacy problem inherent in the Polya urn sampler. While it is true that the Polya urn sampler depends on the evaluation of an integral that is not in general analytically tractable outside of the case of conjugacy, it should be emphasized that the stick-breaking sampler still requires a sample from $\pi\left(\theta_{k}^{*} \mid \mathbf{C}, \psi, \mathbf{X}\right) \propto d H\left(\theta_{k}^{*}\right) \prod_{\left\{i: C_{i}=k\right\}} f\left(X_{i} \mid \theta_{k}^{*}, \psi\right)$. In the case of non-conjugacy, Step 1 of the stick-breaking sampler will require the use of a MH step to take advantage of the potential to block sample $\boldsymbol{\theta}^{*}$. 


\subsection{Posterior Inference for $\mathcal{G}$ and its Functionals}

An additional benefit of the blocked Gibbs sampler just presented is the ease with which the resulting posterior samples facilitate analysis of the mixture distribution $\mathcal{G}$ and its functionals. Recall that iteratively drawing from the conditional distributions in steps (1)-(6) above produces values drawn from the posterior distribution $\pi\left(\boldsymbol{\theta}^{*}, \mathbf{C}, \psi, \boldsymbol{\eta}, \mathbf{p}, \gamma \mid \mathbf{X}\right)$. Assuming we draw $Q$ posterior MCMC samples, we label these posterior draws $\left\{\tilde{\boldsymbol{\theta}}_{q}^{*}, \tilde{\mathbf{C}}_{q}, \tilde{\psi}_{q}, \tilde{\boldsymbol{\eta}}_{q}, \tilde{\mathbf{p}}_{q}, \tilde{\gamma}_{q}\right\}$ for $q=1, \ldots, Q$. Note that each posterior draw also defines a random probability measure

$$
\tilde{G}_{q}(\cdot)=\sum_{k=1}^{K} \tilde{p}_{q, k} \delta_{\tilde{\theta}_{q, k}^{*}}(\cdot)
$$

such that the set $\left\{\tilde{G}_{1}, \ldots, \tilde{G}_{M}\right\}$ forms a sample from $\mathcal{G}_{K}(\cdot \mid \mathbf{X})$. As a consequence, the set $\left\{\hat{G}_{1}, \ldots, \hat{G}_{Q}\right\}$ can be used for inference on both $\mathcal{G}(\cdot \mid \mathbf{X})$ and its functionals directly (Ishwaran \& James, 2001).

In future chapters we will be particularly interested in applying the DPMM methodology to the Threshold Regression model. As such, we will be interested in the posterior distributions of functionals of the event time distribution. Let $f\left(x \mid \mathcal{G}_{K}\right)$ be the density function for the data under model (3.3.12) conditional on the specified stick-breaking measure, i.e.

$$
f\left(x \mid \mathcal{G}_{K}\right)=\int f(x \mid \theta, \psi) \pi(\psi) d \mathcal{G}_{K}(\theta)
$$

and let $F^{*}\left(f\left(x \mid \mathcal{G}_{K}\right)\right)$ be a linear functional of that density, e.g. the survival or hazard function. Then the set $\left\{F^{*}\left(f\left(x \mid \tilde{G}_{q}\right)\right)\right\}_{q=1, \ldots, Q}$ is a sample from the posterior distribution $\left[F^{*}\left(f\left(x \mid \mathcal{G}_{K}\right)\right) \mid \mathbf{X}\right]$. This representation is particularly useful because $f\left(x \mid \hat{G}_{q}\right)$ is easy to compute:

$$
f\left(x \mid \tilde{G}_{q}\right)=\sum_{k=1}^{K} \tilde{p}_{q, k} f\left(x \mid \tilde{\theta}_{q, k}^{*}\right) .
$$

Utilizing this method we will be able to make draws from the posterior distribution for the survival, hazard, and cumulative hazard functions, etc. 


\subsection{Use of the Dirichlet Process in Survival Analysis}

Before closing this chapter we take time to briefly review the literature on past uses of the Dirichlet process in survival analysis. The earliest such work applied the DP directly to the event time distribution resulting in a Bayesian analog of the Kaplan-Meier method. Susarla and Ryzin (1976) appear to be the first to derive a Bayes estimator of $S(t)=1-F(t)$ under the Dirichlet Process prior and in the presence of independent right censoring. Kuo and Smith (1992) discuss estimation of the survival function under the DP prior in the presence of interval censoring. Ibrahim, Chen, and Sinha (2001) offer a thorough summary of these and similar methods.

The DP has also been widely employed in Accelerated Failure Time (AFT) models. Much of this research has focused on employing the DP as a nonparametric model for the baseline survival distribution. This is similar in theme to the Cox PH model which makes a "nonparametric" assumption on the hazard function. Christensen and Johnson (1988) and Hanson and Johnson (2004) are two prominent examples of this work. In a similar vein, Hanson (2006) considers AFT models where the baseline survival distribution is modeled as a Dirichlet process mixture of gamma densities. Walker and Mallick (1997), on the other hand, consider a Bayesian AFT model where the distribution of the subject specific frailties is modeled using the DP prior. This latter methodology is more analogous to the DPMM in that the nonparametric distribution mixes over latent "groups" under a parametric model. Unlike the DPMM, though, the model from Walker and Mallick requires that the nonparametric distribution over the frailties be centered on 0 due to identifiability issues. This requirement for centering necessitates the use of a generalization of the Dirichlet Process called a Polya Tree which more easily accommodates this restriction.

Kottas (2006) notes that, despite the broader popularity of DPMMs, surprisingly little attention has been paid to DPMMs supported on $\mathbb{R}^{+}$with applications in survival analysis. Kottas speculates that this lack of attention is a consequence of the difficulty in estimating functionals of the survival time distribution when direct draws from the posterior of the mixture distribution $\mathcal{G}$ are not available, as in the Polya urn sampler. As mentioned earlier, 
functionals such as the hazard and cumulative hazard functions have played an important role in survival analysis and appropriate methods for estimation of these quantities are vital if DPMMs are to become prominent in this field. Kottas (2006) did develop a method to estimate functionals of Dirichlet Process mixtures which can be applied under the Polya urn sampler and demonstrates this method in the context of DP mixtures of the Weibull distribution. Ishwaran and James (2001) discuss similar methods. Despite this advance, the DP mixture methods developed in future chapters will limit considerations to the stickbreaking sampler where it is easier to sample the posteriors for functionals common in survival analysis. 


\section{Chapter 4 \\ The Dirichlet Process Mixture FOR THRESHOLD REgRESSION}

Often times success doesn't come from strength, but from flexibility and adaptability.

- Debasish Mridha MD

The preceding two chapters have respectively been concerned with foundational results for Threshold Regression and the Dirichlet Process Mixture model. The current chapter proposes a flexible survival analysis methodology which combines these two techniques and models survival data as coming from DP-mixtures of the First Hitting Time distribution.

As a motivating example for the work in this chapter, consider long-term survival for cancer patients who are randomized to undergo different surgical procedures to remove malignant tissue. As a first approximation, it seems reasonable to assume that the latent process assumptions underlying the IG distribution hold within individual subjects post surgery; i.e., patients have subject-specific initial health and health trend after surgery. Given this, we should like to identify the immediate and long term effects on health of the various procedures under consideration and we could use the standard TR model (2.4.1) to make such comparisons. In this hypothetical setting, however, there is likely to exist heterogeneity in subject-specific responses to treatment. In other words, there is likely a spectrum of responses to treatment and such diversity would be expected to result in data which violate the IG assumption. In Chapter 2 we introduced two possible methods to account for such latent heterogeneity; the random effects model (2.5.13) and the finite mix- 
ture model (2.5.16). While both models have received attention in the literature, neither are without fairly profound weaknesses. Consider, for example, the possibility that some patients may have tumors which have recently metastasized so that this metastasis is unknown. Such latent heterogeneity would likely induce a multi-modal response to treatment because subjects with unknown metastatic tumors are likely to have profoundly different responses compared to those without. Such multi-modal heterogeneity is unlikely to be well modeled via the random effects model (2.5.13). On the other hand, we may be uncomfortable with a strict assumption about the number of latent clusters which might be induced by unmeasured metastasis; an assumption which is necessary in standard finite mixture methods (2.5.16). In response, we consider assigning the flexible Dirichlet Process prior to the Threshold Regression parameters in order to greatly reduce assumptions about any latent heterogeneity.

We begin the current chapter by introducing the proposed model and clarifying notation. A detailed discussion of model fitting via MCMC follows. Strengths of the proposed methodology are highlighted by results from a pertinent simulation study. Finally, the methodology is applied to data from an in vivo carcinogenicity study conducted by the National Toxicology Program.

\subsection{The DPM-TR Model}

Our immediate goal is to apply the DPMM methodology (3.1.2) to the TR model, which can be done in standard fashion. As a first step in developing our proposed model we consider the general Threshold Regression model which allows for subject-specific covariate effects, i.e.

$$
\begin{aligned}
f\left(t_{i} \mid x_{0 i}, \mu_{i}\right) & =\frac{x_{0 i}}{\sqrt{2 \pi t_{i}^{3}}} \exp \left\{-\frac{\left(x_{0 i}+\mu_{i} t_{i}\right)^{2}}{2 t_{i}}\right\} I\left\{t_{i}>0\right\} \\
g\left(x_{0 i}\right) & =\mathbf{v}_{i}^{\top} \boldsymbol{\alpha}_{i} \\
h\left(\mu_{i}\right) & =\mathbf{w}_{i}^{\top} \boldsymbol{\beta}_{i} .
\end{aligned}
$$


As discussed in Chapter 2, this model allows us to analyze both immediate $\left(\boldsymbol{\alpha}_{i}\right)$ and long term $\left(\boldsymbol{\beta}_{i}\right)$ effects of covariates. We wish to make minimal assumptions about differences between subjects in these covariate effects and so assume that subject-specific regression coefficients are samples from the Dirichlet Process. Additionally, it is often the case in mixture models that latent heterogeneity is thought to exist in only a subset of parameters. We can accommodate such modeling choices by specifying a subset of regression coefficients $\theta_{i}=\left\{\boldsymbol{\alpha}_{i}, \boldsymbol{\beta}_{i}\right\}$, corresponding to covariates $\left\{\mathbf{v}_{h i}, \mathbf{w}_{h i}\right\}$, which are allowed to exhibit heterogeneity under a specified DP mixture prior. The remaining covariates, $\left\{\mathbf{v}_{f i}, \mathbf{w}_{f i}\right\}$ are associated with fixed parameters $\psi=\{\mathbf{a}, \mathbf{b}\}$ and we call the specification of $\left\{\mathbf{v}_{h i}, \mathbf{v}_{f i}, \mathbf{w}_{h i}, \mathbf{w}_{f i}\right\}$ the heterogeneity structure. Given a heterogeneity structure, a general specification of our proposed model is accomplished by assigning appropriate priors to $\psi=\{\mathbf{a}, \mathbf{b}\}$ and to the DP mixture distribution controlling $\theta_{i}=\left\{\boldsymbol{\alpha}_{i}, \boldsymbol{\beta}_{i}\right\}$. As an example, we will utilize the following model specification for a later simulation study and data example:

$$
\begin{aligned}
& T_{i} \mid \mathbf{v}_{i}, \mathbf{w}_{i} \stackrel{i n d}{\sim} I G\left(\exp \left\{\left[\begin{array}{c}
\mathbf{v}_{h i} \\
\mathbf{v}_{f i}
\end{array}\right]^{\top}\left[\begin{array}{l}
\boldsymbol{\alpha}_{i} \\
\mathbf{a}
\end{array}\right]\right\},\left[\begin{array}{l}
\mathbf{w}_{h i} \\
\mathbf{w}_{f i}
\end{array}\right]^{\top}\left[\begin{array}{l}
\boldsymbol{\beta}_{i} \\
\mathbf{b}
\end{array}\right]\right), \\
& \theta_{i}=\left[\begin{array}{c}
\boldsymbol{\alpha}_{i} \\
\boldsymbol{\beta}_{i}
\end{array}\right] \stackrel{i n d}{\sim} G \\
& \psi=\left[\begin{array}{l}
\mathbf{a} \\
\mathbf{b}
\end{array}\right] \sim N\left(\boldsymbol{\mu}_{\psi}, \boldsymbol{\Sigma}_{\psi}\right) \\
& G \sim D P(\gamma H), \\
& \gamma \sim \mathrm{Ga}\left(a_{\gamma}, b_{\gamma}\right), \\
& H \stackrel{D}{=} N(\boldsymbol{\mu}, \boldsymbol{\Sigma}), \\
& (\boldsymbol{\mu}, \boldsymbol{\Sigma}) \sim \pi(\boldsymbol{\mu}, \boldsymbol{\Sigma}) .
\end{aligned}
$$

We refer to Model 4.1.2 as the Dirichlet Process Mixture for Threshold Regression (DPMTR) model.

The above model statement is, of course, not fully specified. Typically one would want to specify priors on $\psi$ and $(\boldsymbol{\mu}, \boldsymbol{\Sigma})$ which are fairly vague. We suggest that priors be based on 
estimates $\hat{x}_{0}$ and $\hat{\mu}$ from fitting the IG distribution, i.e. $I G\left(x_{0}, \mu\right)$, to the data in question. Then, because of the relationship between $\left(x_{0 i}, \mu_{i}\right)$ and the first two moments of $T_{i}$, i.e.

$$
\begin{aligned}
x_{0 i} & =\operatorname{var}\left(T_{i}\right) / E\left[T_{i}\right], \\
\mu_{i} & =-\operatorname{var}\left(T_{i}\right) / E^{2}\left[T_{i}\right],
\end{aligned}
$$

we would expect that $\hat{x}_{0}$ and $\hat{\mu}$ will usually convey insight at least about the magnitude of the sample average parameters $\left(\bar{x}_{0}, \bar{\mu}\right)=\frac{1}{n} \sum\left(x_{0 i}, \mu_{i}\right)$. Assuming covariates are scaled appropriately, priors for $\psi$ and $\boldsymbol{\mu}$ centered on $\mathbf{0}$ and with large variance compared to $\max \left\{\log \left(\hat{x}_{0}\right), \hat{\mu}\right\}$ will usually contribute little to the posterior. We will generally have little prior information regarding the variability of subject-specific parameters so a diffuse prior on $\boldsymbol{\Sigma}$ should usually be chosen. As an example of the above scheme we briefly consider the National Toxicology Program data example which will be introduced in more detail later. Fitting the $\operatorname{IG}\left(x_{0}, \mu\right)$ distribution to these data gives $\left(\hat{x}_{0}, \hat{\mu}\right)=(3.889,-0.049)$, so the following priors would be expected to be quite diffuse:

$$
\begin{aligned}
& \psi \sim N(\mathbf{0}, 10000 \cdot \mathbf{I}), \\
& \boldsymbol{\mu} \sim N(\mathbf{0}, 10000 \cdot \mathbf{I}), \\
& \boldsymbol{\Sigma}=\operatorname{diag}\left\{\sigma_{1}^{2}, \ldots, \sigma_{d}^{2}\right\} \\
& \sigma_{j}^{2} \stackrel{i n d}{\sim} \operatorname{Inv}-\operatorname{Gamma}\left(10^{-5}, 10^{-5}\right) .
\end{aligned}
$$

Before continuing, we should note one peculiarity about the priors in (4.1.4). Specifically, note that the variance $\boldsymbol{\Sigma}$ in the base prior $H$ is assumed diagonal. In light of our comment that we generally wish to specify vague priors on $\boldsymbol{\Sigma}$, diagonality here might seem like an unnecessarily strong assumption. This restriction, however, is justifiable for two reasons. First, even though $\boldsymbol{\Sigma}$ is assumed diagonal, the DP prior still allows the mixture distribution $G$ to have a non-diagonal covariance matrix. In essence, the diagonality assumption is expressing an a priori belief that the subject-specific coefficients are not strongly dependent. This being the case, diagonality in $\boldsymbol{\Sigma}$ is not as strong an assumption as it may seem. Secondly, the diagonality assumption acts as a regularization prior, facilitating numerical 
stability in the MCMC sampling scheme used to fit the DPM-TR model. In contrast, the Inverse-Wishart (IW) distribution was also considered as a possible prior for $\boldsymbol{\Sigma}$. MCMC chains for the DPM-TR model utilizing the IW prior often mixed more slowly than those for equivalent models with $\pi(\boldsymbol{\Sigma})$ as in (4.1.4). Together these two observations suggest that the stated prior on $\boldsymbol{\Sigma}$ results in a significant computational benefit for little cost in model flexibility.

\subsection{Model Selection Criteria}

While it is fairly straightforward to specify a complete DPM-TR model, the question of model selection for the proposed methodology is somewhat more difficult. Model selection in the context of Dirichlet Process Mixtures takes on additional complexity due to the reality that a heterogeneity structure must be selected in addition to covariate selection. In other words, it would be desirable to have model selection tools that can accurately discriminate between potential sources of latent heterogeneity. In the simulation which follows, we explore the possibility that the well-known Pseudo Marginal Likelihood (PML) criterion can facilitate this type of model discrimination. While a full discussion of PML methods will not be possible here, the reader is directed to Gelfand and Dey (1994) who develop several important theoretical results. For our current purposes it will be sufficient to state that the PML is a "likelihood" of the observed data conditional on "leave one out" cross-validation. Stated symbolically, the PML for data $\mathbf{y}$ under model $M_{l}$ can be expressed as

$$
\operatorname{PML}\left(\mathbf{y}, M_{l}\right)=\prod_{i=1}^{n} \hat{f}\left(y_{i} \mid \mathbf{y}_{(i)}, M_{l}\right)
$$

where $\hat{f}\left(y_{i} \mid \mathbf{y}_{(i)}, M_{l}\right)$ is the density function for datum $y_{i}$ conditional on model $M_{l}$ fit to all the data except $y_{i}$, i.e. $\mathbf{y}_{(i)}=\left\{y_{j}: j \neq i\right\}$. The PML is particularly useful for our purposes because it can be calculated from a single MCMC chain fitting Model $M_{l}$ to the full dataset. As such we need not incur the computational cost of fitting the model $M_{l}$ to all $n$ datasets 
$\mathbf{y}_{(i)}, i=1, \ldots, n$. Gelfand and Dey (1994) show that the PML can be rewritten as

$$
\operatorname{PML}\left(\mathbf{y}, M_{l}\right)=\prod_{i=1}^{n} Q_{l}\left\{\sum_{q}^{Q_{l}} \frac{1}{f\left(y_{i} \mid \tilde{\boldsymbol{\theta}}_{l q}, M_{l}\right)}\right\}^{-1}
$$

where $\tilde{\boldsymbol{\theta}}_{l q}$ is the $q^{\text {th }}$ of $Q_{l}$ MCMC samples of the posterior distribution of $\boldsymbol{\theta}$ under model $M_{l}$.

The PML can then be used in a variety of ways for model selection. The simplest use of PML is to rank all considered models by their PML and consider the model with the highest PML to be best. As with other information criteria type methods such as AIC and BIC, this approach does not tell you if the best model is actually significantly better than competing models. If we wish to answer this latter question, we can use the PML to compare multiple models using the "pseudo Bayes Factor"

$$
\operatorname{PBF}\left(M_{1}, M_{2} \mid \mathbf{y}\right)=\frac{\operatorname{PML}\left(\mathbf{y}, M_{1}\right)}{\operatorname{PML}\left(\mathbf{y}, M_{2}\right)}
$$

which we can interpret as the ratio of posterior probabilities for the two models. There is a wide range of suggestions for appropriate uses of Bayes Factors in analysis. For example, in our simulation study it will suffice to follow the suggestion of Kass and Raftery (1995) by concluding that there is strong evidence for model $M_{1}$ over model $M_{2}$ if $\operatorname{PBF}\left(M_{1}, M_{2} \mid \mathbf{y}\right)>$ 20.

\subsection{The Blocked Sampler}

Because the Threshold Regression model does not in general have conjugate priors for regression coefficients $\boldsymbol{\alpha}$ and $\boldsymbol{\beta}$, it will be necessary to employ one of the blocked samplers discussed in Section 3.3. As in the preceding chapter, it will be beneficial to restate the 
approximate DPM-TR model in terms of cluster membership $\mathbf{C}=\left(C_{1}, \ldots, C_{n}\right)$, i.e.

$$
\begin{aligned}
& T_{i} \mid \mathbf{v}_{i}, \mathbf{w}_{i} \stackrel{i n d}{\sim} I G\left(\exp \left\{\left[\begin{array}{l}
\mathbf{v}_{h i} \\
\mathbf{v}_{f i}
\end{array}\right]^{\top}\left[\begin{array}{l}
\boldsymbol{\alpha}_{i} \\
\mathbf{a}
\end{array}\right]\right\},\left[\begin{array}{l}
\mathbf{w}_{h i} \\
\mathbf{w}_{f i}
\end{array}\right]^{\top}\left[\begin{array}{l}
\boldsymbol{\beta}_{i} \\
\mathbf{b}
\end{array}\right]\right), \\
& \theta_{i}=\left\{\boldsymbol{\alpha}_{i}, \boldsymbol{\beta}_{i}\right\}, \\
& \psi=\{\mathbf{a}, \mathbf{b}\} \sim N\left(\boldsymbol{\mu}_{\psi}, \boldsymbol{\Sigma}_{\psi}\right), \\
& \theta_{i} \mid C_{i}=\theta_{C_{i}}^{*}, \\
& C_{i} \mid \mathbf{p} \stackrel{i n d}{\sim} \operatorname{Multinomial}(\mathbf{p}), \\
& \left(\mathbf{p}, \boldsymbol{\theta}^{*}\right) \sim \pi(\mathbf{p} \mid \gamma) \times d H^{K}\left(\boldsymbol{\theta}^{*}\right), \\
& H \stackrel{D}{=} N(\boldsymbol{\mu}, \boldsymbol{\Sigma}), \\
& \boldsymbol{\mu} \sim N\left(\boldsymbol{\mu}_{0}, \boldsymbol{\Sigma}_{0}\right) \\
& \boldsymbol{\Sigma}=\operatorname{diag}\left\{\sigma_{1}^{2}, \ldots, \sigma_{d}^{2}\right\}, \\
& \sigma_{j}^{2} \stackrel{i i d}{\sim} I G\left(a_{0}, b_{0}\right) \\
& \gamma \sim \mathrm{Ga}\left(a_{\gamma}, b_{\gamma}\right)
\end{aligned}
$$

where

1. $C_{i}=k \Longleftrightarrow \theta_{i}=\theta_{k}^{*}$, i.e. $C_{i}$ represents cluster assignment for subject $i$.

2. $d H^{K}\left(\boldsymbol{\theta}^{*}\right)=\prod_{k}^{K} d H\left(\theta_{k}^{*}\right)$ is the joint distribution of the cluster specific parameters under the base prior $H$.

3. $\pi(\mathbf{p} \mid \gamma)$ represents the prior of $\mathbf{p}$ under the assumptions of (3.3.5).

4. $\boldsymbol{\eta}=\{\boldsymbol{\mu}, \boldsymbol{\Sigma}\}$.

With the model restatement in (3.3.12), we can derive expressions for the conditional posterior distributions of most model parameters fairly easily.

We begin by drawing a single MCMC sample from the conditional posterior $\pi\left(\boldsymbol{\theta}^{*}, \mathbf{C}, \psi, \boldsymbol{\eta} \mid \mathbf{p}, \gamma, \mathbf{X}\right)$ using the following steps:

1. Sample all cluster-specific regression vectors $\left\{\theta_{1}^{*}, \ldots, \theta_{K^{*}}^{*}\right\}$ conditional on cluster membership $\mathbf{C}=\left\{C_{1}, \ldots, C_{n}\right\}$. Recalling that subjects are assumed independent conditional on their subject specific parameter values, the following expression easily 
follows

$$
\pi\left(\theta_{k}^{*} \mid \mathbf{C}, \psi, \mathbf{t}, \mathbf{d}\right) \propto d H\left(\theta_{k}^{*}\right) \prod_{\left\{i: C_{i}=k\right\}} L\left(\boldsymbol{\alpha}_{k}, \boldsymbol{\beta}_{k} \mid t_{i}, d_{i}, \mathbf{a}, \mathbf{b}\right)
$$

where $\left(\boldsymbol{\alpha}_{k}, \boldsymbol{\beta}_{k}\right)$ represent all cluster specific regression parameters for group $k$. Computation of the likelihood $L$ is equivalent to that for the TR model (2.3.9) except limited to data currently assigned to cluster $k$, i.e.

$$
\begin{aligned}
& L\left(\boldsymbol{\alpha}_{k}, \boldsymbol{\beta}_{k} \mid t_{i}, d_{i}, \mathbf{a}, \mathbf{b}\right)=\left[f\left(t_{i} \mid \exp \left\{\left[\begin{array}{c}
\mathbf{v}_{h i} \\
\mathbf{v}_{f i}
\end{array}\right]^{\top}\left[\begin{array}{l}
\boldsymbol{\alpha}_{k} \\
\mathbf{a}
\end{array}\right]\right\},\left[\begin{array}{l}
\mathbf{w}_{h i} \\
\mathbf{w}_{f i}
\end{array}\right]^{\top}\left[\begin{array}{c}
\boldsymbol{\beta}_{k} \\
\mathbf{b}
\end{array}\right]\right)\right]^{d_{i}} \\
& \times\left[S\left(t_{i} \mid \exp \left\{\left[\begin{array}{c}
\mathbf{v}_{h i} \\
\mathbf{v}_{f i}
\end{array}\right]^{\top}\left[\begin{array}{c}
\boldsymbol{\alpha}_{k} \\
\mathbf{a}
\end{array}\right]\right\},\left[\begin{array}{l}
\mathbf{w}_{h i} \\
\mathbf{w}_{f i}
\end{array}\right]^{\top}\left[\begin{array}{c}
\boldsymbol{\beta}_{k} \\
\mathbf{b}
\end{array}\right]\right)\right]^{1-d_{i}} \text {. }
\end{aligned}
$$

Note that in the case that cluster $j$ is empty (i.e. $\left\{i: C_{i}=k\right\}=\varnothing$ ) the likelihood contribution of the data is 1 and $\left(\theta_{k}^{*} \mid \mathbf{C}, \psi, \mathbf{X}\right) \sim H$. Iteratively sample from (3.3.13) for $k=1, \ldots, K$.

It will here be necessary to make a few fairly involved comments regarding sampling of $\theta_{k}^{*}$. Due to a lack of conjugacy for the TR model, both in general and especially in the presence of censoring, we use a Random Walk Metropolis (RWM) algorithm to draw samples for $\theta_{k}^{*}$. Given the form of (4.3.2), one has two choices for methods to perform this sample: (1) block sample the whole vector $\theta_{k}^{*}$, or (2) sample each element of $\theta_{k}^{*}$ one at a time. While the second scheme is easier to code, we opt for the first option because TR parameters can be shown to be correlated, rendering block sampling potentially more efficient.

A second observation worth noting is that appropriate proposal variances for $\theta_{k}^{*}$, conditional on cluster membership, will differ between clusters due to their varying size. Consider, for example, that a proposal variance $\Sigma_{\text {prop }}$ which allows for efficient exploration of the posterior of $\theta_{k}^{*}$ where $k$ is a cluster with 10 subjects will likely be quite inefficient at exploring the equivalent posterior of $\theta_{j}^{*}$ where $j$ is a cluster with 1,000 subjects. This difference in efficiency is largely due to the fact that the variance 
of $\theta_{k}^{*}$ is roughly 2 orders of magnitude larger than the variance of $\theta_{j}^{*}$, i.e.

$$
\operatorname{var}\left(\theta_{k}^{*}\right) \approx 10^{2} \cdot \operatorname{var}\left(\theta_{j}^{*}\right)
$$

For this reason we will implement the $\mathrm{MH}$ step for each $\theta_{k}^{*}$ with a proposal variance which is inversely proportional to the current size of the cluster, i.e.

$$
\Sigma_{\text {prop }, k}=\frac{\Sigma_{\text {prop }}}{\sum I\left\{C_{i}=k\right\}}
$$

where $\Sigma_{\text {prop }}$ acts as a "base" proposal variance. That this proposed sampling scheme converges to the correct stationary distribution follows from the fact that the sampling step is actually a simple $\mathrm{MH}$ sample conditional on $\mathbf{C}$. As such the standard results for the MH algorithm apply.

Finally, a few comments regarding methods to establish the "base" proposal variance $\Sigma_{\text {prop }}$ are in order. During burn-in, $\Sigma_{\text {prop }}$ is adjusted to be proportional to the empirical variance of all preceding burn-in samples of the value $\bar{\theta}=E\left[\theta_{i}\right]=\sum_{k=1}^{K^{*}} p_{k} \theta_{k}^{*}$. This allows the diagonal elements of $\Sigma_{\text {prop }}$ to have appropriate magnitudes relative to one another. We also recommend adjusting $\Sigma_{\text {prop }}$ by a multiplicative factor every 100 burn-in steps based on the empirical acceptance rate of the preceding 100 samples so that the $\mathrm{MH}$ acceptance rate is in the interval [0.3, 0.35]. Work from Roberts and Rosenthal (2001) suggests that MH sampling with acceptance rates in this range should be appropriate for a variety of target distributions across a range of dimensions. In our experience the stated range for acceptance rates has worked well in all examples we have explored. $\Sigma_{\text {prop }}$ is not adapted after burn-in so as not to necessitate justification of the ergodicity of the resulting chain beyond those standard for the $\mathrm{MH}$ sampler.

2. Sample new values for the shared parameters $\psi=\{\mathbf{a}, \mathbf{b}\}$. Straightforward application of Bayes' Theorem gives a form similar to (4.3.2):

$$
\left(\psi \mid \boldsymbol{\theta}^{*}, \mathbf{C}, \mathbf{t}, \mathbf{d}\right) \propto \pi(\psi) \prod_{i=1}^{n} L\left(\mathbf{a}, \mathbf{b} \mid t_{i}, d_{i}, \boldsymbol{\alpha}_{C_{i}}, \boldsymbol{\beta}_{C_{i}}\right) .
$$

Here, $L\left(\mathbf{a}, \mathbf{b} \mid t_{i}, d_{i}, \boldsymbol{\alpha}_{C_{i}}, \boldsymbol{\beta}_{C_{i}}\right)$ is equal to $L\left(\boldsymbol{\alpha}_{C_{i}}, \boldsymbol{\beta}_{C_{i}} \mid t_{i}, d_{i}, \mathbf{a}, \mathbf{b}\right)$ defined in (4.3.3). RWM 
samples can be taken from (4.3.6) in a manner completely analogous to Step 1.

3. Sample new values for cluster membership conditional on cluster-specific parameters $\boldsymbol{\theta}^{*}$, shared parameters $\psi$, and stick-breaking weights $\mathbf{p}$. Sample from

$$
\pi\left(C_{i} \mid \boldsymbol{\theta}^{*}, \mathbf{p}, \psi, \mathbf{t}, \mathbf{d}\right) \stackrel{D}{=} \operatorname{Multinomial}\left(\mathbf{p}_{i}^{*}\right)
$$

for each subject $i=1, \ldots, n$, where

$$
\begin{aligned}
& \mathbf{p}_{i}^{*}=\left\{p_{i k}^{*}\right\}_{k=1}^{K^{*}}, \\
& p_{i k}^{*} \propto p_{k} \cdot L\left(\boldsymbol{\alpha}_{k}, \boldsymbol{\beta}_{k}, \mathbf{a}, \mathbf{b} \mid t_{i}, d_{i}\right) .
\end{aligned}
$$

The justification for the form of (3.3.15) can be seen by considering the truncated stick-breaking representation of the Multinomial distribution.

$$
\begin{aligned}
\pi\left(C_{i} \mid \mathbf{p}, t_{i}, d_{i}\right) & \propto \pi\left(C_{i} \mid \mathbf{p}\right) \cdot L\left(\theta_{C_{i}}^{*} \mid \psi, t_{i}, d_{i}\right) \\
& \propto \sum_{k=1}^{K^{*}} p_{k} \delta_{\theta_{k}^{*}}(\cdot) \cdot L\left(\boldsymbol{\alpha}_{k}, \boldsymbol{\beta}_{k}, \mathbf{a}, \mathbf{b} \mid t_{i}, d_{i}\right) \\
& \propto \sum_{k=1}^{K^{*}}\left(p_{k} \cdot L\left(\boldsymbol{\alpha}_{k}, \boldsymbol{\beta}_{k}, \mathbf{a}, \mathbf{b} \mid t_{i}, d_{i}\right)\right) \delta_{\theta_{k}^{*}}(\cdot) .
\end{aligned}
$$

Again, $L\left(\boldsymbol{\alpha}_{k}, \boldsymbol{\beta}_{k}, \mathbf{a}, \mathbf{b} \mid t_{i}, d_{i}\right)$ is the same as in (4.3.3).

4. Sample new values for the hyper-parameters $\boldsymbol{\eta}$ controlling the baseline prior $H$. This step is a direct application of Bayes' theorem because we have a sample $\boldsymbol{\theta}^{*}$ from $H$ :

$$
\pi\left(\boldsymbol{\eta} \mid \boldsymbol{\theta}^{*}, \psi, \mathbf{C}, \mathbf{t}, \mathbf{d}\right) \equiv \pi\left(\boldsymbol{\eta} \mid \boldsymbol{\theta}^{*}, K\right) \propto \pi(\boldsymbol{\eta}) \prod_{k=1}^{K^{*}} d H\left(\theta_{k}^{*} \mid \boldsymbol{\eta}\right) .
$$

In our sampler we assume that $H$ is the normal distribution with parameters $\boldsymbol{\mu}$ and $\boldsymbol{\Sigma}=\operatorname{diag}\left\{\sigma_{1}^{2}, \ldots, \sigma_{d}^{2}\right\}$, which are assumed a prior independent. The conjugate normal prior for $\boldsymbol{\mu}$ is used so that the conditional posterior for $\boldsymbol{\mu}$ is given as:

$$
\begin{gathered}
\left(\boldsymbol{\mu} \mid \boldsymbol{\Sigma}, \boldsymbol{\theta}^{*}, \boldsymbol{\mu}_{0}, \boldsymbol{\Sigma}_{0}\right) \sim N\left(\boldsymbol{\mu}_{p}, \boldsymbol{\Sigma}_{p}\right) \\
\boldsymbol{\mu}_{p}=\left(\boldsymbol{\Sigma}_{0}^{-1}+n \boldsymbol{\Sigma}^{-1}\right)^{-1}\left(\boldsymbol{\Sigma}_{0}^{-1} \boldsymbol{\mu}_{0}+n \boldsymbol{\Sigma}^{-1} \overline{\boldsymbol{\theta}}^{*}\right) \\
\boldsymbol{\Sigma}_{p}=\left(\boldsymbol{\Sigma}_{0}^{-1}+n \boldsymbol{\Sigma}^{-1}\right)^{-1}
\end{gathered}
$$


Our prior for $\sigma_{j}^{2}$ is also conjugate and we again employ the standard Gibbs update:

$$
\begin{aligned}
& \left(\sigma_{j}^{2} \mid \boldsymbol{\mu}, \boldsymbol{\theta}^{*}, a_{0}, b_{0}\right) \sim \operatorname{Inv-Gamma}\left(a_{p}, b_{p}\right), \\
& a_{p}=a_{0}+K / 2, \\
& b_{p}=b_{0}+\frac{\sum\left(\theta_{i j}^{*}-\mu_{j}\right)^{2}}{2} \text {. }
\end{aligned}
$$

Steps (1)-(4) are sufficient to sample from the conditional posterior $\pi\left(\boldsymbol{\theta}^{*}, \mathbf{C}, \psi, \boldsymbol{\eta} \mid \mathbf{p}, \gamma, \mathbf{t}, \mathbf{d}\right)$. A single Gibbs step sampling from the full posterior $\pi\left(\boldsymbol{\theta}^{*}, \mathbf{C}, \psi, \boldsymbol{\eta}, \mathbf{p}, \gamma \mid \mathbf{t}, \mathbf{d}\right)$ can be completed by subsequently sampling from $\pi\left(\mathbf{p}, \gamma \mid \boldsymbol{\theta}^{*}, \mathbf{C}, \psi, \boldsymbol{\eta}, \mathbf{t}, \mathbf{d}\right)$.

5. Sample new values for the random weights $\mathbf{p}$ according to the following algorithm:

$$
\begin{aligned}
p_{k} & =V_{k} \prod_{j=1}^{k-1}\left(1-V_{j}\right) \text { for } k=1, \ldots, K^{*}-1, \\
p_{K^{*}} & =1-\sum_{k=1}^{K^{*}-1} p_{k}, \\
V_{k} & \stackrel{i n d}{\sim} \operatorname{Beta}\left(a_{k}, b_{k}\right), \\
a_{k} & =1+m_{k} \\
b_{k} & =\gamma+M_{k},
\end{aligned}
$$

where $m_{k}=\left|\left\{i: C_{i}=k\right\}\right|$ records the number of subjects assigned to group $k$ and $M_{k}=\sum_{j=k+1}^{K} m_{j}$. This sampling scheme comes from the assumption of the TA measure (see Section 3.3.4).

6. Sample a new value for the precision parameter $\gamma$. If a Gamma prior is chosen, i.e. $\pi(\gamma) \stackrel{D}{=} \mathrm{Ga}\left(a_{\gamma}, b_{\gamma}\right)$, then the posterior conditional distribution for $\gamma$ simplifies to $(\gamma \mid \mathbf{p}) \sim G a\left(a_{\gamma}+K^{*}-1, b_{\gamma}-\log p_{K^{*}}\right)$. Because of the potential for numerical issues when calculating $\log p_{K^{*}}$, we sample from the equivalent distribution

$$
(\gamma \mid \mathbf{p}) \sim G a\left(a_{\gamma}+K^{*}-1, b_{\gamma}-\sum_{k=1}^{K^{*}-1} \log \left(1-V_{k}\right)\right),
$$

which has the simple additional requirement that the $V_{k}$ values be temporarily stored.

Repeating steps (1)-(6) for a large number of iterations $Q$ results in a Metropolis within 
Gibbs sample from the full posterior distribution for the DPM-TR model.

\subsection{Simulation Study}

In order to evaluate the performance of the proposed methodology, we applied our model and two other common Threshold Regression based methods to data generated with both measured and unmeasured heterogeneity. Below we review the data generation methods employed in this study, the fitted models, and insightful results.

\subsubsection{Simulated Data}

To begin, it will be beneficial to describe the basic framework under which all simulated data were generated. Each simulated dataset includes information for $n=600$ subjects including (1) an event time $t_{i}$ and event indicator $d_{i}$, (2) a continuous covariate $v_{i 1}$ associated with initial health $x_{0 i}$ and (3) a discrete covariate $w_{i 1}$ which is independent of $v_{i 1}$ and which is associated with health drift $\mu_{i}$.

$$
\begin{gathered}
T_{i} \mid \boldsymbol{\alpha}_{i}, \boldsymbol{\beta} \stackrel{i n d}{\sim} \operatorname{IG}\left(\mathbf{v}_{i}^{\top} \boldsymbol{\alpha}_{i}, \mathbf{w}_{i}^{\top} \boldsymbol{\beta}_{i}\right), \\
\mathbf{v}_{i}^{\top}=\left[1, v_{i}\right] \text { where } v_{i} \sim \mathrm{N}(0,1), \\
\mathbf{w}_{i}^{\top}=\left[1, w_{i}\right] \text { where } w_{i}= \begin{cases}0, & \text { if } 1 \leq i \leq 150, \\
0.2, & \text { if } 151 \leq i \leq 300, \\
0.4, & \text { if } 301 \leq i \leq 450, \\
1, & \text { if } 451 \leq i \leq 600 .\end{cases}
\end{gathered}
$$

In all, we considered 5 unique latent heterogeneity cases:

1. Case 1: Finite mixture with latent heterogeneity in both $\boldsymbol{\alpha}_{i}$ and $\boldsymbol{\beta}_{i}((\alpha, \beta)$-het)

$$
\begin{gathered}
\boldsymbol{\alpha}_{i} \sim 0.6 \cdot \delta_{(3,0.3)^{\top}}+0.4 \cdot \delta_{(2,0.1)^{\top}}, \\
\boldsymbol{\beta}_{i} \sim 0.6 \cdot \delta_{(-1,-0.5)^{\top}}+0.4 \cdot \delta_{(-3,-0.1)^{\top}} \cdot
\end{gathered}
$$


2. Case 2: Finite mixture with latent heterogeneity in $\boldsymbol{\alpha}_{i}$ only ( $\alpha$-het)

$$
\begin{gathered}
\boldsymbol{\alpha}_{i} \sim 0.6 \cdot \delta_{(3,0.3) \boldsymbol{\top}}+0.4 \cdot \delta_{(2,0.1) \boldsymbol{\top}} \\
\boldsymbol{\beta}_{i}=(-1,-0.5)^{\top}
\end{gathered}
$$

3. Case 3: Finite mixture with latent heterogeneity in $\boldsymbol{\beta}_{i}$ only ( $\beta$-het)

$$
\begin{gathered}
\boldsymbol{\alpha}_{i}=(3,0.3)^{\top} \\
\boldsymbol{\beta}_{i} \sim 0.6 \cdot \delta_{(-1,-0.5)^{\top}}+0.4 \cdot \delta_{(-3,-0.1)^{\top}}
\end{gathered}
$$

4. Case 4: No latent heterogeneity (No Het)

$$
\begin{gathered}
\boldsymbol{\alpha}_{i}=(3,0.3)^{\top}, \\
\boldsymbol{\beta}_{i}=(-1,-0.5)^{\top} .
\end{gathered}
$$

5. Case 5: Random normal intercept $\beta_{0 i}$ (Random Effect)

$$
\begin{gathered}
\boldsymbol{\alpha}_{i}=(3,0.3)^{\top}, \\
\boldsymbol{\beta}_{i} \stackrel{D}{=}\left[N\left(-1, \sigma_{\beta_{0}}^{2}\right),-0.5\right]^{\top}, \\
\sigma_{\beta_{0}}^{2}=0.3 .
\end{gathered}
$$

In addition to exploring the performance of the DPM-TR model under a variety of unique heterogeneity conditions, it is also necessary to establish that these models perform well under potentially high censoring. To simulate censoring, we assume an independent censoring process which also follows a FHT model; i.e., we let the censoring time for subject $i$ be $U_{i} \stackrel{i i d}{\sim} \operatorname{IG}\left(x_{0 u}, \mu_{u}\right)$. The observed data for subject $i$ are then the observation time $t_{i}=\min \left\{T_{i}, U_{i}\right\}$ and an event indicator $d_{i}=I\left\{T_{i} \leq U_{i}\right\}$. For all censoring processes we set $x_{0 u}$ to be the marginal expected value for initial health values in the simulation, i.e. $x_{0 u}=E\left[x_{0 i}\right]$. Two different values for $\mu_{u}$ were found for each of the 5 data cases, each associated with either $25 \%$ and $50 \%$ marginal expected censoring.

In order to have a sufficiently large study, we simulated $N_{\text {sim }}=100$ dataset from each Data Case $\times$ Censoring Level combination. In other words, we simulated $N_{\text {datasets }}=$ 
$N_{\text {cases }} \times N_{\text {censoring }} \times N_{\text {sim }}=5 \times 3 \times 100=1500$ datasets for our study.

\subsubsection{Fitted Models}

A total of six distinct survival models were fit to each simulated dataset. Three of these are DPM-TR models with different modeled heterogeneity structures. Bayesian versions of the standard TR (2.4.1) and Random Drift (2.5.1) models were also fit. Finally, the Cox proportional hazard model was also considered. While the latter cannot actually estimate the Threshold Regression parameters $(\boldsymbol{\alpha}, \boldsymbol{\beta})$, comparisons of survival function estimates between the Cox model and the FHT models, as well as the directions of estimated covariate effects, are of interest.

1. Model 1: DPM-TR with $\boldsymbol{\theta}_{i}=\left\{\boldsymbol{\alpha}_{i}, \boldsymbol{\beta}_{i}\right\}$ and $\psi=\emptyset\left(\right.$ DPM-TR $\left._{\text {full }}\right)$.

2. Model 2: DPM-TR with $\boldsymbol{\theta}_{i}=\left\{\boldsymbol{\alpha}_{i}\right\}$ and $\psi=\{\boldsymbol{\beta}\}\left(\mathrm{DPM}-\mathrm{TR}_{\alpha}\right)$.

3. Model 3: DPM-TR with $\boldsymbol{\theta}_{i}=\left\{\boldsymbol{\beta}_{i}\right\}$ and $\psi=\{\boldsymbol{\alpha}\}\left(\mathrm{DPM}-\mathrm{TR}_{\beta}\right)$.

4. Model 4: Standard TR model (2.4.1) (FE-TR).

5. Model 5: RE-TR with random intercept on the drift (RE-TR).

6. Model 6: Cox PH Model.

The fitted DPM-TR models follow the model specification in (4.1.2). The regression parameters for the standard TR model were assigned the same priors as $\mathbf{a}$ and $\mathbf{b}$ from the DPM-TR model. In order to establish similar priors between the fitted Random Effect and DPM-TR models, we note that Random Effects models can accurately be understood as limiting cases of corresponding DPM models with $\gamma \rightarrow \infty$. As such, our fitted FE-TR models were assigned a prior on the mixture distribution $G$ equal to the prior assigned to the base measure $H$ in the DPM-TR model. The Cox Model was fit by maximizing the partial-likelihood.

\subsubsection{Results}

Survival function estimation, model selection, and inference/parameter estimation are all integral components for any complete analysis of survival data. The remainder of this section 
is dedicated to exploring results touching on performance of the proposed methodology in each of these areas.

One of the principal benefits of fitting parametric or semi-parametric survival models is the ability to interpolate survival estimation. Such interpolation is not readily possible using nonparametric methods. In that light, we were primarily interested in the accuracy of survival estimation conditional on covariate values $v_{i}$ and $w_{i}$. We evaluated this accuracy by estimating survival conditional on the median value of $v_{i}\left(v_{i}=0\right)$ across all 4 treatment levels for $w_{i} \in W=\{0,0.2,0.4,1\}$ and summarized estimation error using the following function norm:

$$
L_{2}(\widehat{S} \mid v=0)=\left(\sum_{w^{*} \in W} \int_{0}^{t_{\max }}\left(\widehat{S}\left(t \mid v=0, w=w^{*}\right)-S\left(t \mid v=0, w=w^{*}\right)\right)^{2} d t\right)^{1 / 2}
$$

where $t_{\max }$ is an appropriate upper bound on survival time. Although the $L_{2}$ norm is not commonly used to evaluate survival estimation, it was deemed appropriate for our purposes due to its ability to more heavily penalize large departures from the truth across all time points. Because survival estimation is generally poor in the positive tails, we set $t_{\max }$ to be the time at which $95 \%$ of subjects are expected to have experienced the event. As such, $t_{\max }$ was distinct for each of the 5 Data Cases. The $L_{2}$ survival error (4.4.7) was recorded for all fitted models.

Median $L_{2}$ survival errors within Data Case, Fitted Model, and Censoring Level are plotted in Figure 4.1. Several patterns are worth noting. First, and quite unsurprisingly, fitting the correct model generally resulted in optimal or near optimal conditional survival

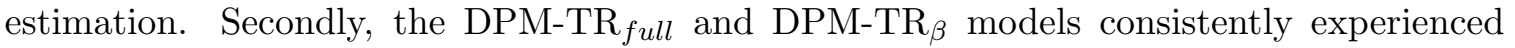
among the least survival estimation error across all Data Cases, even when there was no unmeasured heterogeneity in the data (Data Case 4). The only exception to this pattern was when data were generated from the random effects model (Data Case 5) and censoring was high. In this particular case, the correct RE-TR model displayed substantially better survival estimation. As expected, the standard TR model provided very poor survival estimation whenever there was latent heterogeneity in the data (Cases 1,2,3,5). In fact, the standard TR model performed so poorly in data cases with latent heterogeneity that the 


\section{Median $\mathrm{L}_{2}$ Survival Estimation Error}
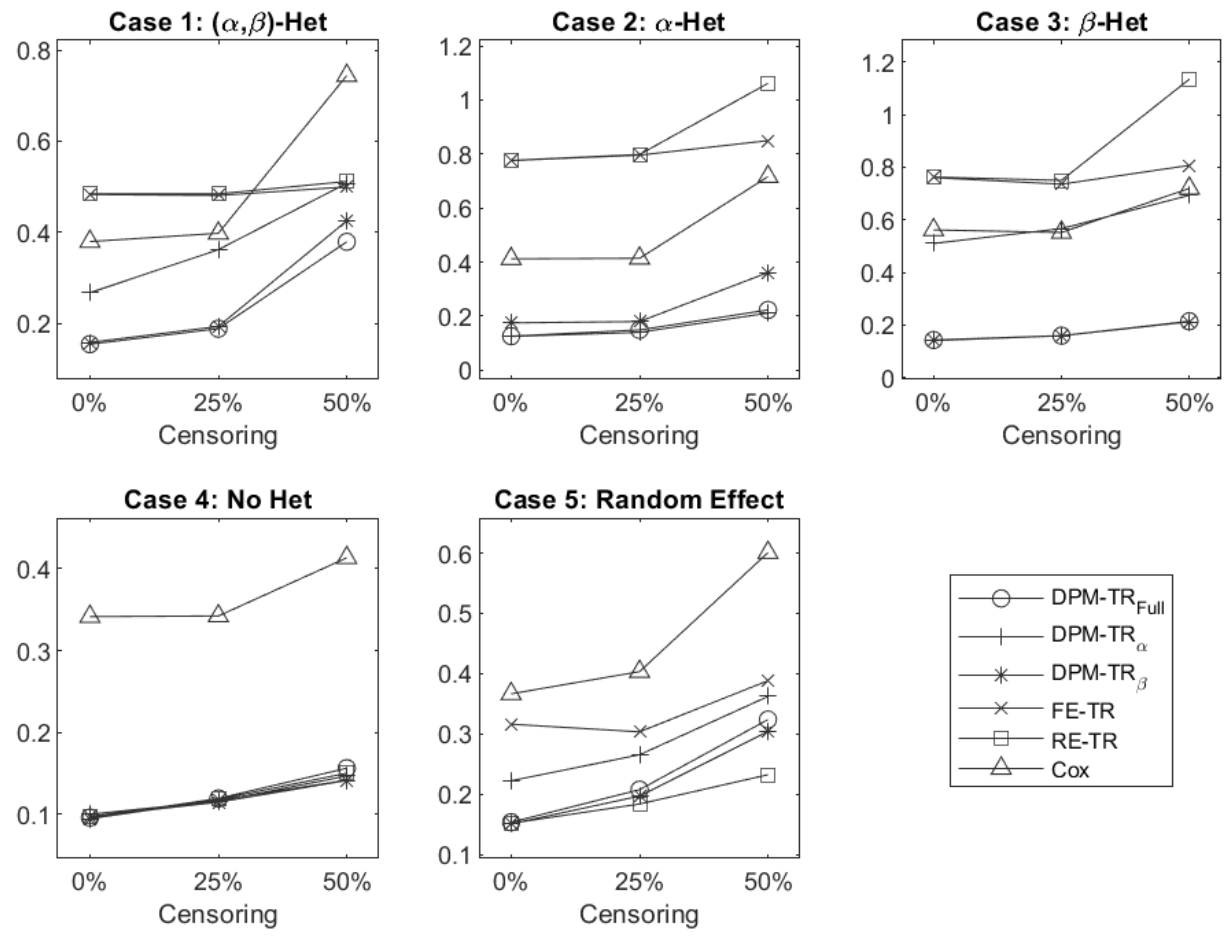

Figure 4.1: Median $\mathcal{L}_{2}$ distance between true and estimated conditional survival functions is displayed for each fitted model across all Case $\times$ Censoring data combinations. 
Cox model often provided superior survival estimates in spite of the fact that the data were generated to explicitly violate the $\mathrm{PH}$ assumption. It is also worth noting that the Random Effects models also demonstrated worse survival estimation than the Cox model when data were generated from a discrete mixture.

We next considered parameter estimation where our primary interest was in estimating covariate effects. Due to the Bayesian nature of the TR based models used in our study, we focused our attention on posterior median estimates of population average regression parameters. Specifically, our principal interest was in the ability to estimate population average coefficient $\alpha_{1}=E\left[\alpha_{i 1}\right]$ and $\beta_{1}=E\left[\beta_{i 1}\right]$. We considered the posterior median as our estimator of interest and used bias, e.g. mean difference from true parameter values, and Root Mean Square Error (RMSE) across simulations to evaluate parameter estimation. Plots of both measures for $\alpha_{1}$ and $\beta_{1}$ can be found in Figures 4.2 and 4.3, respectively. It should be noted that bias is plotted as a proportion of the true value, i.e. $\operatorname{Bias}(\hat{\theta})=\left(\frac{\hat{\theta}-\theta}{\theta}\right)$, so that "positive" values imply bias away from $H_{0}: \theta=0$. RMSE is plotted on the log-scale to account for magnitude difference across fitted models.

Several patterns similar to those previously discussed for survival estimation were also observed for parameter estimation. First, fitting the true model generally resulted in minimal bias and RMSE. When data were generated with no latent heterogeneity (Data Case 4), all fitted models demonstrated fairly accurate estimation, with "simpler" models tending to have lower RMSE. The DPM-TR full model provided fairly unbiased parameter estimation

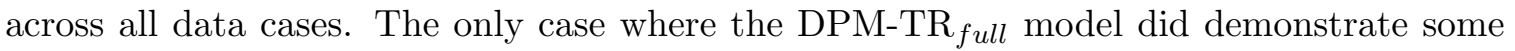
noticeable bias was when data were generated from the Random Effect model (Case 5). Yet even in this case the bias of the DPM-TR full model was towards the null hypothesis so that resulting inference is conservative. The tendency for the DPM-TR full model to provide generally unbiased estimation did come at some cost in terms of estimator variability, though not as much as might be expected given the amount of flexibility afforded by the model. For most cases considered, the RMSE for parameter estimates from the DPM-TR full $_{\text {model }}$ are less than $50 \%$ higher than the RMSE for parameter estimates from the correct model. In many cases the difference is actually much smaller. 


\section{Estimate Bias for $\alpha_{1}$}
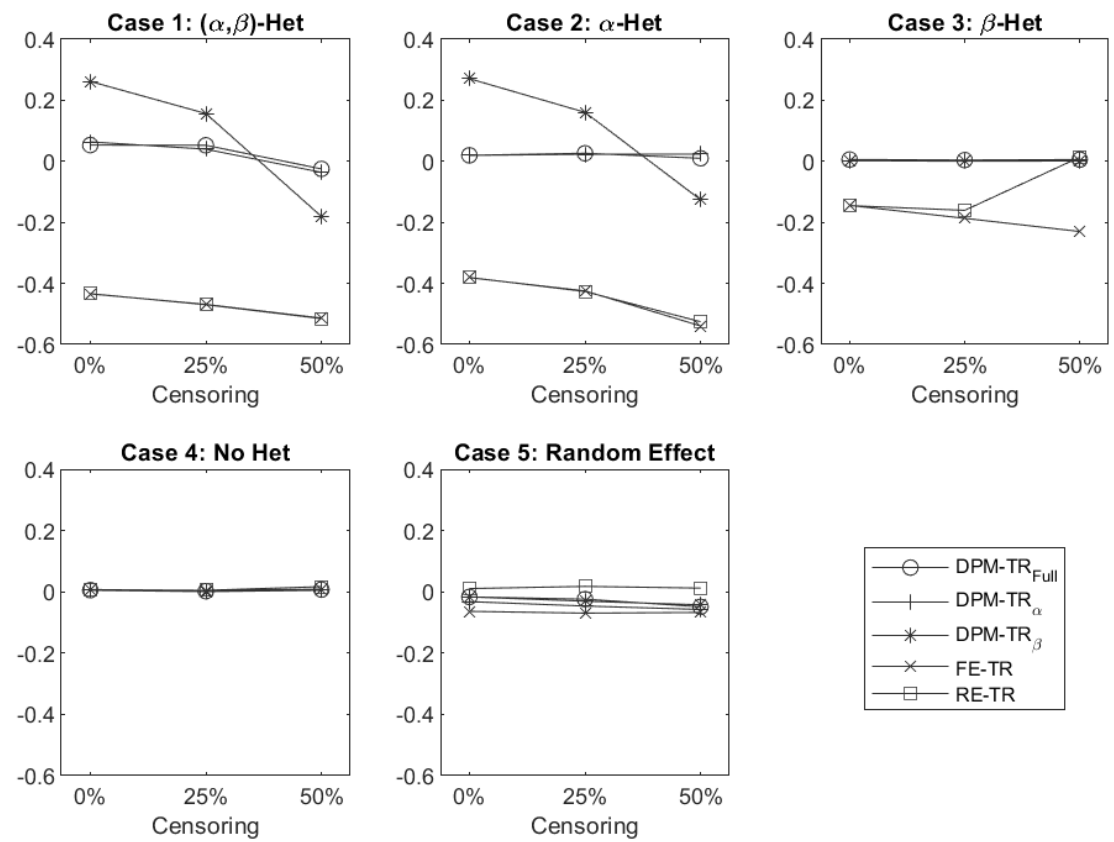

\section{$\log$ RMSE for $\alpha_{1}$}
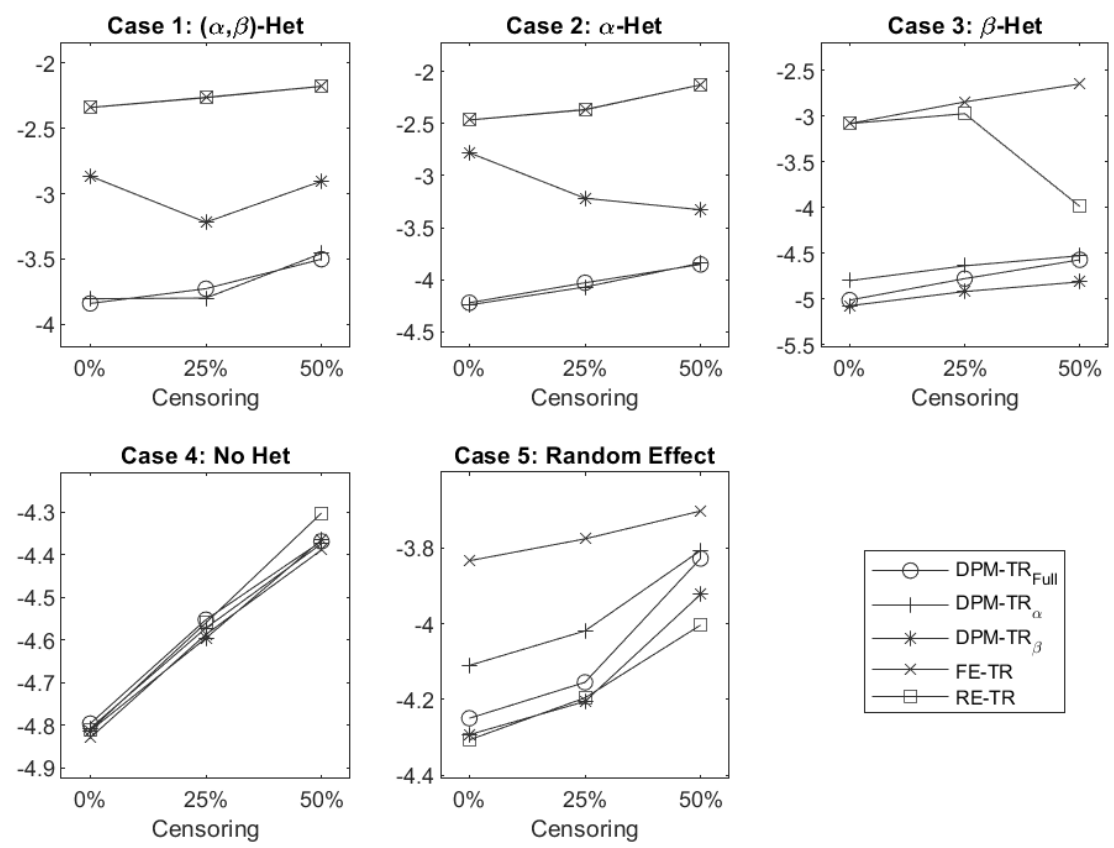

Figure 4.2: Plots of parameter estimate bias and RMSE for $\alpha_{1}$ across three censoring levels and all 5 data cases. Bias is plotted as a proportion of the true parameter value. RMSE is plotted on the log-scale. 


\section{Estimate Bias for $\beta_{1}$}
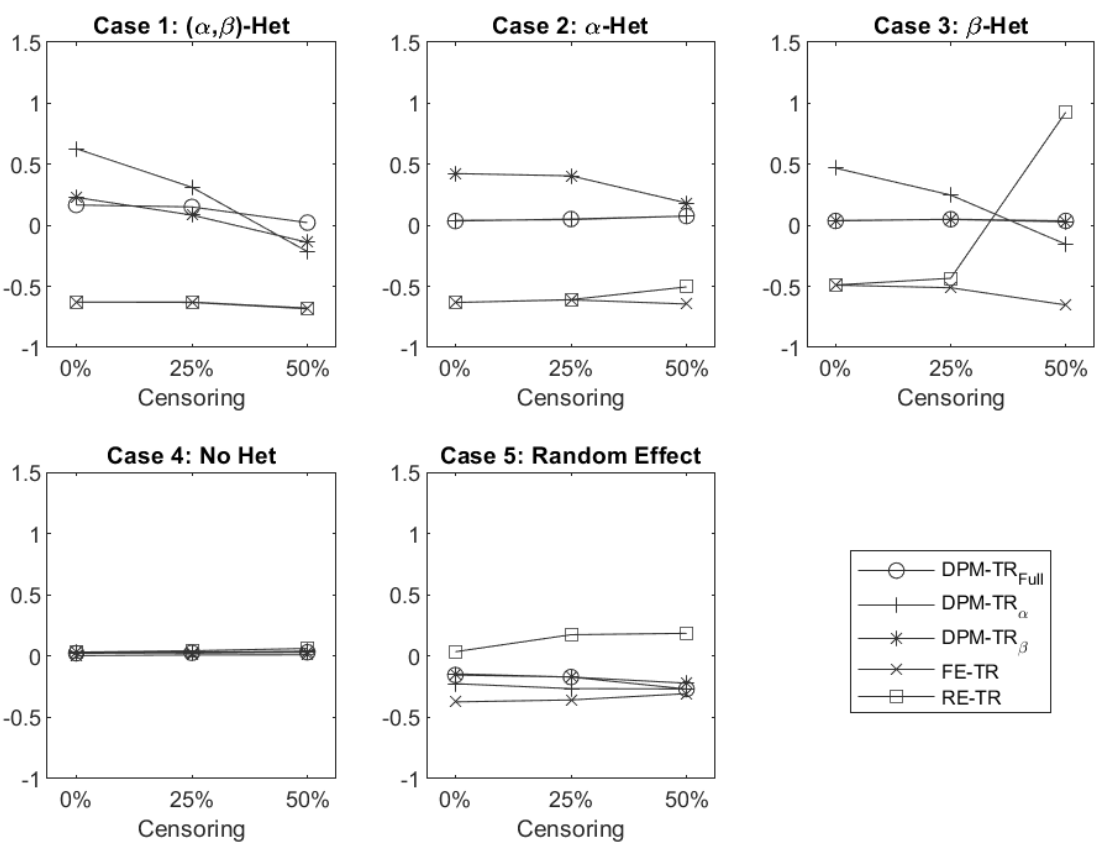

\section{$\log$ RMSE for $\beta_{1}$}
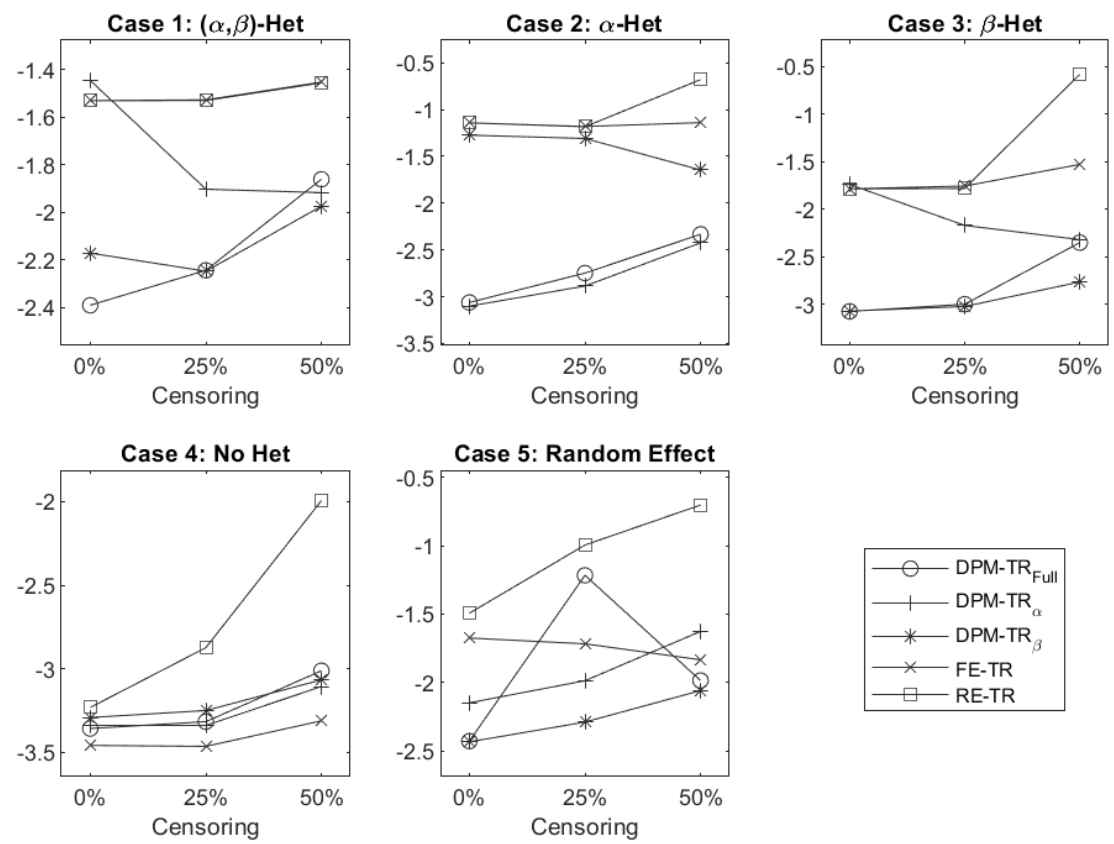

Figure 4.3: Plots of parameter estimate bias and RMSE for $\beta_{1}$ across three censoring levels and all 5 data cases. Bias is plotted as a proportion of the true parameter value. RMSE is plotted on the log-scale. 
In stark contrast to the above observations, the DPM-TR $\alpha$ and DPM-TR $\beta$ models did not provide consistently accurate estimation of regression parameters. Specifically, as can be seen in Figures 4.2 and 4.3, the DPM-TR ${ }_{\alpha}$ model did not provide consistent estimation of $\beta_{1}$ in data cases where heterogeneity existed in the drift regression parameters $\boldsymbol{\beta}$ (Data

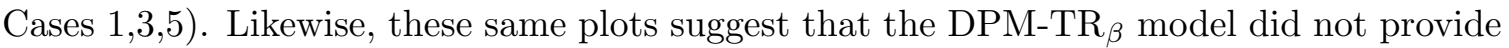
consistent estimation of either parameter in data cases where heterogeneity existed in the initial health regression parameters $\boldsymbol{\alpha}$ (Data Cases 1,2). Note, however, that the DPM-TR $\beta$ model did perform well when data were generated with a normal random effect $\beta_{0 i}$ (Data Case 5). Though not surprising, this result is worth mentioning because the data generating

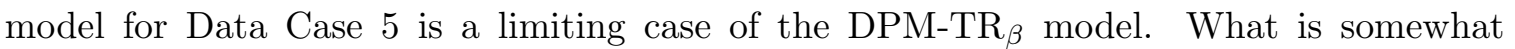
surprising about Data Case 5 is the result that estimates of $\beta_{1}$ from the true random effects model had relatively large RMSE compared to other models. The same pattern occurs in the case where data are generated with no latent heterogeneity (Data Case 4). In both cases we would expect the random effects model to perform at least as well as the other mixture models, especially in light of the fact that the RE-TR model did provide good survival estimation in both cases. It is possible that this poor performance from the RE-TR model in estimation of $\beta_{1}$ is an artifact of the particular regression parameters chosen for our simulation study. Further exploration of this matter would be worthwhile

The final point of interest in our simulation study relates to model selection. Our observations concerning both survival and parameter estimation suggested that, at a minimum, it would be desirable for model selection criteria to be capable of the following:

1. Distinguish when data come from the FE-TR model.

2. Determine whether any latent heterogeneity arises from a continuous mixture (e.g. the RE-TR model) or due to a discrete mixture (e.g. the DPM-TR models).

3. Determine whether latent heterogeneity is located in regression coefficients associated with initial health $x_{0 i}$ or regression coefficients associated with health trend $\mu_{i}$.

In order to understand general patters in the PML criterion performance across Data Cases, we considering median differences in Pseudo-Marginal Likelihood (PML) values between 
each fitted model and the fitted "true" model. Plots of these values are presented in Figure 4.4. We consider model $M_{j}$ to be superior to model $M_{k}$ if $P M L\left(M_{j}\right) / P M L\left(M_{k}\right)>20$ (Kass \& Raftery, 1995); i.e., model $M_{j}$ is considered superior if we estimate that the posterior probability of model $M_{j}$ being correct is 20 times higher than the posterior probability of model $M_{k}$. With this in mind, several features from Figure 4.4 are worth noting. First, the PML criterion was able to identify when the FE-TR model is sufficient (Data Case 4). Second, the PML criteria was largely able to identify when the data come from finite mixtures (Data Cases 1-3). The lone exception came in Data Case 3 with high censoring. In this case censoring was sufficiently high that the RE-TR model was generally able to model the data just as well as the DPM-TR models. In the case where data are generated from the RE-TR model (Data Case 5), the PML did tend to select the current model. It should here be restated that the RE-TR model did not provide ideal parameter estimation even when it was the correct model. That the PML selects the RE-TR in spite of poor parameter estimation reinforces our earlier comment that this poor estimation is likely an artifact of the particular regression parameters chosen to generate the data. Somewhat more importantly, the PML criterion was largely unable to distinguish between potential sources of heterogeneity in the discrete mixture cases (Data Cases 1,2,3). In fact, For Data Cases 2 and 3, the PML tended to select the DPM-TR model with the "opposite" heterogeneity structure from what actually generated the data. This is, of course, not ideal. Taken together, these comments suggest that use of the PML criteria in selecting a heterogeneity structure should be limited to applications where distinguishing between the FE-TR, RE-TR, and DPM-TR full models is sufficient.

In light of the noted weaknesses in the PML criterion, a few comments regarding alternative model selection criteria are appropriate. After noting the surprising patterns in the difference between PML values for the DPM-TR models fit to data generated from finite mixtures, the authors attempted to identify the cause of the noted poor performance. When DPM-TR models with the "wrong" heterogeneity structure were fit, the model identified a very large number of groups without, apparently, sufficiently penalizing this additional complexity. In light of the well-established connection between PML methods and the AIC 
Median PML Differences
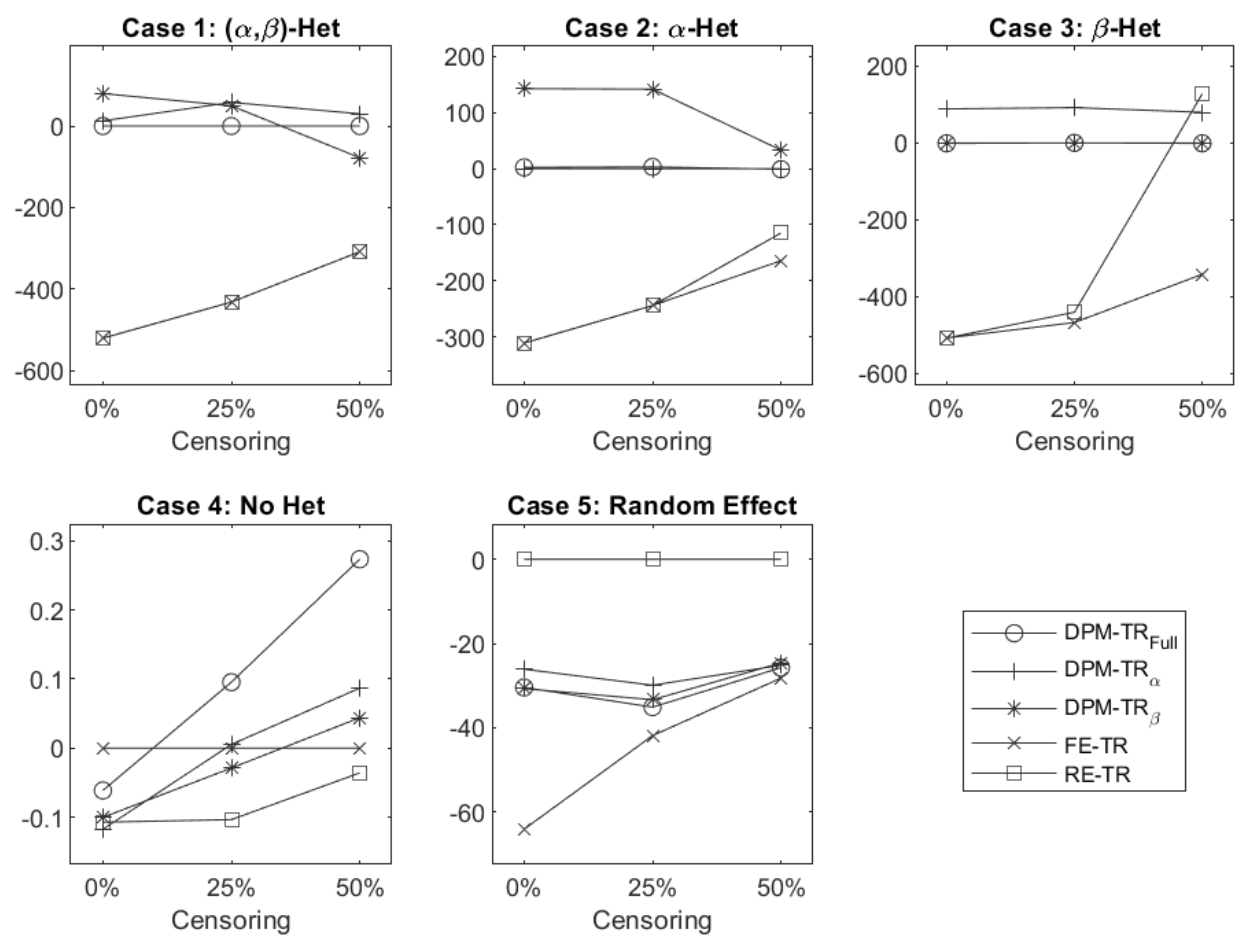

Figure 4.4: Median differences between PML values for each fitted model and the fitted true model.

(Gelfand \& Dey, 1994; Stone, 1977), it is possible that information criteria which more heavily penalize excessive model parameters such as the BIC might be more successful in discriminating between sources of discrete heterogeneity in TR models. How alternative model selection methods might be applicable to the DPM-TR model is an area worth further consideration in future research.

\subsection{Data Example}

We close this chapter by applying the developed methodology to a National Toxicology Program (NTP) dataset (USDHHS, 2004). This study exposed groups of 48 male and 48 female genetically homogeneous mice to drinking water containing combinations of urethane $(0,10,30$, or $90 \mathrm{ppm})$ and ethanol $(0 \%, 2.5 \%, 5 \%)$. Mice were followed until death or 
sacrifice at end of study. Body weight was also taken regularly and, upon death, each animal underwent autopsy to determine the presence of tumors. We will be interested in effects of urethane on overall survival of the male subpopulation independent of ethanol exposure. While the effect of ethanol exposure was originally of interest, past analysis of these data have established that any effects of ethanol on survival are not statistically significant. Thus, in order to simplify our example application, we limit consideration to the effect of urethane.

The randomized design and large sample size of the study implies that a wide variety of survival methods are appropriate to analyze these data. We believe at the outset, however, that the Cox PH model is contraindicated due to likely violations of the $\mathrm{PH}$ assumption. Specifically, we expect that the effects of urethane exposure are likely to accumulate over time, implying that the hazard ratio between treated and untreated subjects should increase as the study progresses. Inverse Gaussian models are conceptually appealing due to their ability to model such patterns in the hazard function by relating covariates to subject specific drift terms $\mu_{i}$. It is common in toxicology studies to model exposure as a fraction of maximum possible dose and we choose to follow this standard. After some exploratory modeling it became apparent that the effect of urethane on drift was not linear and a log-transformation of urethane treatment might be necessary. To accommodate these two modeling goals, we settled on the following regression structure for subject specific drifts:

$$
\mu_{i}=\beta_{0}+\beta_{1} \times \log \left(\frac{\text { urethane }_{i}+1}{\max \left\{\text { urethane }_{+1}+1\right.}\right)
$$

For the sake of example we also wish to include a covariate related to initial health $x_{0 i}$. Baseline weight is a conceptually reasonable covariate to associate with initial health, giving the following regression structure on $x_{0 i}$ :

$$
\log \left(x_{0 i}\right)=\alpha_{0}+\alpha_{1} \times \text { weight }_{i} .
$$

After specification of model regression structures, it is next necessary to give careful consideration to the most likely sources of latent heterogeneity. For example, the study utilized genetically homogeneous mice suggesting the possibility that little heterogeneity 
exists in baseline health. On the other hand, many non-genetic heterogeneities are likely to persist. Differences in feeding and drinking habits between mice could, for example, induce unmeasured heterogeneity in exposure rates. Such heterogeneity could result in distinct patterns of tumor development and health drift. In reality, even though the data come from a well controlled randomized trial, a wide variety of potential heterogeneities are still possible and even likely. If our goal were a genuine analysis utilizing a maximally flexible model, then the DPM-TR full model would seem reasonable. Given, however, that our current purpose relates primarily to offering a real world data example, we will fit models analogous to the six used in the earlier simulation study.

We first consider survival estimation results. In contrast with our preceding simulation study, we now consider survival conditional only on treatment group in order to compare estimates from our model with corresponding KM curves. Plots of survival estimates from several models are presented in Figure 4.5. Severe lack of fit, at least compared to the KM estimates, is clearly visible for the FE-TR and RE-TR models. This lack of fit is largely due to a distinct cluster of early deaths between days 100-200. Moderate lack of fit is also visible for the Cox model, especially in the Urethane $=90$ treatment group. In contrast, the DPM-TR full model demonstrates strong agreement with the KM estimates. We can quantify this agreement between model-derived and KM estimates of treatment specific survival by considering the $L_{2}$ distance between the latter and former. Table 4.1 contains the relevant results. As noted from the previous plots, it is apparent that survival estimates from the DPM-TR full model are most consistent with corresponding Kaplan-Meier curves. The Cox model performs significantly worse across all treatment levels, though this was largely expected given our belief that the PH assumption likely doesn't hold for these data. Although not included here, one can show that the Schoenfeld residuals from the fitted Cox model do in fact demonstrate a statistically significant trend in time, implying violation of proportional hazards.

Parameter estimates, standard errors, CIs, and significance levels for all models can be found in Table 4.2. Several patterns are worth noting. First, all models estimate a statistically significant relationship between urethane exposure and survival. More impor- 


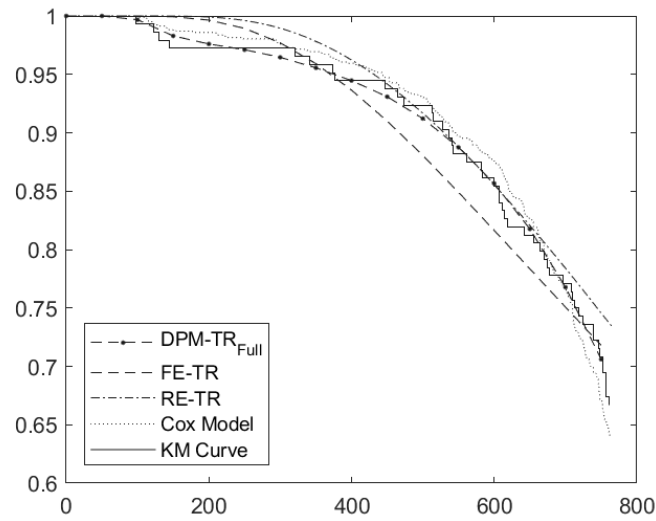

(a) Urethane $=00$

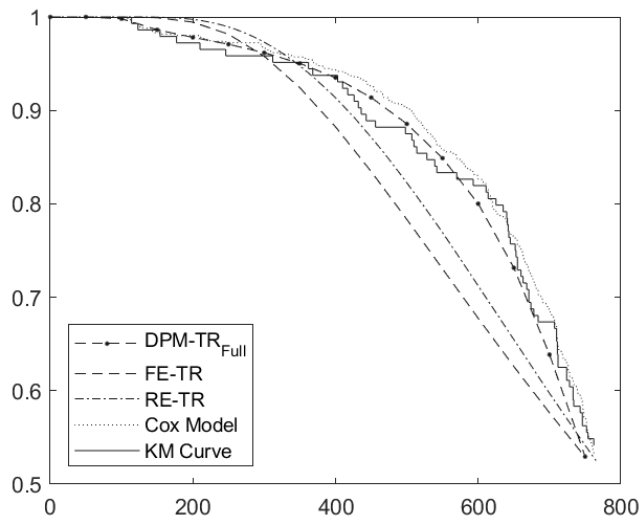

(c) Urethane $=30$

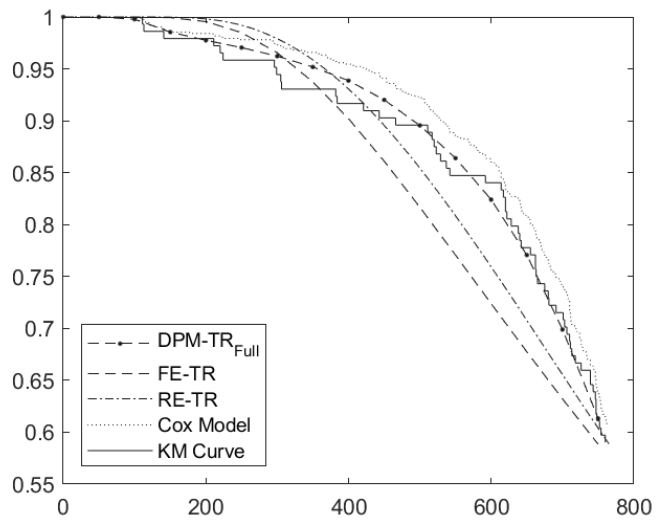

(b) Urethane $=10$

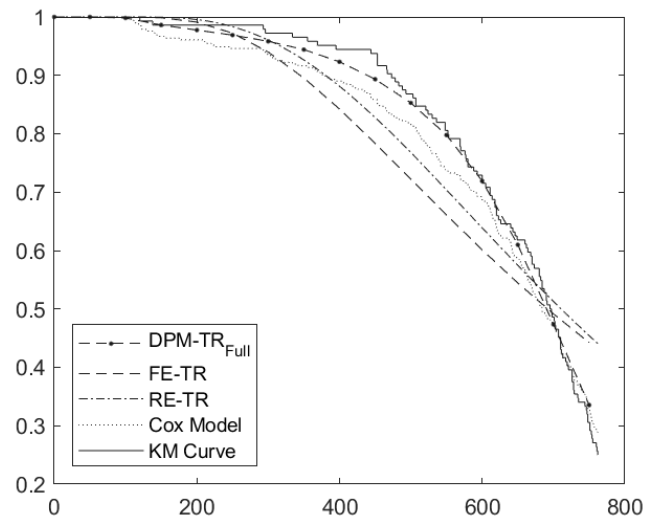

(d) Urethane $=90$

Figure 4.5: Estimated survival (in days) for the NTP dataset conditional on urethane treatment level. Survival estimates are presented for four of the fitted models. It should be noted that estimated survival under the RE-TR Model is visually indistinguishable from those under the standard TR Model. The nonparametric Kaplan-Meier estimates are also included for reference.

\begin{tabular}{|l|llllll|}
\hline & DPM-TR $_{\text {full }}$ & DPM-TR $_{\alpha}$ & DPM-TR $_{\beta}$ & FE-TR & RE-TR & Cox \\
\hline Marginal & 0.146 & 0.115 & 0.207 & 1.489 & 1.501 & 0.200 \\
Urethane $=00$ & 0.188 & 0.390 & 0.207 & 0.644 & 0.663 & 0.653 \\
Urethane $=10$ & 0.351 & 0.516 & 0.410 & 1.528 & 1.549 & 0.479 \\
Urethane $=30$ & 0.453 & 0.737 & 0.568 & 1.914 & 1.919 & 0.858 \\
Urethane $=90$ & 0.508 & 0.755 & 0.589 & 2.427 & 2.429 & 1.060 \\
\hline
\end{tabular}

Table 4.1: $\mathcal{L}_{2}$ distance between survival function estimates from various DPM-TR models and the Kaplan-Meier estimates. 


\begin{tabular}{|c|l|llllll|}
\hline \multicolumn{2}{|c|}{} & DPM-TR $_{\text {full }}$ & DPM-TR $_{\alpha}$ & DPM-TR $_{\beta}$ & FE-TR & RE-TR & Cox \\
\hline$\alpha_{0}$ & Est & 5.123 & 5.136 & 4.966 & 3.908 & 3.911 & - \\
& UB & 5.637 & 5.551 & 5.338 & 3.987 & 3.987 & - \\
& LB & 4.775 & 4.760 & 4.602 & 3.828 & 3.829 & - \\
& SE & 0.214 & 0.194 & 0.205 & 0.040 & 0.041 & - \\
& P & $<0.001$ & $<0.001$ & $<0.001$ & $<0.001$ & $<0.001$ & - \\
\hline \multirow{2}{*}{$\alpha_{1}$} & Est & -0.015 & -0.015 & -0.008 & -0.032 & -0.032 & 0.058 \\
& UB & -0.001 & -0.001 & 0.005 & -0.014 & -0.014 & 0.106 \\
& LB & -0.036 & -0.034 & -0.023 & -0.049 & -0.049 & 0.011 \\
& SE & 0.009 & 0.008 & 0.007 & 0.009 & 0.009 & 0.024 \\
& P & 0.029 & 0.014 & 0.111 & $<0.001$ & $<0.001$ & 0.008 \\
\hline$\beta_{0}$ & Est & -0.266 & -0.259 & -0.234 & -0.062 & -0.062 & - \\
& UB & -0.180 & -0.173 & -0.151 & -0.053 & -0.053 & - \\
& LB & -0.439 & -0.383 & -0.343 & -0.070 & -0.070 & - \\
& SE & 0.065 & 0.050 & 0.054 & 0.004 & 0.004 & - \\
& P & $<0.001$ & $<0.001$ & $<0.001$ & $<0.001$ & $<0.001$ & - \\
\hline$\beta_{1}$ & Est & -0.025 & -0.012 & -0.022 & -0.006 & -0.006 & 0.249 \\
& UB & -0.008 & -0.008 & -0.006 & -0.004 & -0.004 & 0.330 \\
& LB & -0.071 & -0.018 & -0.065 & -0.008 & -0.008 & 0.169 \\
& SE & 0.016 & 0.003 & 0.016 & 0.001 & 0.001 & 0.041 \\
& P & $<0.001$ & $<0.001$ & 0.001 & $<0.001$ & $<0.001$ & $<0.001$ \\
\hline
\end{tabular}

Table 4.2: Parameter estimates for models fit to the NTP dataset. For TR based models we include parameter estimates in the form of posterior medians, posterior standard errors, upper (UB) and lower (LB) 95\% credible interval bounds, and 2-sided significance. Corresponding Cox model parameters, estimated using the standard pseudo-MLE method, are included for comparison. It is worth noting that the Cox model has comparable conclusions to the TR models; i.e., the Cox model concludes that increased weight and increased dose are associated with increased hazard.

tantly, all 6 models agree that an increase in exposure is associated with a decrease in expected survival (recall that $\beta_{1}>0$ implies an increased hazard under the Cox model where $\beta_{1}<0$ indicates a larger hazard under the TR model). All TR based models except for the DPM-TR $\beta$ estimate a statistically significant decrease in survival for increased birth weight. The Cox model again agrees and estimates that weight is associated with increased hazard 


\subsection{Discussion}

Threshold Regression is a conceptually appealing methodology for analyzing time-to-event data thought to arise from developments in an underlying latent process. However, as is often the case in regression models with strong distributional assumptions, both survival function estimation and related inference in TR can suffer in the presence of model misspecifications such as those caused by latent subject heterogeneity. In this chapter we addressed issues related to model inflexibility by applying a tractable and well-studied Bayesian mixture methodology to the TR model. Our simulation study and data example demonstrated that the proposed DPM-TR model provides survival estimation which is comparable to nonparametric methods in terms of flexibility. Additionally, the proposed methodology has the added benefit of being able to interpolate survival between treatment groups; an ability standard nonparametric methods lack.

In spite of these positive results, the current work has several notable limitations. Perhaps most significantly, it is unclear what impact violations of underlying FHT assumptions has on our methodology's performance. By this we mean that TR, and by extension the proposed DPM-TR model, are predicated on the assumption that event times arise as a result of developments in a latent continuous process with independent increments of constant mean and variance. Little research has been undertaken to explore the extent to which various violations of the latent process model might cause issues with inference. In the only paper of which the author is aware that explores such issues, Stogiannis and Caroni (2013) found that application of TR model to data generated from the Weibull distribution resulted in substantially decreased power compared to fitting the correct Weibull model. It is not intuitively obvious to what extent, if any, the proposed mixture model might mitigate potential issues with inference due to inappropriate assumptions about the latent process. The other major limitation of the current work relates to performances of the PML for model selection purposes. While the PML was able to discriminate between discrete and continuous mixture models in our simulation, it was not able to determine whether heterogeneity originated in the initial health $x_{0 i}$ or the drift $\mu_{i}$. The reason for this failure is 
likely the fact that the PML is a measure of global fit and the DPM-TR model is sufficiently flexible to compensate for misspecification of the heterogeneity structure. Identifying an alternative model selection method capable of the desired discrimination capability would be clinically useful. Such a method would likely need to penalize model complexity more than the PML method explored which is closely related to the AIC. Such a method would need to be capable of isolating fit of the different model components, but this may be difficult due to potential identifiability issues between the initial state $x_{0 i}$ and drift $\mu_{i}$. Our suggestion is that the BIC be explored next, though it is not intuitively obvious how the BIC might be calculated for DPM models.

Until a more robust model selection method is available we recommend using the DPM-TR $_{\text {full }}$ model for TR analyses where the FE-TR and RE-TR model provide insufficient flexibility. Our justification for this recommendation rests on the recognition that the $\mathrm{DPM}_{-} \mathrm{TR}_{\text {full }}$ model displayed unbiased parameter estimation and accurate survival estimation even when data were generated with less heterogeneity than the DPM-TR full $_{\text {model }}$

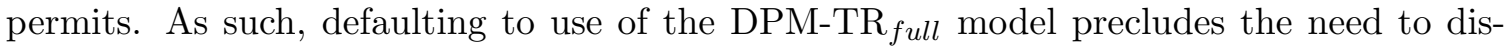
criminate between models specifying different heterogeneity structures while still providing accurate estimation. We also noted, however, that the flexibility of the DPM-TR full comes as the cost of increased estimator variability so that the less flexible FE-TR and RE-TR models will generally provide higher inferential power when they are appropriate.

In addition to the need for supplemental work exploring performance of the DPMTR model, the proposed methodology opens several avenues for further methodological refinement. The TR paradigm has been utilized in several unique contexts such as analysis of recurrent events; e.g., Economou et al. (2015). The added flexibility of the DPM-TR model is likely to extend the applicability of such methods. Other work has extended the TR model to incorporate knowledge of observable processes which might be related to event occurrence; e.g., G. Whitmore, Crowder, and Lawless (1998). Such methods are currently limited by the fairly strong assumption that the latent and observable processes jointly behave as a bivariate Wiener diffusion. Such an assumption is likely to be problematic in many potential applications and implementation of DP Mixture priors in marker process 
settings might greatly extend the usefulness of these models. 


\section{Chapter 5 \\ Testing for Ordinal Treatment EFFECTS on Survival Via Dynamic Mixture of Dirichlet Processes FOR THRESHOLD REgRESSION}

Sometimes one creates a dynamic impression by saying something, and sometimes one creates as significant an impression by remaining silent.

- The Dalai Lama

Ordinal treatment data can be found in a variety of biomedical settings ranging from dose-finding experiments in pharmaceutical development to toxicology studies in animals. While ordinal treatment data can be analyzed using tools commonly employed in the more general case of categorical treatments, explicit modeling of the ordinality structure can often be exploited to produce more powerful analysis tools. Most current methods to analyze the effect of an ordinal treatment on survival are related to the Tarone-Ware trend test (Tarone \& Ware, 1977) which is a weighted logrank test with an ordered alternative. Such methods have several weaknesses. First the Tarone-Ware test can be highly insensitive to scale shifts due to treatment level. By this we mean that when the median survival times between treatment groups are similar, the Tarone-Ware test is often not powerful in detecting differences in tail behavior of the event time distributions. The second major issue with Tarone-Ware based tests relates to possible methods to adjust for potential confounding. As a logrank based test, the Tarone-Ware model can only adjust for discrete or 
discretized confounders via stratification. The inability to adjust for continuous confounders can be problematic in observational studies and other settings outside of controlled trials. In the current chapter we propose a flexible model based on the Dynamic Mixture of Dirichlet Processes model proposed by Pennell and Dunson (2008). Our proposed model, closely related to the DPM-TR model presented in Chapter 4, is capable of testing for effects of an ordinal treatment on time-to-event without the limitations just discussed. In contrast with the DPM-TR model, though, the model proposed in the current chapter explicitly models expected dependence between treatment groups. This structure results in a model which is sensitive to both location shifts and shape changes in survival and can adjust for both continuous and discrete confounders. In order to develop an adequate understanding of our proposed methodology, we begin by presenting a general review of the Dynamic Mixture of Dirichlet Processes (DMDP) model. We then apply the DMDP methodology to the TR context which has been the foundation of this entire work. We conclude by presenting results from a simulation study and data example which contrasts the proposed method with alternative ordinal treatment survival methods currently available.

\subsection{General DMDP Framework}

Consider a response vector $\mathbf{Y}_{h i}$ measured on subject $i\left(i=1, \ldots, n_{h}\right)$ in treatment group $h(h=1, \ldots, d)$ such that group-specific treatment levels $\left(X_{1}, \ldots, X_{d}\right)$ have some natural ordering $X_{1}<X_{2}<\ldots, X_{d}$. We call data with such a structure ordinal treatment data. It is quite natural to conceptualize such data as coming from a mixture model with treatment specific mixture distributions, i.e.

$$
\begin{aligned}
, \mathbf{Y}_{h i} & \sim F\left(\mathbf{y} \mid \boldsymbol{\theta}_{h i}\right), \\
\boldsymbol{\theta}_{h i} & \sim G_{h}
\end{aligned}
$$

where the $G_{h}$ are unknown. Model 5.1.1 makes the intuitive assumption that differences in the outcome distributions, $F_{h}$, between treatment levels are actually due to differences in the distributions of the parameters $\boldsymbol{\theta}_{h i}, G_{h}$, which give rise to the observed data. Testing 
for differences between adjacent treatment levels $h$ and $h+1$ can, in this context, be reinterpreted as testing for differences in the mixture distributions $G_{h}$ and $G_{h+1}$.

It is important here to note that Model 5.1.1 is actually sufficiently general to apply well beyond the ordinal treatment setting. By defining mixtures distributions $G_{h}$ as being unique to treatment group $h$, we have explicitly modeled a basic assumption found in most categorical treatment data; subjects in the same treatment group are expected to be more similar than subjects in different treatments. In the context of ordinal treatment data, however, we would generally expect a stronger behavior than this. Specifically, we will often expect ordinal treatment data to display a proximity dependence where subjects in adjacent treatments are more likely to be similar than subjects in more distant treatment groups. Consider, as an example, the NTP study explored in the previous chapter. In that study mice were exposed to different levels of urethane and followed for tumor development and overall survival. We would expect outcomes in the highest treatment group to be more similar to those for the intermediate treatment groups than with outcomes for the control. In fact, such a pattern is quite apparent in Figure 4.5 where each increase in urethane treatment results in decreased survival. One method of capturing such dependence assumes that moving from treatment $h$ to $h+1$ results in an "innovation" for the mixing distribution $G_{h+1}$ compared to $G_{h}$. We can express this notion symbolically as:

$$
\begin{aligned}
\mathbf{Y}_{h i} & \sim F\left(\mathbf{y} \mid \boldsymbol{\theta}_{h i}\right), \\
\boldsymbol{\theta}_{h i} & \sim G_{h}, \\
G_{h} & = \begin{cases}G_{1}^{*}, & \text { if } h=1, \\
\left(1-\pi_{h}\right) G_{h-1}+\pi_{h} G_{h}^{*}, & \text { if } h=2, \ldots, d,\end{cases}
\end{aligned}
$$

where $G_{h}^{*}$ is called the innovation distribution. We can interpret this model as claiming that, for the transition from treatment $h-1$ to $h$, a proportion $\pi_{h} \in[0,1]$ of subjects will have a "new reaction" $G_{h}^{*}$ to the increase in treatment while the remaining $1-\pi_{h}$ proportion of subjects will have the same response for both treatment levels.

While it is possible to specify Model 5.1 .2 with parametric innovation distributions, a 
more flexible solution would be to model the $G_{h}^{*}$ as draws from the Dirichlet Process. Doing so results in a model known in the literature as the Dynamic Mixture of Dirichlet Processes (DMDP) model:

$$
\begin{aligned}
\mathbf{Y}_{h i} & \sim F\left(\mathbf{y} \mid \boldsymbol{\theta}_{h i}\right), \\
\boldsymbol{\theta}_{h i} & \sim G_{h}, \\
G_{h} & =\left(1-\pi_{h}\right) G_{h-1}+\pi_{h} G_{h}^{*}, \\
G_{h}^{*} & \sim D P\left(\gamma_{h} G_{0}\right) .
\end{aligned}
$$

While the mixture in (5.1.3) is obviously capable of modeling complex innovations between adjacent treatment levels due to the flexibility of $G_{h}^{*}$, it should be noted that several simple innovations are also possible. Take for example, the case where a simple location shift occurs in the parameter of interest between mixtures $G_{h}$ and $G_{h-1}$. Such a location shift innovation seems plausible in a variety of situations and can be captured by the model with $\pi_{h}=1$ and $G_{h}^{*}$ being a point mass at the value of the location shift. As another example, scale shifts in the parameter can be modeled with $G_{h}^{*}$ being symmetric about 0 . In truth the model of mixture innovations in (5.1.3) can accommodate a wide variety of simple and complex innovations in model parameters between treatment levels.

Model 5.1.3 assuming a multivariate Normal data distribution $F$ was originally proposed by Pennell and Dunson (2008). The current chapter is dedicated to applying the DMDP methodology to time-to-event data via a First Hitting Time model. Before we do so, however, it will be enlightening to discuss several features of the DMDP independent of any assumptions on $F$. This discussion will occupy the next several sections.

\subsubsection{Hypothesis Testing Under the DMDP Model}

The DMDP model is conceptually appealing as a method for analysis of ordinal treatment data for several reasons. First, we expect this model to display flexibility in the presence of parameter heterogeneity similar to that found in the standard DPM model. Secondly, and somewhat more importantly, the DMDP model easily facilitates testing of a number of 
hypotheses natural to the ordinal treatment setting, including:

1. Local null hypotheses

$$
H_{0}: G_{j}=G_{k}, j \neq k
$$

2. Global null hypothesis

$$
H_{0}: G_{1}=\ldots=G_{d}
$$

Some care must be taken formulating the above hypotheses in the context of the DMDP model, particularly in light of the presence of multiple comparisons which is inherent in the ordinal treatment settings. What follows is a review of the hypothesis formulation scheme proposed by Pennell and Dunson (2008).

The first issue we must address relating to hypothesis testing under the DMDP model is the manner in which we interpret equivalence between treatment-specific mixture distributions $G_{h}$ and $G_{j}$. Such a comparison is naturally facilitated by specifying an appropriate distance metric on the relevant probability space. As an example, the well-known total variation distance will be particularly useful for our purposes.

Definition 5.1.1. Let $G_{h}$ and $G_{j}$ be probability measures on $\mathbb{R}^{q}$ and let $B$ be a Borel set in the Borel $\sigma$-algebra $\mathcal{B}$ on $\mathbb{R}^{q}$. Then the total variation distance between $G_{h}$ and $G_{j}$ is given by

$$
\left\|G_{h}-G_{j}\right\|_{T V}=\max _{B \in \mathcal{B}}\left|G_{h}(B)-G_{j}(B)\right|
$$

Colloquially, the total variation distance is the maximum difference in probability assigned to an event by the two distributions $G_{h}$ and $G_{j}$.

The chief benefit of the $T V$ distance in our current application is the fact that the ordering structure implied by Model 5.1.3 is maintained in terms of Total Variation. The present concern relates to the case where a "non-adjacent" treatment group $h+2$ has outcomes more similar to treatment group $h$ than the adjacent treatment group $h+1$. In such a case we would expect that the mixture distribution $G_{h+2}$ would also be "closer" to 
$G_{h}$ than the mixture distribution $G_{h+1}$, at least in terms of an $L_{p}$ distance; i.e., $\| G_{h+2}-$ $G_{h}\left\|_{p} \leq\right\| G_{h+1}-G_{h} \|_{p}$. Assuming such differences are significant, though, we would still expect the similarity between $G_{h+2}$ and $G_{h}$ to be due to two innovations, $G_{h} \rightarrow G_{h+1}$ and $G_{h+1} \rightarrow G_{h+2}$, instead of there being no innovation between the two treatment levels. Stated another way, we wish our model to allow for later increases in treatment to counteract but not eliminate effects of earlier increases in treatment level. Fortunately, as stated earlier, it can be shown that this type of ordering in the DMDP model is maintained in terms of TV distance. The following theorem establishes the relevant result.

Theorem 5.1.1. Let $G_{h}, G_{h+1}$, and $G_{h+m+1}$ be distributed according to (5.1.3) with continuous base measure $G_{0}$. Then

$$
P\left(\left\|G_{h+m+1}-G_{h}\right\|_{T V} \geq\left\|G_{h+1}-G_{h}\right\|_{T V}\right)=1 .
$$

Proof. Let $\pi_{1}=1$. From the recursive definition of $G_{h}$ we have the following for $h=2, \ldots, \delta$ :

$$
\begin{aligned}
G_{h}(\cdot) & =\left(1-\pi_{h}\right) G_{h-1}(\cdot)+\pi_{h} G_{h}^{*}(\cdot) \\
& =\pi_{h} G_{h}^{*}(\cdot)+\sum_{j-1}^{h-1}\left\{\prod_{m=j+1}^{h}\left(1-\pi_{m}\right) \pi_{j} G_{j}^{*}(\cdot)\right\} \\
& =\sum_{j=1}^{h} w_{h j} G_{j}^{*}(\cdot) \\
& =\sum_{j=1}^{h} w_{h j}\left(\sum_{k=1}^{\infty} p_{j k} \delta_{\theta_{j k}^{*}}(\cdot)\right) \\
& =\sum_{j=1}^{h} \sum_{k=1}^{\infty}\left(w_{h j} p_{j k}\right) \delta_{\theta_{j k}^{*}}(\cdot) .
\end{aligned}
$$

Because the atoms $\theta_{j k}^{*}$ are drawn from a continuous distribution $G_{0}$ we have

$$
P\left(\left\{\theta_{j k}^{*}: j \leq h, k \geq 1\right\} \cap\left\{\theta_{j k}^{*}: j>h, k \geq 1\right\}=\emptyset\right)=1,
$$

i.e. the intersections of the sets of atoms defining each innovation distribution $G_{h}^{*}$ are empty almost surely. As such, the absolute difference in probability assigned by measures $G_{h}$ and 
$G_{h+m+1}$ to an event $B \subseteq\left\{\theta_{j k}^{*}: j=1, \ldots, h+m+1\right\}$ is equal to

$$
\begin{aligned}
\left|G_{h}(B)-G_{h+m+1}(B)\right| & =\left|\sum_{j=1}^{h+m+1} \sum_{k=1}^{\infty} p_{j k} \cdot\left(w_{h j}-w_{(h+m+1) j}\right) I\left\{\theta_{j k}^{*} \in B\right\}\right| \\
& =\left|\sum_{j=1}^{h+m+1}\left(w_{h j}-w_{(h+m+1) j}\right) \sum_{k=1}^{\infty} p_{j k} I\left\{\theta_{j k}^{*} \in B\right\}\right|
\end{aligned}
$$

where $w_{h j}=0$ if $j>h$. The above expression can obviously be maximized by allowing $B$ to be either the set of events where $w_{h j}-w_{(h+m+1) j}>0$ or the set of events where $w_{h j}-w_{(h+m+1) j}<0$. The former case corresponds to the event that $\theta$ belongs to the domain of $G_{h}$, i.e., $B=B_{1}=\left\{\theta_{j k}^{*}: j=1, \ldots, h\right\}$. The latter case corresponds to the event that $\theta$ belongs to the domain of $G_{h+m+1}$ but does not belong to the domain of $G_{h}$, i.e. $B=B_{2}=\left\{\theta_{j k}^{*}: j>h\right\}$. Because $B_{1}$ and $B_{2}$ are disjoint and their union covers the domains of both $G_{h}$ and $G_{h+m+1}$, we have the following:

$$
\begin{array}{r}
G_{h}\left(B_{1}\right)+G_{h}\left(B_{2}\right)=1=G_{h+m+1}\left(B_{1}\right)+G_{h+m+1}\left(B_{2}\right) \\
\Leftrightarrow G_{h}\left(B_{1}\right)-G_{h+m+1}\left(B_{1}\right)=G_{h+m+1}\left(B_{2}\right)-G_{h}\left(B_{2}\right) \\
\Leftrightarrow\left|G_{h}\left(B_{1}\right)-G_{h+m+1}\left(B_{1}\right)\right|=\left|G_{h}\left(B_{2}\right)-G_{h+m+1}\left(B_{2}\right)\right| .
\end{array}
$$

The above implies that $B_{1}$ and $B_{2}$ give the same value for $\left|G_{h}(\cdot)-G_{h+m+1}(\cdot)\right|$. The below expressions for total variation distance follow immediately from letting $B=B_{2}$ in (5.1.9):

$$
\begin{aligned}
\left\|G_{h+1}-G_{h}\right\|_{T V} & =w_{(h+1)(h+1)}=\pi_{h+1}, \\
\left\|G_{h+m+1}-G_{h}\right\|_{T V} & =\sum_{j=h+1}^{h+m+1} w_{(h+m+1) j} .
\end{aligned}
$$

Because the $w$.. values are strictly nonnegative we have

$$
\left\|G_{h+m+1}-G_{h}\right\|_{T V}-\left\|G_{h+1}-G_{h}\right\|_{T V}=\sum_{j=h+2}^{h+m+1} w_{j h} \geq 0,
$$

which established to desired inequality.

The preceding comments demonstrate that a special type of ordering between mixture distributions $G_{h}$ for the DMDP model is maintained in terms of the TV distance. It is worth 
mentioning that alternative method of imposing ordering in the context of the DMDP model was explored by Dunson and Peddada (2008). It is now our goal, though, to restate the hypotheses of interest mentioned at the beginning of this section in terms of the TV distance. Before we can do so, however, we will need to define what we mean for two distributions to be "equal". It is often natural to define equivalence between two objects in the strong sense that there is zero distance between them, i.e. $\left\|G_{h}-G_{j}\right\|_{T V}=0$. It has been argued, however, that null hypotheses specifying such exact matching of distributions are artificial and problematic for inference (Berger \& Delampady, 1987; Nickerson, 2000). Alternatively, and somewhat more pragmatically, we might consider distributions $G_{h}$ and $G_{j}$ equivalent if they "effectively match", i.e. $\left\|G_{h}-G_{j}\right\|_{T V} \leq \epsilon$ for $\epsilon$ small. Effectively matching null hypotheses were employed by Pennell and Dunson (2008) when they generalized the original specification of the DMDP model from Dunson (2006) to allow for testing for differences between treatment levels. As an example, we can restate the null hypotheses mentioned at the beginning of this section in terms of effective matching as follows:

1. Local null hypotheses

$$
H_{0}:\left\|G_{h}-G_{j}\right\|_{T V} \leq \epsilon
$$

2. Global null hypothesis

$$
H_{0}: \forall h \neq j,\left\|G_{h}-G_{j}\right\|_{T V} \leq \epsilon
$$

Such hypotheses are particularly appropriate in the context of the DMDP model because, as will be shown in the next section, they can be simplified to statements about an upper bound on the innovation probability $\pi_{h}$. Due to this intuitive interpretation of model hypotheses, we also employ effective matching null hypotheses throughout our discussion of the general DMDP and in our development of our methodology for time-to-event data. 


\subsubsection{Prior Specifications for the DMDP Model}

We have, to this point, shown that the DMDP model with DP innovation distributions and effective matching null hypotheses maintains the natural ordering we desire in ordinal treatment models. We now turn our attention to controlling the prior probability allocated to local hypotheses concerning adjacent treatments, i.e. $H_{0 h}:\left\|G_{h}-G_{h-1}\right\|_{T V} \leq \epsilon$. Given the recursive definition of $G_{h}$ in (5.1.3), it should seem intuitive that the prior probability placed on $H_{0 h}$ can be controlled by specifying appropriate priors for the innovation probabilities $\pi_{h}$ and the innovation distributions $G_{h}^{*}$. It turns out that $H_{0 h}$ can be rewritten to separate the contributions of these two parameters into distinct components:

$$
\begin{aligned}
H_{0 h}:\left\|G_{h}-G_{h-1}\right\|_{T V} & =\max _{B \in \mathcal{B}}\left|G_{h}(B)-G_{h-1}(B)\right| \\
& =\max _{B \in \mathcal{B}}\left|\pi_{h} G_{h}^{*}(B)+\left(1-\pi_{h}\right) G_{h-1}(B)-G_{h-1}(B)\right| \\
& =\pi_{h}\left\|G_{h}^{*}-G_{h-1}\right\|_{T V} \leq \epsilon .
\end{aligned}
$$

Rewriting $H_{0 h}$ as in (5.1.15) is particularly helpful because it can be shown that $\| G_{h}^{*}-$ $G_{h-1} \|_{T V}=1$ almost surely, implying that the prior weight on $H_{0 h}$ is completely controlled by the prior on the innovation probability $\pi_{h}$.

Theorem 5.1.2. Let $G_{h}, h=1, \ldots, d$, be as in (5.1.3) with continuous base measure $G_{0}$. Then

$$
\left\|G_{h}^{*}-G_{h-1}\right\|_{T V}=1 .
$$

Proof. From (5.1.7), we see that $G_{h-1}$ and $G_{h}^{*}$ assign positive mass to sets of atoms $\boldsymbol{\theta}_{1, h-1}^{*}=$ $\left\{\theta_{j k}^{*}\right\}_{j=1, \ldots, h-1 ; k=1, \ldots, \infty}$ and $\boldsymbol{\theta}_{2, h}^{*}=\left\{\theta_{h k}^{*}\right\}_{k=1, \ldots, \infty}$, respectively. Because $\boldsymbol{\theta}_{1, h-1}^{*}$ and $\boldsymbol{\theta}_{2, h}^{*}$ are countably infinite samples from a continuous distribution $G_{0}$, both have measure 0 with respect to the Lebesgue measure. This implies that $P\left(\boldsymbol{\theta}_{1, h-1}^{*} \cap \boldsymbol{\theta}_{2, h}^{*}=\emptyset\right)=1$. Suppose we choose the Borel set $B=\boldsymbol{\theta}_{1, h-1}^{*}$ or $B=\boldsymbol{\theta}_{2, h}^{*}$, then $\left|G_{h}^{*}(B)-G_{h-1}(B)\right|=1$, and thus $\left\|G_{h}^{*}-G_{h-1}\right\|_{T V}=1$.

Given the above observation, zero probability is allocated to $H_{0 h}$ by the total variation 
component and we can rewrite our null hypothesis as follows:

$$
\begin{aligned}
H_{0 h}:\left\|G_{h}-G_{h-1}\right\|_{T V} & =\pi_{h}\left\|G_{h}^{*}-G_{h-1}\right\|_{T V} \\
& =\pi_{h} \leq \epsilon .
\end{aligned}
$$

We can then control the prior probability allocated to $H_{0 h}$ by assigning a prior to $\pi_{h}$ which is a mixture of its distribution under $H_{0 h}$ and $H_{1 h}: \pi_{h}>\epsilon$. Let $\zeta_{h}$ be a latent indicator variable which equals 1 if the local null hypothesis $H_{0 h}$ is true and 0 otherwise and let $p_{0 h}=P\left(\zeta_{h}=1\right)$. Our prior on $\pi_{h}$ conditional on $\zeta_{h}$ is then expressed as the following mixture, often referred to as a spike-and-slab prior:

$$
\pi_{h} \mid \zeta_{h} \sim \zeta_{h} \cdot \operatorname{Unif}(0, \epsilon)+\left(1-\zeta_{h}\right) \cdot \operatorname{Unif}(\epsilon, 1), h=2, \ldots, d .
$$

Under the above mixture prior on $\pi_{h}, \mathbf{p}_{0}=\left(p_{02}, \ldots, p_{0 d}\right)^{\top}$ is chosen to reflect prior information about the probability of the global null hypothesis $H_{0}: \zeta_{2}, \ldots, \zeta_{d}=1$ being true. For example, if we have no a priori reason to prefer either the global null or alternative, one could specify $\mathbf{p}_{0}$ so that equal prior probability is placed on each hypothesis, i.e. $P\left(H_{0}\right)=$ $\prod_{h=2}^{d} p_{0 h}=0.5$. As an additional benefit, setting the prior so that the global null and alternative hypotheses have equal probability controls the familywise error rate (FWE) under multiple comparisons (Westfall, Johnson, \& Utts, 1997). One potential method for doing so would be the Bayesian Bonferroni method of Westfall et al. (1997) which assumes $p_{02}=\ldots=p_{0 d}=0.5^{1 /(d-1)}$. Bonferroni type methods, including the preceding prior, have the undesirable feature of being overly conservative when a large number of tests are performed. By this we mean that for large $d$ the prior probability assigned to each local null $H_{0 h}$ is nearly 1 , implying that very strong evidence in required to reject any local null hypothesis.

An alternative to the Bayesian Bonferroni method would be the model space prior proposed by Hans and Dunson (2005) and utilized by Pennell and Dunson (2008). This prior induces dependency between the local null hypotheses by assuming a priori that the $p_{0 h}$ are all equal, i.e. $p_{0 h}=p_{0}$ with $p_{0} \sim \operatorname{Be}\left(a_{p}, b_{p}\right)$. The dependency induced by this 
prior can be understood by noting that the probability of a local null hypothesis being true conditional on the truth value of other local nulls is given by $P\left(\zeta_{h}=1 \mid\left\{\zeta_{j}: j \neq h\right\}\right)=$ $\left(a_{p}+\sum_{j \neq h} \zeta_{j}\right) /\left(a_{p}+b_{p}+d-2\right)$ (Pennell \& Dunson, 2008). In layman's terms, when several local null hypotheses $H_{0 j}, j \neq h$ are true then the local null $H_{0 h}$ is more likely to be true. This feature is conceptually appealing in the analysis of ordinal treatment data because the absence of an effect of treatment for levels less than $h$ would suggest that there is likely no effect of treatment level $h$ as well.

As stated above, it is generally advised that the prior be set so that the global null and alternative have equal probability, i.e. $P\left(H_{0}\right)=0.5$. As such, we wish to specify values for $a_{p}$ and $b_{p}$ in the model state prior so that, on average, the equality $P\left(H_{0}\right)=0.5$ holds. This implies that $a_{p}$ and $b_{p}$ must satisfy:

$$
\begin{aligned}
E\left[P\left(H_{0}\right)\right] & =E\left[P\left(\zeta_{2}=\cdots=\zeta_{d}=1\right)\right] \\
& =E\left[p_{0}^{d-1}\right] \\
& =\int_{0}^{1} p_{0}^{d-1} \frac{\Gamma\left(a_{p}+b_{p}\right)}{\Gamma\left(a_{p}\right) \Gamma\left(b_{p}\right)} p_{0}^{a_{p}-1}\left(1-p_{0}\right)^{b_{p}-1} d p_{0} \\
& =\frac{\Gamma\left(a_{p}+b_{p}\right) \Gamma\left(a_{p}+d-1\right)}{\Gamma\left(a_{p}\right) \Gamma\left(a_{p}+b_{p}+d-1\right)} .
\end{aligned}
$$

We solve the equation $E\left[P\left(H_{0}\right)\right]=0.5$ under the constraint that $a_{p}+b_{p}=1$ which represents unit information in the prior.

\subsubsection{Hypothesis Testing Under the DMDP Model}

The previous two sections have been concerned, respectively, with explicit statement of relevant DMDP hypotheses and appropriate prior specification. Prior specification was accomplished in terms of the probability that local null hypotheses $H_{0 h}: G_{h}=G_{h-1}$, represented by indicators $\zeta_{h}$, are true. We can reinterpret local hypothesis tests in terms of the posterior probability that these indicator variables equal 1, i.e.

$$
\hat{P}\left(H_{0 h} \mid \text { Data }\right)=\hat{P}\left(\zeta_{h}=1 \mid \text { Data }\right)
$$


Because model fitting for the DMDP model is accomplished via MCMC, Rao-Blackwellized estimates (Gelfand \& Smith, 1990) of the posterior probability of null hypotheses being true are readily available. Assuming we have $Q$ MCMC samples from the posterior distribution, the posterior probability for this null hypothesis can be calculated as

$$
\begin{aligned}
\hat{P}\left(H_{0 h} \mid \text { Data }\right) & =\hat{P}\left(\zeta_{h}=1 \mid \text { Data }\right) \\
& =\frac{1}{Q} \sum_{q=1}^{Q} \widetilde{P}^{(q)}\left(\zeta_{h}=1 \mid p_{0}^{(q)}, \mathbf{C}^{(q)}\right),
\end{aligned}
$$

where the superscript $(q)$ denotes the $q^{\text {th }}$ MCMC sample of a value and $\mathbf{C}$ indicates which mixture group each subject belongs to. The form for $\widetilde{P}^{(q)}\left(\zeta_{h}=1 \mid p_{0}^{(q)}, \mathbf{C}^{(q)}\right)$ is given in the discussion of the MCMC sampler in the following section (see 5.2.11).

We can also calculate posterior probabilities for intersections of the local null hypotheses. As an example, consider the null hypothesis of no difference between treatment levels $c$ and $h$ with $c<h$, i.e. $H_{0}: \forall j=c+1, \ldots, h, \pi_{j} \leq \epsilon$. Both the global null and treatment-vscontrol null hypotheses are special cases of this example. The Rao-Blackwellized estimate of the posterior probability of such null hypotheses are given by:

$$
\begin{aligned}
\hat{P}\left(H_{0} \mid \text { Data }\right) & =\hat{P}\left(\zeta_{c+1}=, \cdots,=\zeta_{h}=1 \mid p_{0}^{(q)}, \mathbf{C}^{(q)}\right) \\
& =\frac{1}{Q} \sum_{q=1}^{Q} \widetilde{P}^{(q)}\left(\zeta_{c+1}=, \cdots,=\zeta_{h}=1 \mid p_{0}^{(q)}, \mathbf{C}^{(q)}\right) \\
& =\frac{1}{Q} \sum_{q=1}^{Q} \prod_{j=c+1}^{h} \widetilde{P}^{(q)}\left(\zeta_{j}=1 \mid p_{0}^{(q)}, \mathbf{C}^{(q)}\right) .
\end{aligned}
$$

The final equality above holds because the conditional posterior distributions for the $\zeta_{h}$ are in fact independent.

A final object of interest is the Lowest Observed Effect Level (LOEL) which is the lowest treatment level for which there is evidence of a difference compared to control, i.e. the LOEL is the smallest treatment level $h$ s.t. $\pi_{h}>\epsilon$. The probability that the LOEL 
equals a given treatment level $h$ can be calculated as

$$
\begin{aligned}
\hat{P}(\text { LOEL }=h \mid \text { Data }) & =\frac{1}{Q} \sum_{q=1}^{Q} \widetilde{P}^{(q)}\left(\zeta_{2}=, \cdots,=\zeta_{h-1}=1, \zeta_{h}=0 \mid p_{0}^{(q)}, \mathbf{C}^{(q)}\right) \\
& =\frac{1}{Q} \sum_{q=1}^{Q}\left[\widetilde{P}^{(q)}\left(\zeta_{j}=0 \mid p_{0}^{(q)}, \mathbf{C}^{(q)}\right) \prod_{j=2}^{h-1} \widetilde{P}^{(q)}\left(\zeta_{j}=1 \mid p_{0}^{(q)}, \mathbf{C}^{(q)}\right)\right] .
\end{aligned}
$$

It is also possible that none of the specified treatment levels have a statistically significant difference from control and we would be interested in the probability that the LOEL is greater than $d$. This value can be calculated similarly as

$$
\begin{aligned}
\hat{P}(\text { LOEL }>d \mid \text { Data }) & =\frac{1}{Q} \sum_{q=1}^{Q} \widetilde{P}^{(q)}\left(\zeta_{2}=, \cdots,=\zeta_{d}=1 \mid p_{0}^{(q)}, \mathbf{C}^{(q)}\right) \\
& =\frac{1}{Q} \sum_{q=1}^{Q}\left[\prod_{j=2}^{d} \widetilde{P}^{(q)}\left(\zeta_{j}=1 \mid p_{0}^{(q)}, \mathbf{C}^{(q)}\right)\right] .
\end{aligned}
$$

As stated in Section 5.1.2, the model space prior inherently adjusts posterior estimates

of the probability of various hypotheses for multiple comparisons. As such, the values from the preceding methods can be used for inference directly. It is common to conclude that there is significant evidence against a given null hypothesis if its posterior probability is less than some given cutoff, e.g. $p<0.05$. One of the benefits of the Bayesian methodology, however, is the valid interpretation of p-values as probabilities of various hypotheses being true. As such we recommend that the posterior probability of hypotheses always be included in an analysis due to the fact that even moderate p-values $0.05 \leq p<0.1$ are at least weak evidence against a null hypotheses.

\subsection{Dynamic Mixture of Dirichlet Processes for Threshold Regression}

To this point in the current chapter our discussion of the DMDP model has not assumed a data generating distribution $F$. We now wish to develop a DMDP model which relates an ordinal treatment to event times but can also account for potential confounding in treatment 
assignment. Consider, as a motivating example, a hypothetical observational study looking at the relationship between time to progression of non small cell (NSC) lung cancer and different levels of a chemotherapy drug. It is possible in such a setting that the level of treatment is related to disease progression at treatment or diagnosis. It might be the case that patients with more advanced disease are more likely to be prescribed a higher dose of therapy. We wish to develop a model capable of accounting for such baseline confounding in survival studies with ordinal treatments.

In order to accomplish the above stated goal, we will apply the DMDP methodology to the TR model (2.4.1). We will assume that time-to-event data arise from the $I G$ density conditional on subject specific parameters $\left(x_{0 h i}, \mu_{h i}\right)$. We assume that treatment level is associated with long term trends in subject health $\left(\mu_{h i}\right)$ and that any potential confounding is associated with baseline health $\left(x_{0 h i}\right)$ at time of treatment. We assume that all potential confounders are captured by subject-specific covariates $\mathbf{v}_{h i}$. While a parametric TR model with the described structure is fairly straightforward to specify, such a model will often fail to adequately capture more general shape changes across treatment levels. We can apply the DMDP methodology to produce a model capable of capturing a wide variety of location shift and shape change effects in survival. Stated symbolically, we are interested in the following model:

$$
\begin{aligned}
& T_{h i} \mid x_{0 h i}, \mu_{h i} \stackrel{i n d}{\sim} I G\left(x_{0 h i}, \mu_{h i}\right), \\
& \log \left(x_{0 h i}\right)=\mathbf{v}_{h i}^{\top} \boldsymbol{\alpha}, \\
& \boldsymbol{\alpha} \sim N\left(\boldsymbol{\mu}_{\alpha}, \boldsymbol{\Sigma}_{\alpha}\right), \\
& \mu_{h i} \stackrel{i n d}{\sim} G_{h}, \\
& G_{h}=\left(1-\pi_{h}\right) G_{h-1}+\pi_{h} G_{h}^{*}, \\
& G_{h}^{*} \sim \operatorname{DP}\left(\gamma_{h} G_{0}\right) .
\end{aligned}
$$

We refer to (5.2.1) as the Dynamic Mixture of Dirichlet Processes for Threshold Regression (DMDP-TR) model. Analogous to our previous applications of the DP, we can improve flexibility by modeling the innovation distributions $G_{h}^{*}$ hierarchically. As such, we complete 
the DMDP-TR model by specifying the following priors:

$$
\begin{aligned}
& \gamma_{h} \stackrel{i i d}{\sim} \mathrm{Ga}\left(a_{\gamma}, b_{\gamma}\right), \\
& G_{0} \stackrel{D}{=} N\left(\mu, \sigma^{2}\right), \\
& \mu \sim N\left(\mu_{0}, \sigma_{0}^{2}\right), \\
& \sigma^{2} \sim \operatorname{Inv}-\mathrm{Ga}\left(a_{\sigma}, b_{\sigma}\right) .
\end{aligned}
$$

We will generally want to specify diffuse priors on $\boldsymbol{\alpha}, \mu$, and $\sigma^{2}$. We will also tend to favor priors which put more weight on smaller values $\gamma_{h}$. This will result in a model with relatively fewer mixing components which will generally be sufficient to capture even complicated densities. As an example, we utilize the following prior formulation in our later simulation study and data example:

$$
\begin{aligned}
\left(\boldsymbol{\mu}_{\alpha}, \boldsymbol{\Sigma}_{\alpha}\right) & =(\mathbf{0}, 10000 \cdot \mathbf{I}) \\
\left(a_{\gamma}, b_{\gamma}\right) & =(1,1) \\
\left(\mu_{0}, \sigma_{0}^{2}\right) & =(0,10000) \\
\left(a_{\sigma}, b_{\sigma}\right) & =\left(10^{-5}, 10^{-5}\right)
\end{aligned}
$$

\subsubsection{MCMC Sampler for the DMDP-TR model}

The preceding section was dedicated to introducing the DMDP-TR model. We are currently interested in developing an efficient MCMC sampler which can be used for model fitting. As was the case with the DPM-TR model, this endeavor will be greatly facilitated by restating the model in terms of the Truncation Approximation for the DP. In order to accomplish such a restatement, we will need to think carefully about potential clustering of the drift paremeters $\mu_{h i}$ which can occur on two levels. First, $\mu_{h i}$ belongs to one of the innovation

distributions $G_{j}^{*}$ with $j \leq h$. Secondly, because we assume that $G_{j}^{*}$ is a draw from the Dirichlet Process, $\mu_{h i}$ must be equal to one of the atoms $\mu_{j k}^{*}$ which define $G_{j}^{*}$. To capture this clustering behavior we utilize a cluster indicator vector $\mathbf{C}_{h i}=\left(C_{h i 1}, C_{h i 2}\right)$ which equals $(j, k)$ if $\mu_{h i}$ belongs to atom $k$ in innovation distribution $G_{j}^{*}$. The DMDP-TR model can 
then be restated using cluster notation as follows:

$$
\begin{aligned}
& T_{h i} \mid x_{0 h i}, \mu_{h i} \stackrel{i n d}{\sim} I G\left(x_{0 h i}, \mu_{h i}\right), \\
& \log \left(x_{0 h i}\right)=\mathbf{v}_{h i}^{\top} \boldsymbol{\alpha}, \\
& \boldsymbol{\alpha} \sim N\left(\boldsymbol{\mu}_{\alpha}, \boldsymbol{\Sigma}_{\alpha}\right), \\
& \mu_{h i} \mid\left(\mathbf{C}_{h i}=(j, k)\right)=\mu_{j k}^{*}, \\
& \mu_{j k}^{*} \sim G_{0}, \\
& P\left(\mathbf{C}_{h i}=(j, k)\right)=w_{h j} p_{j k}, \\
& w_{h j}=\pi_{j} \prod_{m=j+1}^{h}\left(1-\pi_{m}\right) \text {, } \\
& \mathbf{p}_{h}=\left(p_{h 1}, \ldots, p_{h K}\right) \sim \pi\left(\mathbf{p}_{h} \mid \gamma_{h}\right), \\
& \gamma_{h} \sim \operatorname{Gamma}\left(a_{\gamma}, b_{\gamma}\right), \\
& G_{0} \stackrel{D}{=} N\left(\mu, \sigma^{2}\right), \\
& \mu \sim N\left(\mu_{0}, \sigma_{0}^{2}\right) \\
& \sigma^{2} \sim \operatorname{Inv-Gamma}\left(a_{\sigma}, b_{\sigma}\right), \\
& \pi_{h} \mid \zeta_{h} \sim \zeta_{h} \cdot \operatorname{Unif}(0, \epsilon)+\left(1-\zeta_{h}\right) \cdot \operatorname{Unif}(\epsilon, 1), \\
& \zeta_{h} \sim \operatorname{Bern}\left(p_{0}\right) \\
& p_{0} \sim \operatorname{Be}\left(a_{p}, b_{p}\right) .
\end{aligned}
$$

It is worth stating explicitly that $\pi\left(\mathbf{p}_{h} \mid \gamma_{h}\right)$ is the same as in previous uses of the Truncated Approximation (see Section 3.3.4).

Given the above representation of the DMDP-TR model, the following sampling scheme follows fairly easily. This ease arises from the fact that many of the sampling steps are directly analogous to sampling steps for the DPM-TR model. When this is the case we explicitly note it. As in previous chapters, we assume that event times $t_{h i}$ and event indicators $d_{h i}$ are observed.

1. Sample all cluster-specific drift terms $\left\{\mu_{11}^{*}, \ldots, \mu_{d K}^{*}\right\}$ conditional on cluster membership $\mathbf{C}=\left\{C_{11}, \ldots, C_{d K}\right\}$. Recalling that subjects are assumed independent conditional on 
their subject specific parameter values, the following expression easily follows:

$$
\pi\left(\mu_{j k}^{*} \mid \mathbf{C}, \boldsymbol{\alpha}, \mathbf{t}, \mathbf{d}\right) \propto d G_{0}\left(\mu_{j k}^{*}\right) \prod_{\left\{(h, i): \mathbf{C}_{h i}=(j, k)\right\}} L\left(\boldsymbol{\alpha}, \mu_{j k}^{*} \mid t_{h i}, d_{h i}\right)
$$

where $\mu_{j k}^{*}$ represents the drift parameter for cluster $j k$. Computation of the likelihood $L$ is equivalent to that for the TR model (2.3.9) except limited to data currently assigned to cluster $j k$, i.e.

$$
\begin{aligned}
L\left(\boldsymbol{\alpha}, \mu_{j k}^{*} \mid t_{h i}, d_{h i}\right) & =\left[f\left(t_{h i} \mid \exp \left\{\mathbf{v}_{h i}^{\top} \boldsymbol{\alpha}\right\}, \mu_{j k}^{*}\right)\right]^{d_{h i}} \\
& \times\left[S\left(t_{h i} \mid \exp \left\{\mathbf{v}_{h i}^{\top} \boldsymbol{\alpha}\right\}, \mu_{j k}^{*}\right)\right]^{1-d_{h i}} .
\end{aligned}
$$

Note that in the case that cluster $j k$ is empty $\left(\mu_{j k}^{*} \mid \mathbf{C}, \boldsymbol{\alpha}\right) \sim G_{0}$. We can use the Random Walk Metropolis (RWM) algorithm to iteratively sample from (5.2.5) for each cluster $j k$.

2. Sample a new value for $\boldsymbol{\alpha}$. Straightforward application of Bayes' Theorem gives a form similar to (5.2.5):

$$
\left(\boldsymbol{\alpha} \mid \boldsymbol{\mu}^{*}, \mathbf{C}, \mathbf{t}, \mathbf{d}\right) \propto \pi(\boldsymbol{\alpha}) \prod_{h=1}^{d} \prod_{i=1}^{n_{j}} L\left(\boldsymbol{\alpha}, \mu_{\mathbf{C}_{h i}}^{*} \mid t_{h i}, d_{h i}\right) .
$$

Random Walk Metropolis samples can be taken from (5.2.7).

It is important to note that conditional on cluster membership $\mathbf{C}$, Steps 1 and 2 for the DMDP-TR model here are completely analogous to the first two MCMC sampling steps $(4.3 .2,4.3 .6)$ for the DMP-TR model with no covariates associated with drift and no heterogeneity in the initial state regression coefficients. As such, the reader is directed to the MCMC sampling procedure for the DMP-TR model for further comment on these first two steps.

3. Sample new values for cluster membership of each subject from

$$
P\left(\mathbf{C}_{h i}=(j, k) \mid \cdot\right)=p_{j k i}^{*},
$$


where

$$
p_{j k i}^{*} \propto\left(w_{h j} p_{j k}\right) \cdot L\left(\boldsymbol{\alpha}, \mu_{j k}^{*} \mid t_{h i}, d_{h i}\right) .
$$

The justification for the form of (5.2.8) is the same as that for Step 3 in the MCMC sampling scheme for the DPM-TR model (3.3.15).

4. Sample new values for all $\pi_{h}$ according to the following distribution:

$$
\pi\left(\pi_{h} \mid \cdot\right)=\zeta_{h} \cdot \frac{\operatorname{Be}\left(\pi_{h} \mid a_{h}^{*}, b_{h}^{*}\right)}{\Phi_{h}(\epsilon)} 1\left(0 \leq \pi_{h} \leq \epsilon\right)+\left(1-\zeta_{h}\right) \cdot \frac{\operatorname{Be}\left(\pi_{h} \mid a_{h}^{*}, b_{h}^{*}\right)}{1-\Phi_{h}(\epsilon)} 1\left(\epsilon<\pi_{h} \leq 1\right),
$$

where

$$
\begin{aligned}
a_{h}^{*} & =1+\sum_{j=h}^{d} \sum_{i=1}^{n_{j}} 1\left(C_{j i 1}=h\right), \\
b_{h}^{*} & =1+\sum_{j=h}^{d} \sum_{i=1}^{n_{j}} 1\left(C_{j i 1}<h\right), \\
\Phi_{h}(\epsilon) & =\int_{0}^{\epsilon} \operatorname{Be}\left(x \mid a_{h}^{*}, b_{h}^{*}\right) d x .
\end{aligned}
$$

The form of this sampling step is established by a rather straightforward application of Bayes' theorem.

5. Sample new values for all $\zeta_{h}$ from $\left(\zeta_{h} \mid \cdot\right) \sim \operatorname{Bern}\left(p_{0 h}^{*}\right)$, where

$$
p_{0 h}^{*}=\frac{p_{0} \cdot \Phi_{h}(\epsilon)}{p_{0} \cdot \Phi_{h}(\epsilon)+\frac{\epsilon}{1-\epsilon}\left(1-p_{0}\right)\left(1-\Phi_{h}(\epsilon)\right)} .
$$

This form for the conditional posterior for $p_{0 h}^{*}$ is again a direct application of Bayes' theorem.

6. Sample a new value for $p_{0}$ according to

$$
\left(p_{0} \mid \cdot\right) \sim \operatorname{Beta}\left(a_{p}+\sum_{h=2}^{d} \zeta_{h}, b_{p}+d-1-\sum_{h=2}^{d} \zeta_{h}\right) .
$$

7. Sample new values for the random weights $\mathbf{p}_{h}=\left(p_{h 1}, \ldots, p_{h K}\right)$ according to the fol- 
lowing algorithm:

$$
\begin{aligned}
& p_{h k}=V_{h k} \prod_{j=1}^{k-1}\left(1-V_{h j}\right) \text { for } k=1, \ldots, K^{*}-1, \\
& p_{h K^{*}}=1-\sum_{k=1}^{K^{*}-1} p_{h k}, \\
& V_{h k} \stackrel{i n d}{\sim} \operatorname{Beta}\left(a_{h k}, b_{h k}\right), \\
& a_{h k}=1+m_{h k}, \\
& b_{h k}=\gamma_{h}+M_{h k},
\end{aligned}
$$

where $m_{h k}=\left|\left\{i: \mathbf{C}_{i}=(h, k)\right\}\right|$ records the number of subjects assigned to group $h k$ and $M_{h k}=\sum_{j=k+1}^{K} m_{h j}$. This sampling scheme comes from the assumption of the Truncation Approximation measure (see Section 3.3.4) and is equivalent to Step 5 in the DMP-TR sampler.

8. Sample a new value for the precision parameters $\gamma_{h}$. If a Gamma prior is chosen, i.e. $\pi\left(\gamma_{h}\right) \stackrel{D}{=} \mathrm{Ga}\left(a_{\gamma}, b_{\gamma}\right)$, then the posterior conditional distribution for $\gamma_{h}$ simplifies to $\left(\gamma_{h} \mid \mathbf{p}_{h}=\left(p_{h 1}, \ldots, p_{h K}\right)\right) \sim \operatorname{Ga}\left(a_{\gamma}+K-1, b_{\gamma}-\log p_{h K}\right)$. Because of the potential for numerical issues when calculating $\log p_{h K}$, we sample from the equivalent distribution

$$
(\gamma \mid \mathbf{p}) \sim G a\left(a+K-1, b-\sum_{k=1}^{K-1} \log \left(1-V_{h k}\right)\right),
$$

which has the simple additional requirement that the $V_{k}$ values be temporarily stored. This step is equivalent to Step 6 in the DPM-TR sampler.

9. Sample new values for the hyper-parameters controlling the baseline prior $G_{0}$. This step is a direct application of Bayes' theorem because we have a sample $\boldsymbol{\mu}^{*}=\left\{\mu_{h k}^{*}\right\}$ from $G_{0} \stackrel{d}{=} N\left(\mu, \sigma^{2}\right)$. The standard Gibbs sample for the conjugate normal case is sufficient:

$$
\begin{gathered}
(\mu \mid \cdot) \sim N\left(\mu_{p}, \sigma_{p}^{2}\right), \\
\mu_{p}=\left(1 / \sigma_{0}^{2}+n / \sigma^{2}\right)^{-1}\left(\mu_{0} / \sigma_{0}^{2}+n \bar{\mu}^{*} / \sigma^{2}\right), \\
\sigma_{p}^{2}=\left(1 / \sigma_{0}^{2}+n / \sigma^{2}\right)^{-1}
\end{gathered}
$$


where $\bar{\mu}^{*}$ is the sample mean of $\boldsymbol{\mu}^{*}$. Our prior for $\sigma_{j}^{2}$ is also conjugate and we again employ the standard Gibbs update:

$$
\begin{gathered}
\left(\sigma^{2} \mid \cdot\right) \sim \operatorname{Inv-Gamma}\left(a_{p}, b_{p}\right), \\
a_{p}=a_{0}+(d K) / 2, \\
b_{p}=b_{0}+\frac{\sum_{h, k}\left(\mu_{h k}^{*}-\mu\right)^{2}}{2} .
\end{gathered}
$$

Repeating steps (1)-(9) for a large number of iterations $Q$ results in a Metropolis within Gibbs sample from the full posterior distribution for the DMDP-TR model.

\subsection{Simulation Study}

We have reiterated throughout this chapter that our main goal has been the development of a time-to-event model capable of detecting both location and shape changes in survival distributions resulting from an ordinal treatment. Additionally, we have argued that the proposed DMDP-TR model has the ability to adjust for potential baseline confounders in non- or partially-randomized studies. In order to validate performance of the proposed model, a simulation study was designed to compare the performance of the DMDP-TR model to standard methods for analyzing such data under a variety of data-generating scenarios. What follows is a review of the simulation study design, including data-generation and model fitting, as well as the most illuminating results.

\subsubsection{Simulated Data}

We begin discussion of the design for our simulation by considering one particular setting where the proposed model will be particularly useful: randomized trials with time-to-event outcomes and ordinal treatment levels. Examples of this type of setting include toxicology studies, such as the NTP urethane study discussed earlier, or dose-finding clinical trials in the development of novel pharmaceuticals. For the purposes of our simulation we will imagine a setting where subjects are randomly assigned to 1 of 4 ordered treatment levels and that a survival time (or censoring time) for each subject is recorded. In this setting 
there are 3 general data cases which will be of interest:

1. The null case where there is no treatment effect.

2. Treatment results in a location shift in the time-to-event distribution.

3. Treatment results in a shape change in the time-to-event distribution.

Further, we might imagine that in Cases 2 and 3 changes between treatment levels might demonstrate a variety of behaviors. Within both cases we will consider subcases (2a and 3a) where each increase in treatment level results in changes in the survival distribution and subcases ( $2 \mathrm{~b}$ and $3 \mathrm{~b}$ ) where only the highest treatment level has an effect compared to the control group.

In addition to the randomized study scenario discussed above, the DMDP-TR model was also developed so as to be applicable in non-randomized settings where survival outcomes are related to an ordinal treatment which might potentially be confounded with covariates associated with baseline health. We might imagine an observational study of the effect of some therapy on survival where subjects with more advanced disease (i.e. those that are less healthy) at baseline are more likely to be prescribed more intense levels of treatment. To accurately estimate the effect of treatment we must control for this confounding. In addition to the three data cases highlighted above, we will further be interested in performance of the DMDP-TR model in two general data cases which exhibit baseline confounding, for a total of 5 data cases:

4. Treatment effect with confounding toward the null hypothesis; i.e. confounding which, if not accounted for, decreases the distance between treatment specific survival curves.

5. No treatment effect with confounding away from the null hypothesis, i.e. confounding which, if not accounted for, increases the distance between treatment specific survival curves.

We will examine two cases of confounding toward the null hypothesis: one case, 4(a), related to location shift effects of treatment and another case, 4(b), related to shape change effects of treatment. 
The remainder of this section will be dedicated to discussing the data generating models used for each of these five general data cases in detail. For each data case we will assume the data consist of observations on 200 subjects, evenly distributed between 4 ordinal treatment levels $h=1, \ldots, 4$. For each subject we observe an event time $t_{h i}$, an event indicator $d_{h i}$, and a potential baseline covariate $v_{h i}$. All data were generated from special cases of the First Hitting Time model due to our desire to consider cases with potential baseline confounding with subject health.

\section{Case 1: No Treatment Effect}

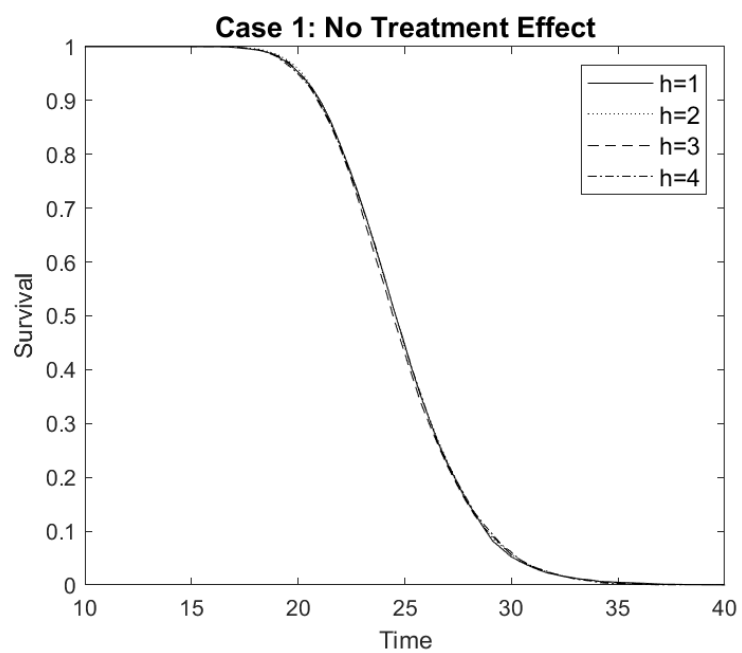

Figure 5.1: True treatment-level survival functions for Case 1 where there is no treatment effect and no confounding.

The first case considered in our simulation study is the null case of no treatment or confounder effects. The need to test the proposed methodology in such a setting is to ensure that Type 1 error is controlled. Data are simulated from the following data-generating 
model:

$$
\begin{gathered}
T_{h i} \sim I G\left(x_{0 h i}, \mu_{h i}\right), \\
x_{0 h i}=\exp (3.9), \\
\mu_{h i} \sim N(-2.0,0.125) .
\end{gathered}
$$

A plot of the true survival functions, separated by dose, used to generate the data can be found in Figure 5.1.

\section{Case 2: Location Shift Effect}
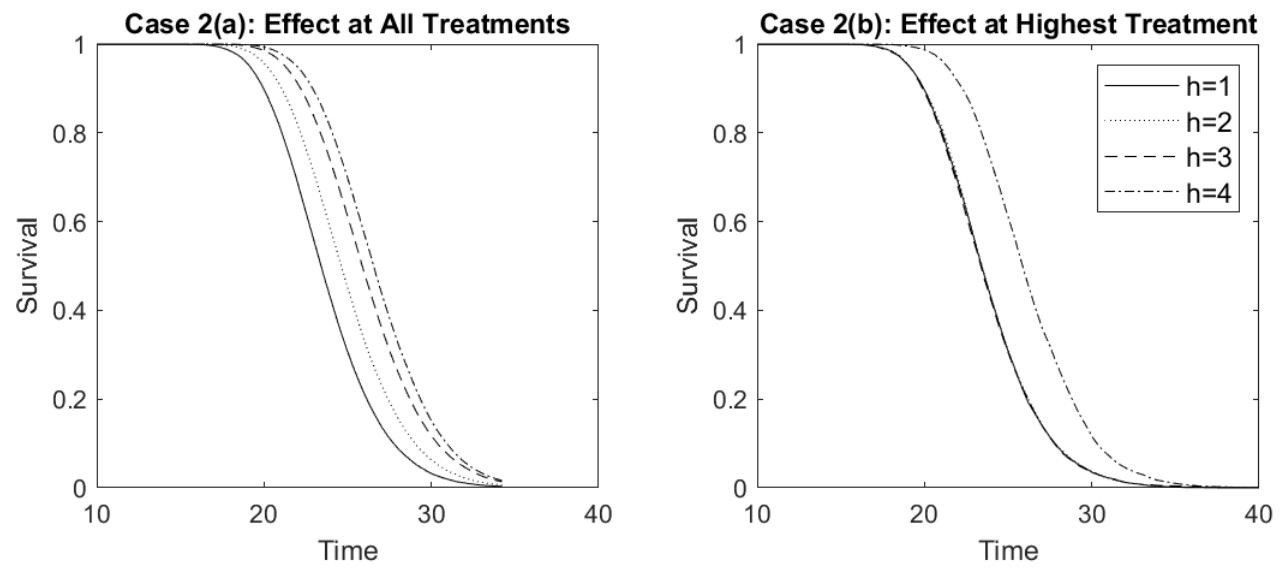

Figure 5.2: True treatment-level survival functions for Case 2 where there is a location shift effect of treatment and no confounding.

Our second general data case involves simple location shifts in survival with changes in treatment level. Data that display such stochastic ordering are ideal in analysis since they generally allow for relatively straightforward conclusions and a wide variety of survival models. As such, any widely applicable survival method should be able to perform well in detecting location shifts in the time-to-event distribution. Simulated data for the location shift cases 2(a) and 2(b) with no confounding were generated from the following models, survival plots for which can be found in Figure 5.2: 
Case 2(a): Location shift effect at all treatment levels:

$$
\begin{gathered}
T_{h i} \sim I G\left(x_{0 h i}, \mu_{h i}\right), \\
x_{0 h i}=\exp (3.9), \\
\mu_{h i} \sim N\left(e_{h}, \sigma_{h}^{2}\right), \\
e_{h}=-2.1 \cdot I\{h=1\}-2.0 \cdot I\{h=2\}-1.9 \cdot I\{h=3\}-1.85 \cdot I\{h=4\}, \\
\sigma_{h}=0.1+0.025(4-h) .
\end{gathered}
$$

Case 2(b): Location shift effect at last treatment level:

$$
\begin{gathered}
T_{h i} \sim I G\left(x_{0 h i}, \mu_{h i}\right), \\
x_{0 h i}=\exp (3.9), \\
\mu_{h i} \sim N\left(e_{h}, \sigma_{h}^{2}\right), \\
e_{h}=-2.1 \cdot I\{h<4\}-1.9 \cdot I\{h=4\}, \\
\sigma_{h}=0.175 \cdot I\{h<4\}+1.25 \cdot I\{h=4\} .
\end{gathered}
$$

It should be noted that the mean and variance of subject specific drifts $\mu_{h i}$ change with treatment level $h$ in the above models. Having both $e_{h}$ and $\sigma_{h}$ vary with treatment was necessary to represent strict location shifts because of the mean-variance relationship in the IG distribution. 


\section{Case 3: Shape Change Effect}
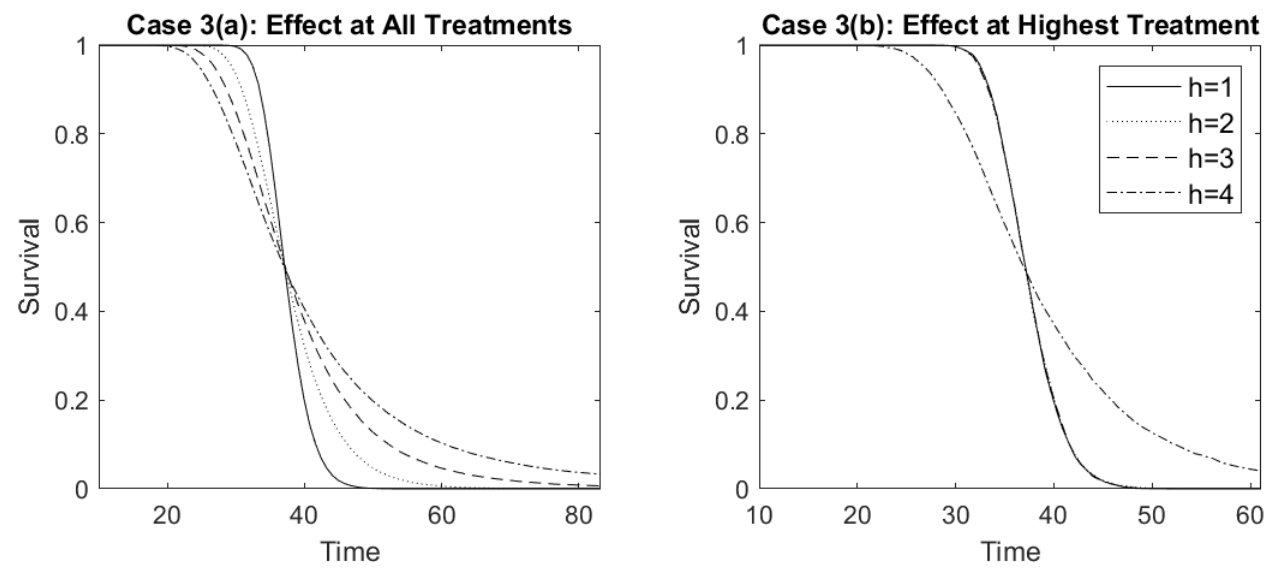

Figure 5.3: True treatment-level survival functions for Case 3 where there is shape change effect of treatment and no confounding.

The ability to capture shape differences in survival curves has long been a focus in the literature due to the tendency of such situations to exhibit "crossing" survival curves. The reason for this prominence in the literature is largely motivated by the fact that crossing survival curves create a variety of problems for analysis when using popular weighted logrank tests. Most notably, crossing survival curves violate the proportionality assumption under which the logrank test has maximum power. The concern of crossing survival curves is particularly prominent in the clinical setting (Bouliotis \& Billingham, 2011) where the DMDP-TR has obvious application. A number of methods for analyzing "crossing" survival data have been proposed, several of which were reviewed by Li et al.(2015).

Given the prominence of crossing survival data cases in the recent clinical survival analysis literature, it is important to examine the performance of the DMDP-TR model in such cases. The goal of this data case was to test performance of the proposed methodology when the shape of the survival curve changes with dose but median survival does not. Such patterns could, for example, be readily produced via changes in tail behavior. A simpler solution, and one which we utilize for our simulation, would involve changes in event time 
variance across dose. We employed the following two data generating models (5.3.4 and 5.3.5) to simulate data for such cases in our study:

Case 3(a): Shape change effect at all treatment levels:

$$
\begin{gathered}
T_{h i} \sim I G\left(x_{0 h i}, \mu_{h i}\right), \\
x_{0 h i}=\exp (5), \\
\mu_{h i} \sim N(-4,0.3 \times h) .
\end{gathered}
$$

Case 3(b): Shape change effect at last treatment level:

$$
\begin{gathered}
T_{h i} \sim I G\left(x_{0 h i}, \mu_{h i}\right), \\
x_{0 h i}=\exp (5), \\
\mu_{h i} \sim N\left(-4, \sigma_{h}^{2}\right), \\
\sigma_{h}=0.3 \cdot I\{h<4\}+0.9 \cdot I\{h=4\} .
\end{gathered}
$$

Plots of the marginal survival by treatment group for both subcases are presented in Figure 5.3.

It should again be noted that the variances of subject specific drifts $\mu_{h i}$ change with treatment level $h$ in the above models. Having $\sigma_{h}$ vary with treatment was necessary to represent scale shifts without accompanying changes in median survival. As was the case in the Case 2(a) and 2(b) models, such model specifications are necessary due to the meanvariance relationship in the IG distribution.

\section{Case 4: Confounding Toward the Null}

The preceding three data cases have all been limited to cases where treatment is the only covariate which affects survival. We now consider the case where a baseline covariate $v_{h i}$ is related to baseline health which is assumed to be confounded with treatment. We are currently interested in situations where this confounding is structured so as to obscure or hide the true positive effect that treatment has on survival; i.e., we are interested in bias towards the null hypothesis. We will simulate data from two different models which have 
such a bias structure. The first model, which we call Case 4(a), is closely related to the location shift model for Case 2(a) in that the model for both cases would be equivalent if the former case did not have a confounding effect for $v_{h i}$. The model for Case 4(a) is set up so that the effect of confounder $v_{h i}$ is such that the marginal treatment-specific survival curves are nearly identical due to differences in the distribution of confounder $v_{h i}$ across treatment groups. In other words, the effect of the confounder completely masks the effect of treatment. This type of behavior can be seen in Figure 5.4. Two plots are included in this figure. The first plot shows the true marginal survival for each treatment group. The second plot shows what those same survival curves would look like if subject specific values of the confounder were $v_{h i}^{*}=0.25$, which is the population mean of the confounder values. Comparisons of these two plots makes obvious the bias induced by confounding which, to reiterate, is towards the null hypothesis.
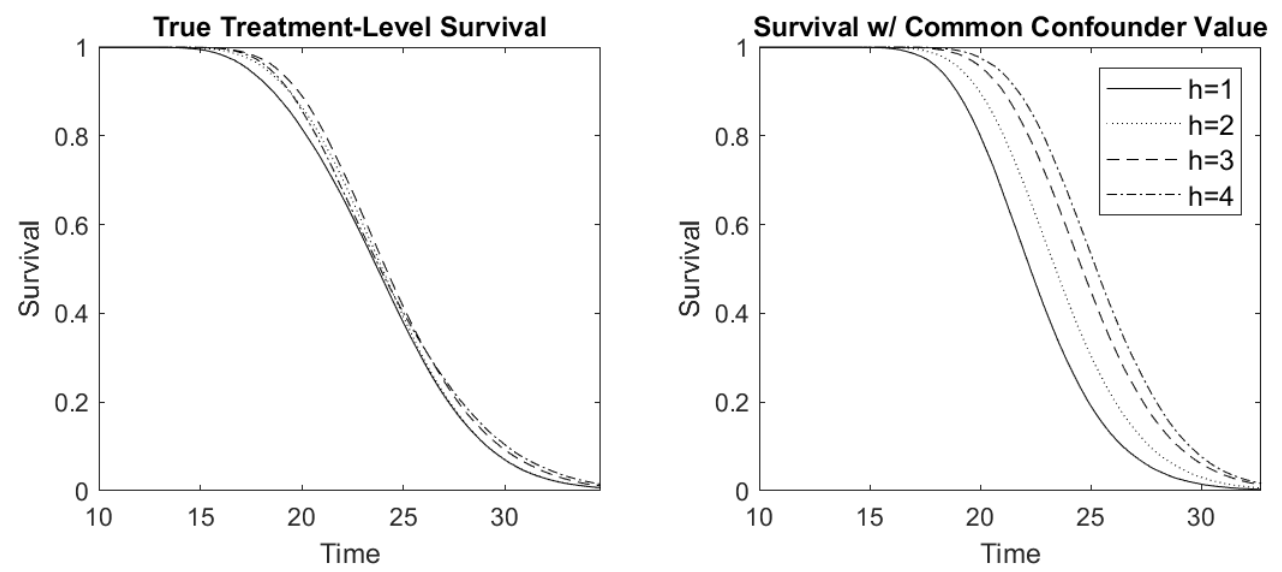

Figure 5.4: True treatment-level survival functions for Case 4(a) are presented in the left plot. The right plot presents what the survival curves would be if all subjects had the same value for the confounder $v_{h i}=0.25$. 
The model which generates the simulated data for Case 4(a) is given by:

$$
\begin{gathered}
T_{h i} \sim I G\left(x_{0 h i}, \mu_{h i}\right), \\
x_{0 h i}=\exp \left(4.1-0.1 \times v_{h i}\right), \\
\mu_{h i} \sim N\left(e_{h}, \sigma_{h}^{2}\right), \\
P\left(v_{h i}=j \mid h\right)=1 / 2 \cdot I\{j=h\}+1 / 6 \cdot I\{j \neq h\}, \\
e_{h}=-2.1 \cdot I\{h=1\}-2.0 \cdot I\{h=2\}-1.9 \cdot I\{h=3\}-1.85 \cdot I\{h=4\}, \\
\sigma_{h}=0.1+0.025(4-h) .
\end{gathered}
$$

It should be noted that the above model (and the models for Cases 4(a) and 5) specify the distribution for the confounder given treatment level; i.e., $[v \mid h]$. This was done for convenience as it made simulating data with equal numbers of subjects in each treatment group easier. While the data were simulated in this manner, the hypothesized data generating mechanism is actually that $v$ is not on the causal pathway between treatment and outcome and is in fact a confounder.

We are also interested in confounding towards the null hypothesis when differences in treatment level result in changes in the shape of the survival function. We must be somewhat careful in specifying a data generating model for this case due to issues related to crossing survival curves. Specifically, it can be quite difficult to specify effects for the confounder that explicitly mask the scale-shift effect of treatment. In fact, this is not a simple task even when treatment merely changes the variance; e.g., Case 3(a). For our current purposes it will suffice to consider a data-generating model as biased towards the null hypothesis if the distances between survival curves for treatments and control would be larger if all subjects shared the same value for the confounder, e.g. $v_{h i}=0.25$. The following model conforms to 
this pattern and is employed in our simulation study to explore the prescribed confounding:

$$
\begin{gathered}
T_{h i} \sim I G\left(x_{0 h i}, \mu_{h i}\right), \\
x_{0 h i}=\exp \left(5.15-0.15 \times v_{h i}\right), \\
\mu_{h i} \sim N\left(e_{h}, \sigma_{h}^{2}\right), \\
P\left(v_{h i}=j \mid h\right)=1 / 2 \cdot I\{j=h\}+1 / 6 \cdot I\{j \neq h\}, \\
e_{h}=-4, \\
\sigma_{h}=0.3 \times h .
\end{gathered}
$$

In order to demonstrate that Model 5.3.7 does in fact include confounding towards the null hypothesis, we can again compare plots of the true treatment specific survival curves to what those survival curves would look like assuming all subjects share the same value for the confounder. Survival plots for both models can be found in Figure 5.5.
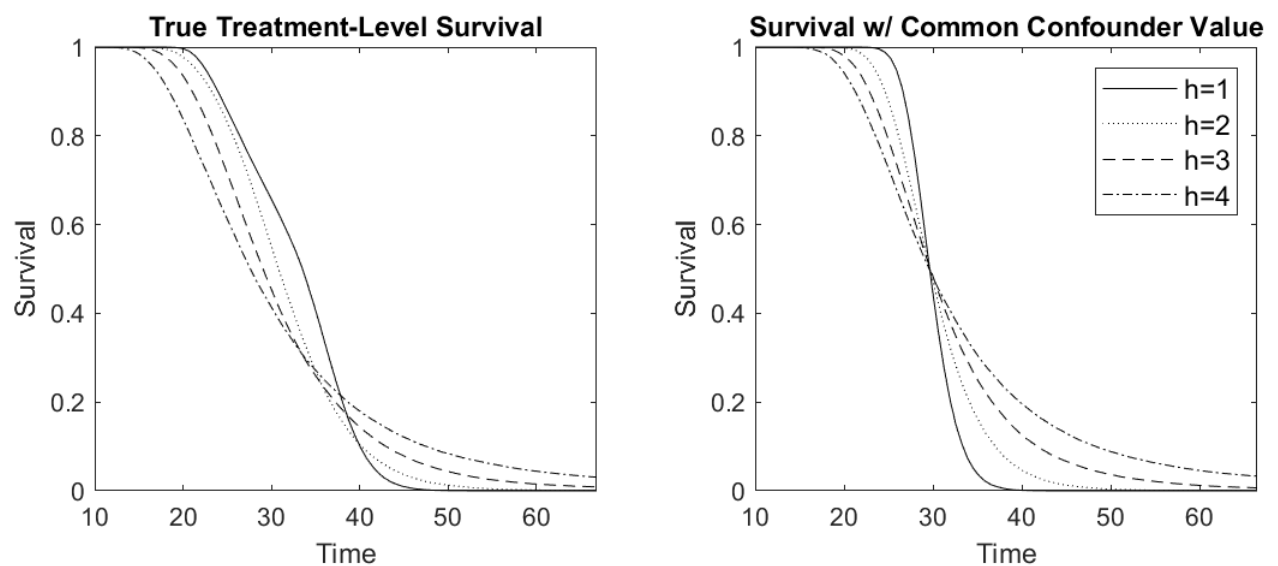

Figure 5.5: True treatment-level survival functions for Case 4(b) are presented in the left plot. The right plot presents what the survival curves would be if all subjects had the same value for the confounder $v_{h i}=0.25$.

In contrast to Case 4(a), the survival plots for Case 4(b) (Figure 5.5) do not make it visually obvious that differences in the confounder between subjects results in treatment level survival curves which are closer to the control survival curve. Thus, we quantify the 
distances between treatment and control survival curves in the two models using the $\mathcal{L}_{2}$ distance. One can see in Table 5.1 that the confounding in Case 4(b) does result in survival curves closer to control across all three treatment groups. As such, we consider this model to have confounding towards the null hypothesis of no treatment effect.

\begin{tabular}{|c|cc|}
\hline Treatment level & Marginal Survival & Survival w/ $v_{h i}=0.25$ \\
\hline$h=2$ & 0.3982 & 0.4160 \\
$h=3$ & 0.6974 & 0.7465 \\
$h=4$ & 0.9918 & 1.0445 \\
\hline
\end{tabular}

Table 5.1: $\mathcal{L}_{2}$ distance between treatment $(h=2,3,4)$ and control for Case $4(\mathrm{~b})$.

\section{Case 5: Confounding Away From the Null}
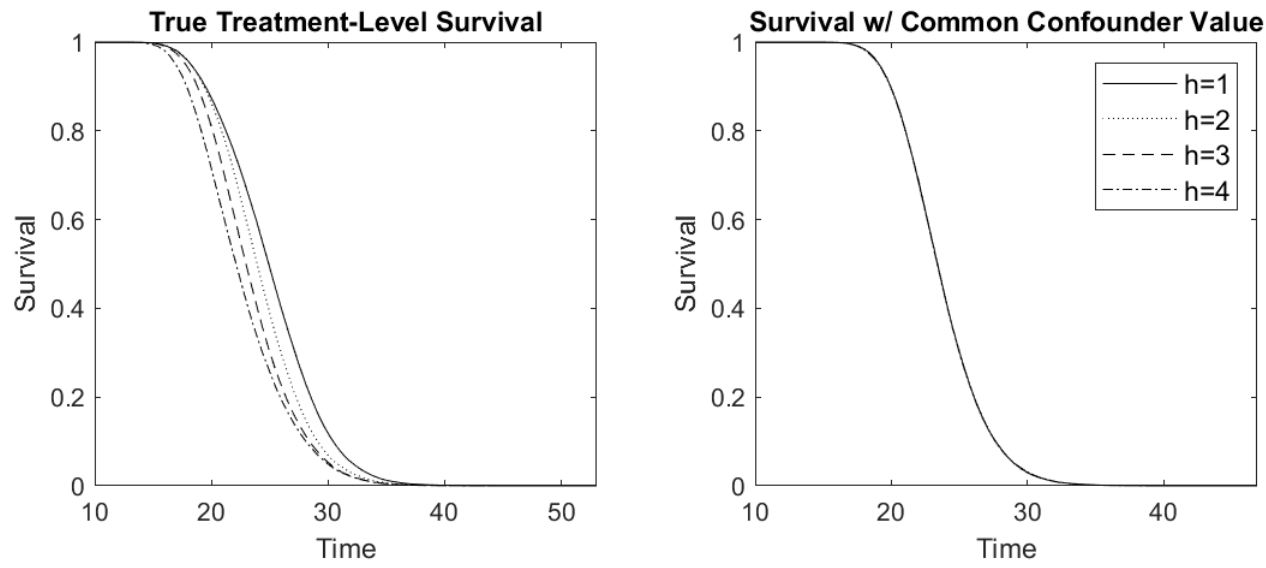

Figure 5.6: True treatment-level survival functions for Case 5 are presented in the left plot. The right plot presents what the survival curves would be if all subjects had the same value for the confounder $v_{h i}=0.25$.

Our final data case attempts to test the proposed methodology when baseline confounding results in an apparent treatment effect where no effect actually exists. If a model is unable 
to appropriately account for such confounding then the Type 1 error rate will be higher than the specified significance level; i.e, the method will be anti-conservative. To simulate data from such a setting we again used a model closely related to that used for Case 2(a):

$$
\begin{gathered}
T_{h i} \sim I G\left(x_{0 h i}, \mu_{h i}\right), \\
x_{0 h i}=\exp \left(4.1-0.1 \times v_{h i}\right), \\
\mu_{h i} \sim N(-2.0,0.125), \\
P\left(v_{h i}=j \mid h\right)=1 / 2 \cdot I\{j=h\}+1 / 6 \cdot I\{j \neq h\} .
\end{gathered}
$$

Model 5.3.8 specifies a location shift effect at all treatment levels with confounding between treatment and initial health. In order to visualize the confounding present in Case 5 we again compare marginal survival plots for the actual data generating model and what those plots would look like if all subjects had the same value for the confounder; e.g., $v_{h i}=0.25$. These plots are included in Figure 5.6 and illustrate that the difference in survival between the treatment groups is complete due to differences in subject-specific values of the confounder.

\section{Censoring and Other Comments}

In the preceding subsections we introduced the 8 specific data cases $(1,2(\mathrm{a}), 2(\mathrm{~b}), 3(\mathrm{a})$, 3(b), 4(a), 4(b), 5) which form the basis of our simulation study. Due to the nature of time-to-event data, we also need to test our methodology under various levels of censoring. We decided to simulate data with no, moderate $(25 \%)$, and high $(50 \%)$ expected censoring. To generate censored data we simulated censoring times from the $I G\left(x_{0}, \mu_{(\cdot)}\right)$ distribution, independent of event times, with initial health parameter equal to $x_{0}=E\left[x_{0 h i}\right]$ and drift values $\mu_{25}$ and $\mu_{50}$ which correspond to moderate and high censoring, respectively. Observation times for the censored data set are then taken to be the minimum of the event and censoring times. Values for $x_{0}, \mu_{25}$ and $\mu_{50}$ for each data case can be found in Table 5.2.

A final comment regarding the number of simulated datasets used in our study is worth making here. For each of the $8 \times 3=24$ combinations of case and censoring level, 100 datasets were simulated for a total of 2,400 datasets. For each dataset a number of models 
were fit. A discussion of which models were explored in our study is the primary focus of the next section.

\begin{tabular}{|l|lll|}
\hline Data Case & $x_{0}$ & $\mu_{25}$ & $\mu_{50}$ \\
\hline 1 & 49.40 & -1.782 & -2.003 \\
$2(\mathrm{a})$ & 49.40 & -1.728 & -1.943 \\
$2(\mathrm{~b})$ & 49.40 & -1.805 & -2.031 \\
$3(\mathrm{a})$ & 148.41 & -3.485 & -4.005 \\
$3(\mathrm{~b})$ & 148.41 & -3.657 & -3.977 \\
$4(\mathrm{a})$ & 47.29 & -1.719 & -1.944 \\
$4(\mathrm{~b})$ & 120.02 & -3.285 & -3.886 \\
5 & 47.26 & -1.750 & -1.980 \\
\hline
\end{tabular}

Table 5.2: Parameter values for the IG models used to create censoring times.

\subsubsection{Analysis Methods}

We next discuss in some depth the methods compared in our simulation study. First we review the two versions of the DMDP-TR model we fit and then discuss in greater depth several weighted logrank based methods included for the sake of comparison.

In our development of the DMDP-TR model (5.2.1), we explicitly included the ability to adjust for potential confounders related to baseline health. We are interested in highlighting the capability of such adjustment. As such, we fit two different DMDP-TR models to each simulated dataset, one which adjusts for potential confounder $v_{h i}$ and one which does not:

1. DMDP-TR unadj follows (5.2.1) with $\mathbf{v}_{h i}^{\top}=1$.

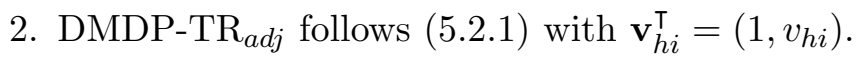

All priors are specified as in example (5.2.2-5.2.3) and a significance level of $\alpha=0.05$ was used for hypothesis testing. 
We also wanted to compare performance of our proposed model with more standard counting process-based methods such as the logrank or Harrington-Fleming tests. The motivation for comparison to this class of methods is twofold. First, counting process-based tests are quite common in survival analysis settings where there are only a limited number of treatment groups, exactly the setting in which the DMDP-TR model is expected to be of value. Secondly, counting process-based methods are non-parametric and, as such, offer a natural benchmark for our semi-parametric methodology. In order for fair comparisons to be made between our model and counting process-based methods, however, the latter should have several similar features. Specifically, we wish to compare our model to a counting process-based method capable of the following:

1. Adjustment for potential confounders.

2. Trend tests, i.e. tests with an ordered alternative hypothesis.

3. P-value adjustment for multiple comparisons.

We can address the first two of the preceding requirements by employing the fairly standard Tarone test (Tarone, 1975) which is essentially the logrank test with an ordered alternative. Adjustment for potential confounders can be accomplished via the standard stratification method of Aalen et al. (2008); Klein and Moeschberger (2003). One unfortunate requirement of such stratification methods is the need for subjects in each treatment groups within each stratum. Such a requirement on the data is not necessary for fitting the DMDP-TR model which associates covariates with the outcome via regression on baseline health. However, in order to make fair comparisons, the simulation data were all generated such that this data requirement was met. With the preceding comments in place, we decided that a thorough comparison of methods would require at least 4 logrank based tests:

1. The logrank test not adjusted for potential confounder $v_{h i}$.

2. The logrank test adjusted for potential confounder $v_{h i}$.

3. The Tarone test for trend not adjusted for potential confounder $v_{h i}$.

4. The Tarone test for trend adjusted for potential confounder $v_{h i}$.

The $\mathbf{R}$ (R Core Team, 2018) package coin (Hothorn, Hornik, van de Wiel, \& Zeileis, 2008) 
was used to perform logrank and Tarone tests, both unadjusted and adjusted for potential confounder $v_{h i}$.

We also compared our DMDP-TR-based stepdown tests to multiple testing procedures based on counting processes. Several many-to-one survival tests have been explored in the literature, at least for the one-way case where there are no potential confounders. Much of this work has focused on applying the general closed testing procedure (CTP) methodology discussed by Marcus, Peritz, and Gabriel (1976). Two examples of such methods are the many-to-one test proposed by Chen (2000) and the all pairwise comparisons method of Logan, Wang, and Zhang (2005). We chose not to compare these methods to our proposed methodology because the former is not designed to test for changes between adjacent treatment groups and the latter is too conservative in that it adjusts for all possible pairwise comparisons.

In an effort to develop an appropriate comparison method, we can think carefully about the nature of trends tests in survival analysis. Consider, for example, the global null and alternative hypotheses for the test of trend in the hazard functions for treatments groups $h=1, \ldots, d:$

$$
H_{0}: \lambda_{1}=\lambda_{2}=\ldots=\lambda_{d} \text { vs. } H_{A}: \lambda_{1} \leq \lambda_{2} \leq \ldots \leq \lambda_{d}
$$

where at least one of the inequalities in the alternative is actually strict. In this setting it is clear that testing for a difference between treatment $j$ and control is equivalent to testing for any trend in treatments less than or equal to $j$, i.e.

$$
H_{0 j}: \lambda_{1}=\lambda_{j} \text { vs. } H_{A j}: \lambda_{1}<\lambda_{j} \Leftrightarrow H_{0 j}: \lambda_{1}=\ldots=\lambda_{j} \text { vs. } H_{A j}: \lambda_{1} \leq \ldots \leq \lambda_{j}
$$

As such, many-to-one trend tests for ordinal treatment data are inherently closed under intersection in the sense that $H_{02} \subset H_{03} \subset \ldots \subset H_{0 d}=H_{0}$. As a consequence, a natural CTP for such settings is the fixed testing sequence (FTS) procedure where individual hypotheses are performed in descending order $\left(H_{0 d}, H_{0(d-1)}, \ldots, H_{0 j^{*}}\right)$ at significance $\alpha$ until 
the first insignificant test $H_{0 j^{*}}$. Under this FTS all treatment groups $j>j^{*}$ are considered significantly different from control at familywise significance $\alpha$. This fixed testing sequence, applied to the Tarone tests of interest, should provide an insightful comparison to our proposed methodology.

The FTS procedure described above will, unfortunately, only work for the trend test setting, i.e. when we perform the Tarone test. Multiple comparisons for the logrank test will employ Holm's procedure (Holm, 1979) since results from Logan et al. (2005) suggest that this method provides performance in the many-to-one setting comparable to closed testing procedures designed for such settings.

In summary, the comparison methods used for our simulation study are the logrank and Tarone tests, with and without adjustment for potential confounder $v_{h i}$. Ordered alternative hypotheses for the Tarone test are of the form $H_{0}: \lambda_{1} \geq \cdots \geq \lambda_{4}$, i.e. increased treatment level is associated with an increase in survival. For the logrank tests we perform multiple testing adjustments using Holm's procedure. The FTS procedure was used to make multiple comparisons adjustment for the Tarone tests. In both cases, testing was performed at a nominal familywise error rate of $\alpha=0.05$.

\subsubsection{Results}

We evaluated our proposed methodology based on both hypothesis testing performance and survival estimation. We begin by reviewing results from the former where we were chiefly interested in behavior of the various methods in rejecting the global null hypothesis of no treatment effect as well as all three treatment-vs-control hypotheses. Figures 5.7-5.8 present the proportion of time that our six methods of interest rejected these hypotheses across data case, effect type, and censoring level. Several patterns are worth noting. First, all three methods which adjust for potential confounder $v_{h i}$ were successful in controlling Type 1 error across all settings (Cases 1 \& 5). In contrast, the unadjusted DMDP and Logrank tests were clearly anti-conservative in the presence of confounding away from the null (Case 5). It should be noted that the unadjusted Tarone test does appear to be controlling Type 1 error in the presence of confounding away from the null. This is a consequence of the fact 
that confounding in this data case resulted in a negative relationship between treatment level and marginal survival in contrast to our specification of the Tarone test with an alternative hypothesis stating a positive relationship. If we had specified a negative relationship in the alternative hypothesis for the Tarone test, then the rejection rate for this test would have been larger than that for the unadjusted Logrank test, i.e. the former would have been more anti-conservative, due to the fact that the former model is a special case of the latter but with a one-sided alternative. As a second point of interest, the DMDP-TR models were considerably more powerful than the Tarone and Logranks tests in detecting scale shift effects (Cases 3(a), 3(b), 4(b)). It is worth noting here that in Cases 3(a) and 3(b) the adjusted DMDP-TR model demonstrated greater power than the unadjusted DMDP-TR model. The DMDP-TR models also provided only marginally less power than the Tarone tests in detecting location shift effects of treatment on survival (Cases 2(a), 2(b), 4(a)). These patterns were somewhat expected in light of earlier comments about the Logrank test, and by extension the Tarone test, being most powerful under proportional hazards. In contrast, the Logrank tests were generally the least powerful method considered, largely due to the fact that these tests have two-sided alternative hypotheses. It is worth noting that both the Logrank and Tarone tests' lack of power in detecting scale shifts is particularly prominent in the presence of high censoring. These observed patterns concerning power hold both in the absence of confounding and when comparing only adjusted models when confounding is present.

Survival estimation was also of interest in assessing the performance of our proposed DMDP-TR methodology. For data cases 1-3 where no confounding occurs, survival estimates were derived for each treatment level $h \in H=\{1, \ldots, 4\}$. For these data cases we summarized survival performance using the following $L_{2}$ error:

$$
L_{2}(\widehat{S})=\left(\sum_{h \in H} \int_{0}^{t_{\max }}(\widehat{S}(t \mid h)-S(t \mid h))^{2} d t\right)^{1 / 2}
$$

where $S$ and $\hat{S}$ are, respectively, the true and estimated survival functions conditional on treatment level $h$. Plots of median $L_{2}$ error for the unadjusted DMDP-TR model and KM 
estimates under data cases 1-3 can be found in Figure 5.9. One can see that the DMDP-TR model generally provides survival estimates that are at least as good as the nonparametric KM method with the lone exception being the scale shift case with no confounding (Case $3(\mathrm{a}))$ and high censoring.

For data cases 4-5, where confounding exists, we estimated survival for each combination of treatment $h \in H=\{1, \ldots, 4\}$ and confounder $v \in V=\{1, \ldots, 4\}$. We then summarized survival error using a similar $L_{2}$ error as before:

$$
L_{2}(\widehat{S})=\left(\sum_{h \in H} \sum_{v \in V} \int_{0}^{t_{\max }}(\widehat{S}(t \mid h, v)-S(t \mid h, v))^{2} d t\right)^{1 / 2}
$$

Plots of median $L_{2}$ error for the adjusted DMDP-TR model and KM estimates under data cases 4-5 can be found in Figure 5.10. We again see that the DMDP-TR model provides improved survival estimation on average compared to KM estimates.

In summary, our simulation study has demonstrated that the proposed DMDP-TR model provides a worthwhile methodology for survival analysis in the context of ordinal treatment data. Under settings where a proportional hazards assumption is reasonable, our proposed model provided power in testing for an ordinal treatment effect which was comparable to standard methods. In settings where a proportional hazards assumption would be inappropriate our proposed method was clearly more powerful than standard methods. Additionally, our proposed model provides survival estimation with average error comparable to, if not better than, nonparametric KM estimates. 

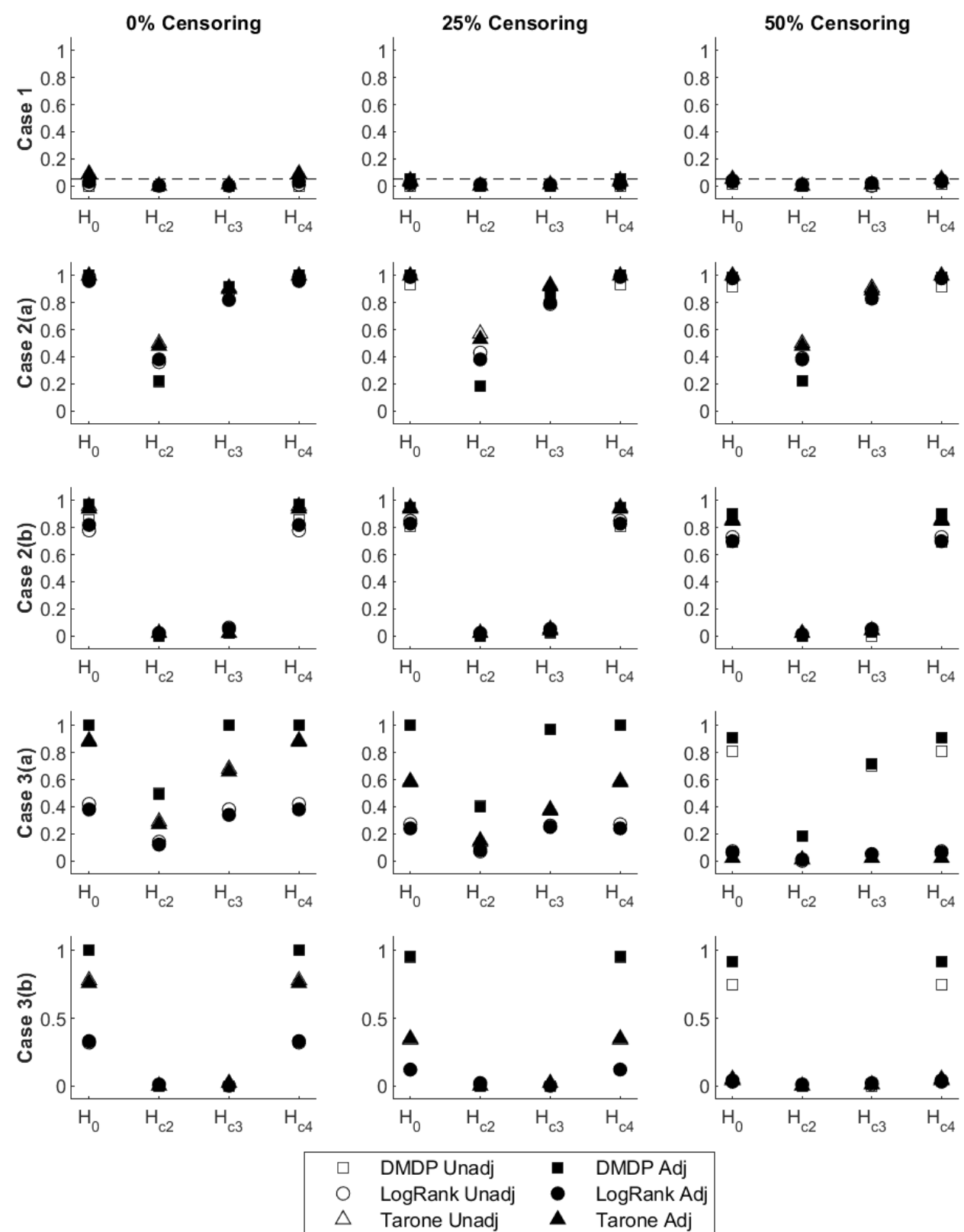

Figure 5.7: Proportion of simulated datasets from non-confounded cases (Cases 1-3) where null hypotheses of interest are rejected, separated by case and censoring level. Results are presented for all 6 fitted models. Unadjusted models are those which did not adjust for potential confounder $v_{h i}$. Adjusted models are those which include confounder adjustment. Dashed lines indicate the value $\alpha=0.05$ which is the significance at which all tests were performed. 

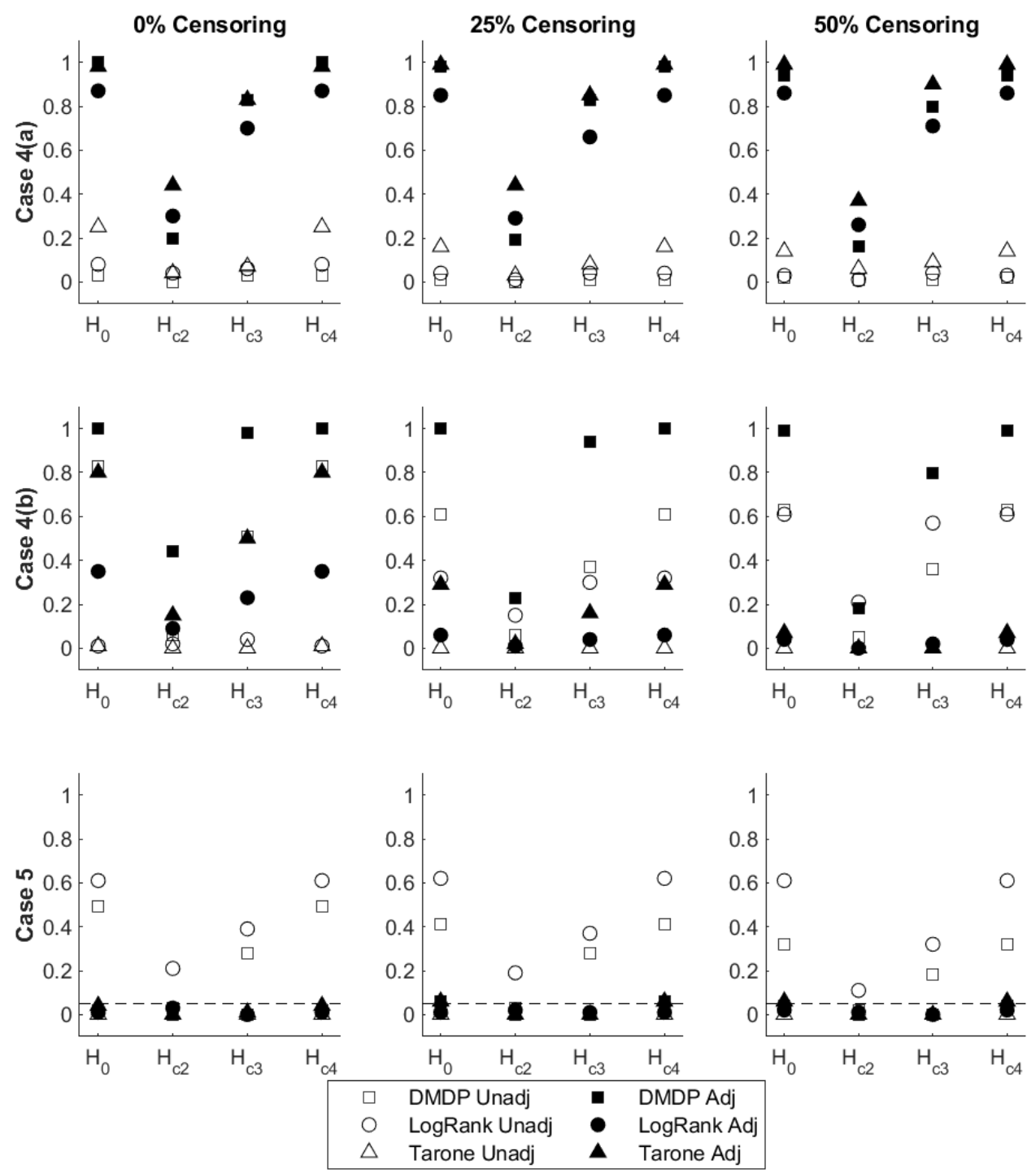

Figure 5.8: Proportion of simulated datasets from confounded cases (Cases 4-5) where null hypotheses of interest are rejected, separated by case and censoring level. Results are presented for all 6 fitted models. Unadjusted models are those which did not adjust for potential confounder $v_{h i}$. Adjusted models are those which include confounder adjustment. Dashed lines indicate the value $\alpha=0.05$ which is the significance at which all tests were performed. 

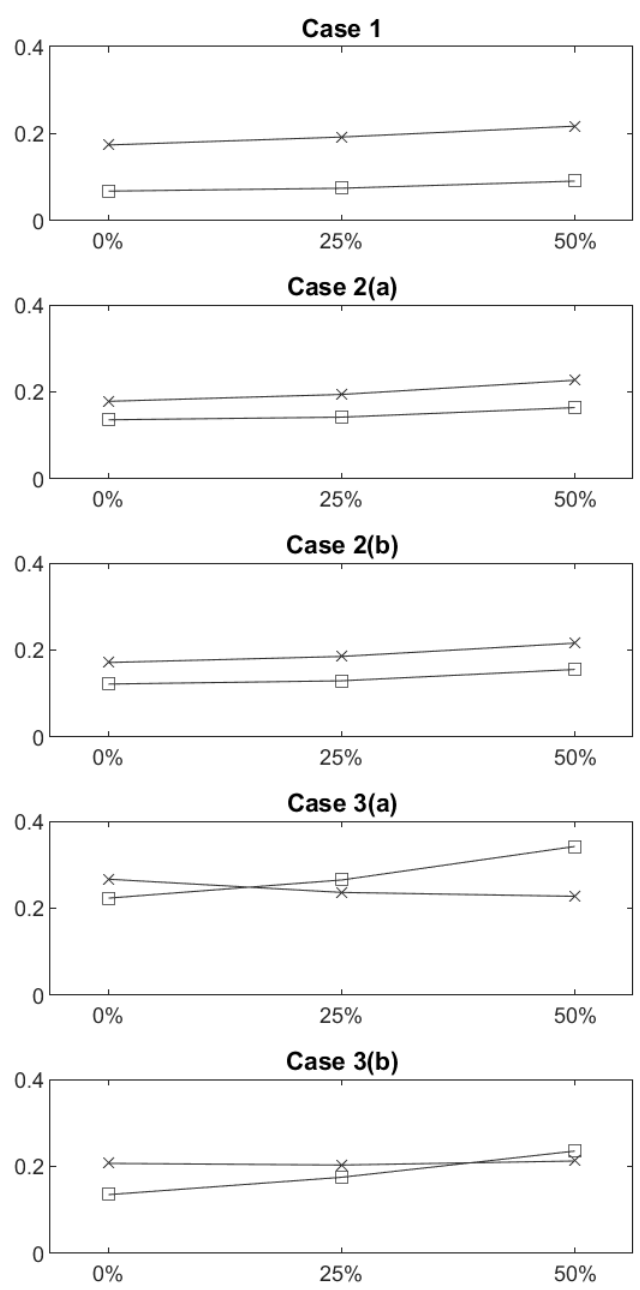

$\square$ DMDP Unadj

Figure 5.9: Median $L_{2}$ survival error under data cases 1-3 (5.3.9) was calculated for both the unadjusted DMDP-TR model and the Kaplan-Meier estimates. Median survival error is presented for both methods, separated by data case and censoring level. 

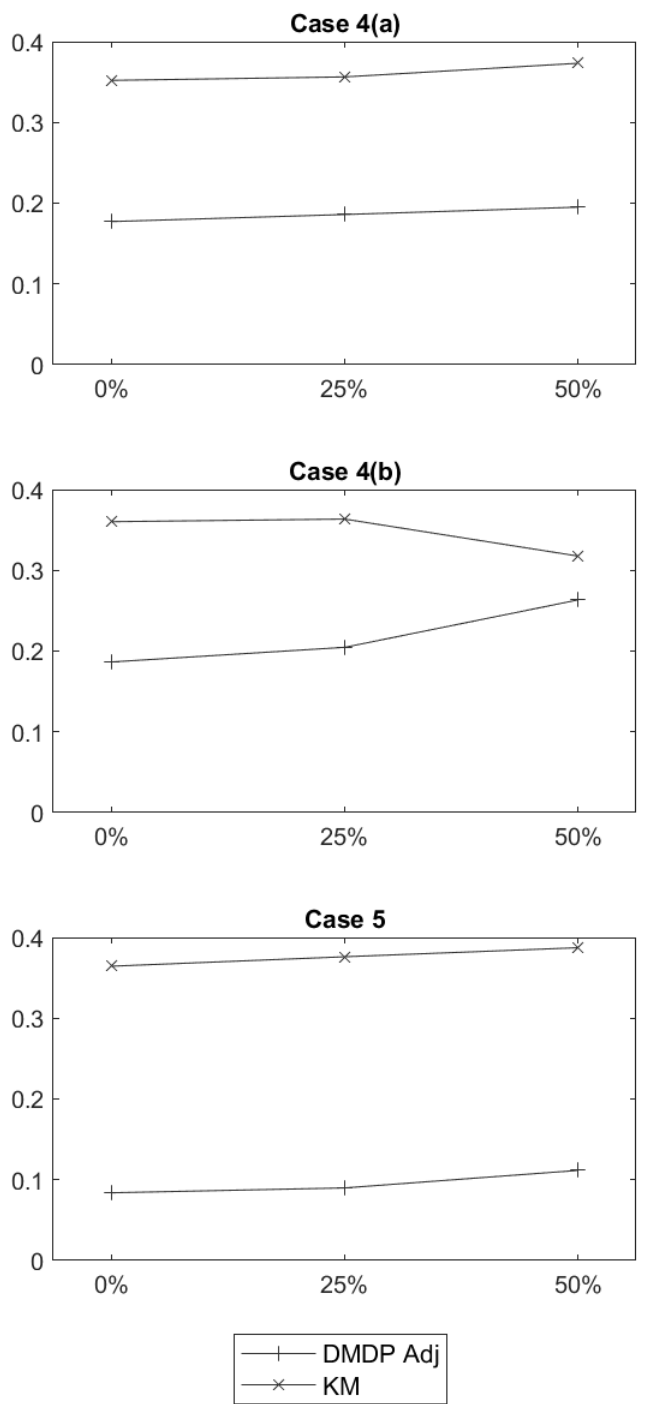

Figure 5.10: Median $L_{2}$ survival error under data cases 4 and 5 (5.3.10) was calculated for both the adjusted DMDP-TR model and the Kaplan-Meier estimates. Median survival error is presented for both methods, separated by data case and censoring level. 


\subsection{Data Example}

We close this chapter by demonstrating the use of the DMDP-TR model in analyzing the NTP urethane toxicology data presented in the preceding chapter. It was noted previously that there is strong biological reason to believe that these data violate proportional hazards due to an accumulating effect of treatment. This expectation was clearly justified insofar as the PH model fit the data poorly. In this light the NTP data offer a suitable example of the application of the DMDP-TR model in a controlled trial setting.

We analyzed the NTP data using the same six models and four hypotheses considered in the preceding simulation study. Event times are the time of death for individual mice and treatment is urethane exposure. For the three models capable of adjusting for a confounder, adjustment for baseline weight was included. While such covariate adjustment is not necessary due to the randomized design of the study, it is enlightening to demonstrate covariate adjustment in a real dataset. Due to the original expectation that urethane would be deleterious to subject health, the alternative hypothesis for the two Tarone tests is a negative trend in survival for increased treatment level.

Our analyses of the NTP data begins with hypothesis testing. P-values for all hypotheses of interest are included in Table 5.3. We can see that all methods conclude that there is a statistically significant effect of treatment. The two Tarone tests and the two logrank tests

of $H_{0}$ have negative test statistics indicating, as expected, that they estimate a negative association between treatment and survival. The two DMDP-TR models also estimate a negative relationship between treatment level and survival. This conclusion comes from considering the distribution of $E\left[\mu_{h i}\right]$ for each treatment group. From Figure 5.3 we can see that an increase in treatment is associated with a stochastic decrease in $E\left[\mu_{h i}\right]$. This indicates that increased treatment level results, on average, in an increase rate at which subjects lose health, and thus decreased survival times. It is also worth noting that the DMDP-TR models both reject the global null hypothesis of no treatment effect at much smaller $\alpha$-levels. One likely explanation for this phenomenon is the violation of proportional hazards, under which the log rank and Tarone tests exhibit decreased power. 
We next consider estimates of the Lowest Observed Effect Level (LOEL). Recalling that all six methods are adjusted for multiple comparisons, from Table 5.3 we can see that the two DMDP-TR models provide strong evidence that the lowest non-control group (10ppm) is the LOEL. The Tarone methods, on the other hand, are only able to conclude that the moderate treatment level $(30 \mathrm{ppm})$ has a statistically significant difference in survival compared to control at $\alpha=0.05$. The logrank tests, being two sided versions of the Tarone test, provide even weaker evidence and would only justify the conclusion that the highest treatment dose $(90 \mathrm{ppm})$ is associated with an adverse outcome. The DMDP-TR models also allow us to estimate the posterior probability that any given treatment is the LOEL; i.e., we can estimate the distribution of the LOEL. Table 5.4 provides estimates for the distribution of the LOEL from both the adjusted and unadjusted DMDP-TR models.

Finally, plots of estimated survival from both the DMDP-TR models and the KM method are included for reference in Figure 5.12. As expected, there is strong agreement between the DMDP-TR model estimates of survival and the Kaplan-Meier curves. It is worth noting once again that such agreement demonstrates that the proposed methodology provides survival estimation comparable to nonparametric methods in spite of being semi-parametric in nature.

\begin{tabular}{|l|llll|}
\hline Model & $H_{0}$ & $H_{c 2}$ & $H_{c 3}$ & $H_{c 4}$ \\
\hline DMDP-TR Unadj & $9.34 \times 10^{-45}$ & 0.022 & 0.003 & $9.34 \times 10^{-45}$ \\
DMDP-TR Adj & $4.87 \times 10^{-23}$ & 0.018 & 0.002 & $4.87 \times 10^{-23}$ \\
Tarone Unadj & $1.06 \times 10^{-11}$ & 0.087 & 0.014 & $1.06 \times 10^{-11}$ \\
Tarone Adj & $1.03 \times 10^{-11}$ & 0.094 & 0.010 & $1.03 \times 10^{-11}$ \\
Logrank Unadj & $2.11 \times 10^{-11}$ & 0.174 & 0.057 & $3.04 \times 10^{-11}$ \\
Logrank Adj & $2.06 \times 10^{-11}$ & 0.189 & 0.052 & $3.76 \times 10^{-11}$ \\
\hline
\end{tabular}

Table 5.3: The global null hypothesis and three treatment-vs-control null hypotheses were tested for six models of interest. P-values for these four hypotheses are presented for each model. 


\begin{tabular}{|l|llll|}
\hline & $P(\mathrm{LOEL}=2)$ & $P(\mathrm{LOEL}=3)$ & $P(\mathrm{LOEL}=4)$ & $P(\mathrm{LOEL}>4)$ \\
\hline DMDP-TR Unadj & 0.9777 & 0.0223 & 0.0001 & $9.3379 \times 10^{-45}$ \\
DMDP-TR Adj & 0.9823 & 0.0176 & $4.3363 \times 10^{-5}$ & $4.8739 \times 10^{-23}$ \\
\hline
\end{tabular}

Table 5.4: Posterior distribution of the LOEL estimated from both DMDP-TR models. 


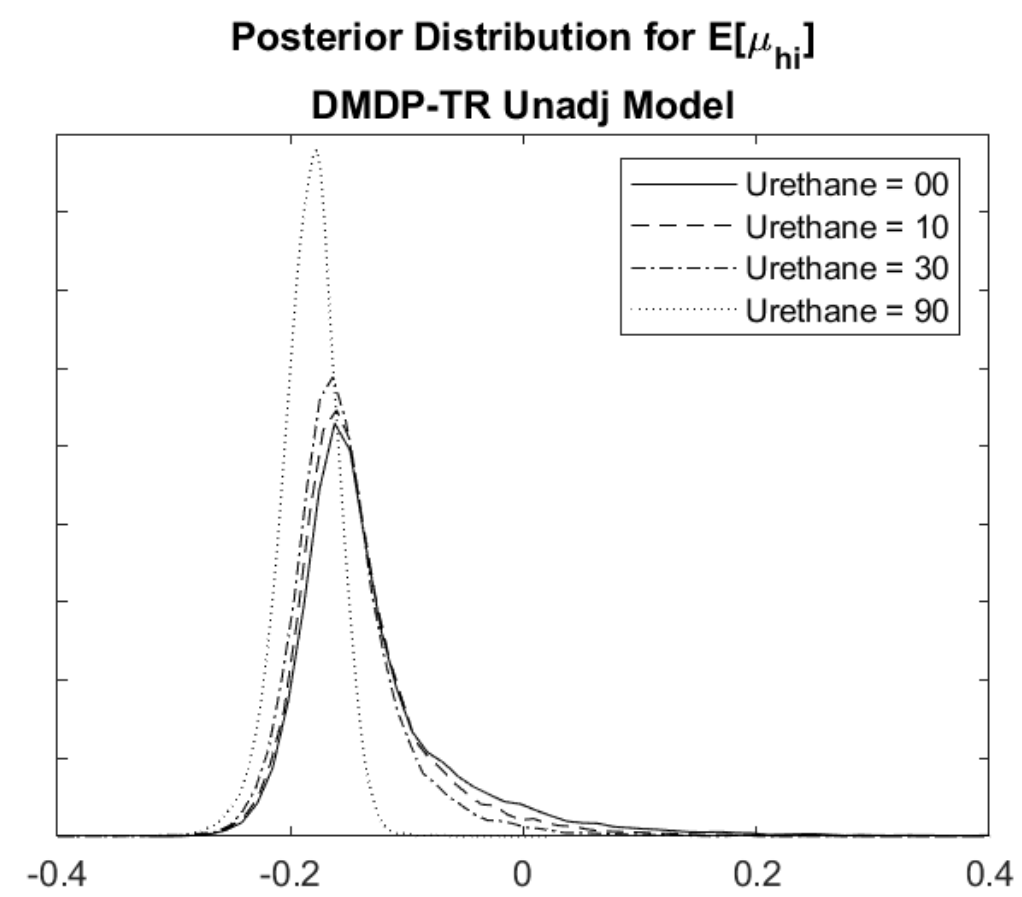

(a) DMDP-TR Unadjusted

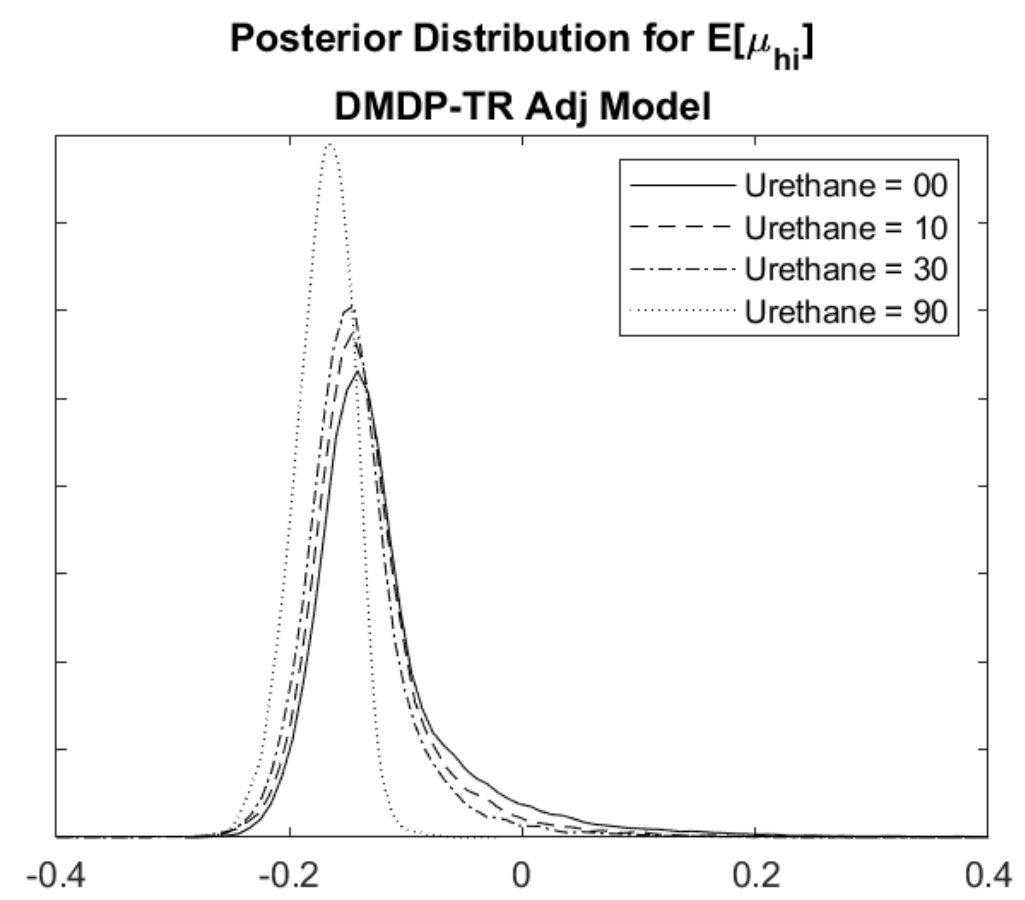

(b) DMDP-TR Adjusted

Figure 5.11: The posterior distribution of the treatment specific average drift term $E\left[\mu_{h i}\right]$ is presented for both the adjusted and unadjusted DMDP-TR models. 


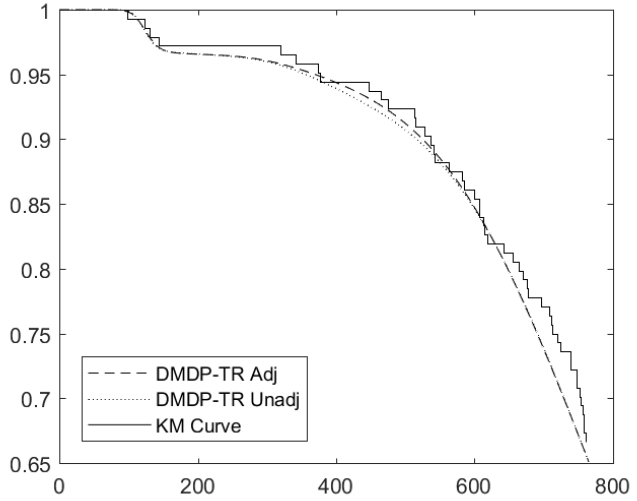

(a) Urethane $=00$

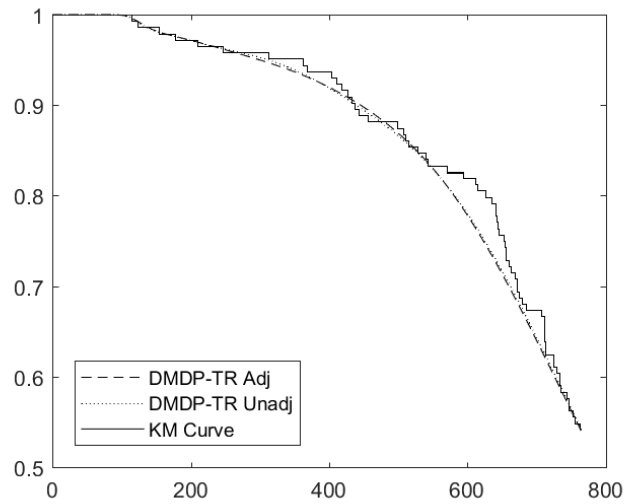

(c) Urethane $=30$

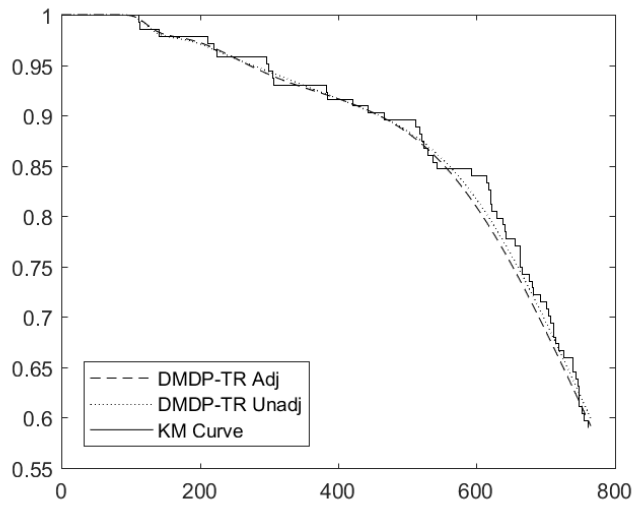

(b) Urethane $=10$

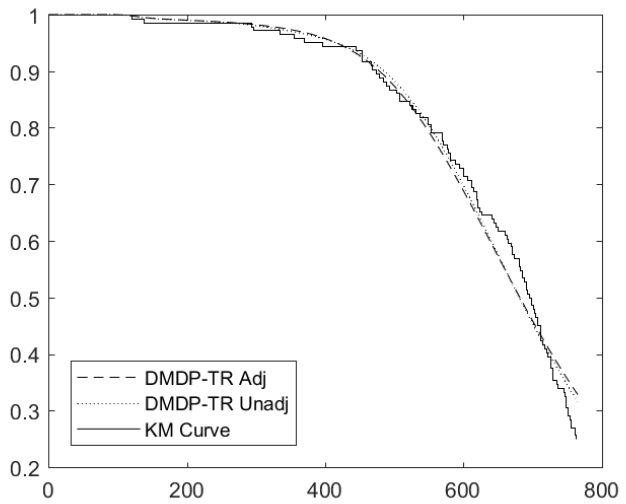

(d) Urethane $=90$

Figure 5.12: Estimated survival for the mice data conditional on urethane treatment level. Survival estimates are presented for both the adjusted and unadjusted DMDP-TR models. Kaplan-Meier estimates of the survival functions are included for reference.

\subsection{Discussion}

The current chapter has principally been concerned with the development of a flexible, semi-parametric Bayesian model capable of testing for an ordinal treatment effect on survival. Additionally, the developed methodology is able to adjust for confounding between treatment and subject health at baseline. We demonstrated via simulation study that the developed model outperforms standard methods in situations where the proportional hazards assumption is not appropriate. We also found that our model performs comparably to 
standard methods when data do in fact come from proportional hazards settings. In spite of this success there are several avenues of refinement related to the DMDP-TR model which are worth exploring.

The first point of potential refinement for the DMDP-TR model concerns the manner in which covariate adjustment is accomplished. As presented, the DMDP-TR model only allows non-treatment covariates to be associated with baseline health. This modeling decision was predicated on the motivation to adjust for confounding between treatment and subject health at time of treatment. There might, of course, be some scenarios where non-treatment covariates are more likely to affect subject health trend than initial subject health. As an example, some patients might be known to have a degenerative disease separate from the disease under study. Such subjects would generally be expected, on average, to have different slopes in health trend compared to those without the degenerative disease. It is unclear if assigning such covariates to the initial state regression structure in the proposed methodology would be sufficient. While we expect cases with health trend covariates to be the exception rather than the rule, further simulation studies exploring the performance of the DMDP-TR model would be advisable before use in such situations.

From the preceding work it is also unclear if the DMDP-TR model would be appropriate for settings where it makes more scientific sense for treatment to have an immediate effect on patient health. Corrective surgeries, ordered in terms of invasiveness, might be such a case. From our experience we expect that the DMDP-TR model would perform well at least in controlled trials settings where adjustment for potential confounders is unnecessary. Even when covariate adjustment is necessary, though, we would not be surprised if the proposed model performs adequately. Again, however, it would be advisable for further simulation studies be run before the DMDP-TR model is put to such use.

A final recommendation for further research relates to the DMDP model in general, and not to the TR context in particular. The NTP data used in this and the preceding chapter originally considered all combinations of two ordinal treatments, urethane and ethanol. We ignored ethanol in our example because previous analyses concluded that it had no statistically significant effect on survival. An extension of the DMDP methodology which 
would allow for modeling of two ordinal treatments and its application to TR would be an interesting line of inquiry. Dunson and Peddada (2008) propose a restricted dependent Dirichlet Process prior that might well be worth applying to TR for this purpose. They also discuss the possibility of a direct extension of the DMDP model to accommodate testing of multiple ordinal treatment levels. Both possibilities would be worthwhile potential extensions of the methodology discussed in this chapter. 


\section{Chapter 6 Summary AND CONCLUSiONS}

I am glad you are here with me. Here at the end of all things.

- J.R.R. Tolkien, The Return of the King

The preceding work was focused on developing and testing two novel methods for the analysis of time-to-event data. We conclude with a final chapter which will focus on reviewing these contributions, discussing their place in the broader landscape of survival analysis, and recommending avenues for further related research.

The Dirichlet Process Mixture for Threshold Regression (DPM-TR) model was developed as an alternative to common proportional hazards based methods. The motivation for this development was the recognition that hazard proportionality is often violated in common time-to-event settings where the effect of treatment accumulates over time, e.g. toxicology and pharmacology studies interested in chronic exposure. The parametric Threshold Regression model was seen as an ideal starting point in this endeavor for three major reasons. First, and perhaps most importantly, the Inverse Gaussian (IG) distribution on which TR is based has already received significant attention in both the survival and reliability literature. As a consequence, TR is already well understood from both a theoretical and an applied perspective. Secondly, TR makes assumptions on the hazard ratio which are clinically relevant in that it can distinguish between early and later effects of treatment. Lastly, model parameters related to baseline health and trend in health are easily interpretable for clinicians and other potential collaborators. 
The major drawback of TR is, of course, the strong parametric assumptions the model makes which increases the likelihood of poor model fit and severely limits the methods applicability. Much of the past research dedicated to remedying this shortcoming has focused on either Random Effect or Finite Mixture specifications, both of which have their own limitations. The solution proposed here was the DPM-TR model which addresses these issues and limitations via an infinite mixture model known as the Dirichlet Process mixture. Simulations and a data example demonstrated the usefulness of this model in analyzing time-to-event data which violate proportional hazards. The DPM-TR model should be ideally suited to datasets which are expected to violate proportional hazards and where regression analysis would be valuable.

There are, however, several areas where refinement to the DPM-TR model would be necessary for the methodology to be applicable more broadly. Two such cases which arise prominently in the survival literature are repeated measures and competing risks settings. The repeated measures setting could be addressed in a manner similar to the random effects model of Economou et al. (2015) (see Section 2.5.4) except that the random effects distribution would be assumed to be a draw from the DP instead of being assumed to be Gaussian. Such a model could be accomplished by appropriating the nested Dirichlet Process (nDP) methodology of Rodríguez, Dunson, and Gelfand (2008) which induces subject-level clustering by replacing the random atoms in the stick-breaking representation of the DP with random probability measures drawn from a DP. For example, if we have $M_{i}$ repeated observations of event times and covariates on subjects $i=1, \ldots, N$, i.e. $\left(T_{i m}, v_{i m}, w_{i m}\right)$ for $\left.m \in\left\{1, \ldots, M_{i}\right\}\right)$, then a nested DP mixture extension of the Economou et al. model might 
be specified, say, as follows:

$$
\begin{aligned}
T_{i m} \mid \mathbf{v}_{i m}, \mathbf{w}_{i m} \stackrel{i n d}{\sim} I G\left(x_{0 i m}, \mu_{i m}\right), \\
x_{0 i m}=\exp \left\{\mathbf{v}_{i m}^{\top} \boldsymbol{\alpha}_{i m}\right\}+\alpha_{n s}(m-1)+\alpha_{t} t_{i(m-1)}, \\
\mu_{i m}=\mathbf{w}_{i m}^{\top} \boldsymbol{\beta}_{i m}+\beta_{n s}(m-1)+\beta_{t} t_{i(m-1)}, \\
\theta_{i m}=\left[\begin{array}{l}
\boldsymbol{\alpha}_{i m} \\
\boldsymbol{\beta}_{i m}
\end{array}\right] \stackrel{i n d}{\sim} G_{i}, \\
G_{i} \sim D P\left(\gamma^{*} H^{*}\right), \\
H^{*} \sim \operatorname{DP}(\gamma H) .
\end{aligned}
$$

The above model utilizes regression structures on observation specific parameters $\left(x_{0 i m}, \mu_{i m}\right)$ which are analogous to those in the original Economou model and includes terms for time effects $\left(\alpha_{t}, \beta_{t}\right)$ and the number of past events $\left(\alpha_{n s}, \beta_{n s}\right)$. However, instead of imposing clustering via random intercepts terms as in the Economou model, the above model imposes subject-level clustering via subject-specific mixture distributions $G_{i}$ on the vector $\theta_{i m}$ of regression coefficients. We then assume the $G_{i}$ to be draws from a DP which, if we follow the nDP methodology specified by Rodriquez et al., could assume that the base measure $H^{*}$ is also a draw from the DP. The above model should capture the same relationships as the original Economou model with the additional benefit of increased flexibility due to the use of the DP.

Extension of the DPM-TR model to account for competing risks by jointly modeling multiple outcomes would also likely have substantial application. G. A. Whitmore (1986), Saebo et al. (2004), and Horrocks and Thompson (2004) present unique Threshold Regression based models which account for competing risks and which might easily be generalized to employ Dirichlet Process mixtures to account for more diverse heterogeneities.

A different extension of the DPM-TR model was also explored in this work. Specifically, the Dynamic Mixture of Dirichlet Processes for Threshold Regression (DMDP-TR) model was developed to analyze the effect of an ordinal treatment on time-to-event outcomes when the PH assumption is violated. This model was also specifically designed to account for 
potential confounding between treatment and covariates associated with baseline health. We were able to demonstrate that the DMDP-TR model was almost as powerful as existing methods in detecting a treatment effect under conditions where the $\mathrm{PH}$ assumption generally holds. Additionally, the proposed method is vastly more powerful in detecting treatment effects that violate $\mathrm{PH}$, both in the presence and absence of baseline health confounding.

In spite of this success, and as discussed at the end of Chapter 5, there are still several avenues of exploration and potential refinement with respect to the DMDP-TR model which might be worth exploring. The immediate questions which deserve attention regard the extent to which the DMDP-TR model can compensate for misspecification of the relationship between covariates and the TR parameters $\left(\mu_{i}\right.$ and $\left.x_{0 i}\right)$. Further avenues of refinement for the DMDP-TR model include development of a method for testing of multiple ordinal treatments. Of particular interest would be a model which facilitates testing for an interaction between the treatments of interest.

In summary, the preceding work has extended the applicability of TR in the analysis of time-to-event data. Ideally this work should act as a strong foundation for further research in generalizing TR methodologies to account for various model misspecification. 


\section{REFERENCES}

Aalen, O., Borgan, O., \& Gjessing, H. (2008). Survival and Event History Analysis: A Process Point of View. Springer New York. Retrieved from https://books.google .com/books? id=2toprArSUMAC

Aalen, O., \& Gjessing, H. (2001, 02). Understanding the shape of the hazard rate: a process point of view (with comments and a rejoinder by the authors). Statist. Sci., 16(1), 1-22. Retrieved from https://doi.org/10.1214/ss/998929473 doi: 10.1214/ss/ 998929473

Andersen, P. K., Klein, J. P., Knudsen, K. M., \& y Palacios, R. T. (1997). Estimation of variance in Cox's regression model with shared gamma frailties. Biometrics, 53(4), $1475-1484$.

Balka, J., Desmond, A., \& McNicholas, P. (2011). Bayesian and likelihood inference for cure rates based on defective inverse Gaussian regression models. Journal of Applied Statistics, 38(1), 127-144. Retrieved from https://ideas.repec.org/a/taf/ japsta/v38y2011i1p127-144.html doi: 10.1080/02664760903271940

Berger, J., \& Delampady, M. (1987, 08). Testing precise hypotheses. Statist. Sci., 2(3), 317-335. Retrieved from https://doi.org/10.1214/ss/1177013238 doi: 10.1214/ ss $/ 1177013238$

Blackwell, D., \& MacQueen, J. B. (1973, 03). Ferguson distributions via Polya urn schemes. Ann. Statist., 1(2), 353-355. Retrieved from https://doi.org/10.1214/ aos/1176342372 doi: 10.1214/aos/1176342372

Bouliotis, G., \& Billingham, L. (2011, 12). Crossing survival curves: alternatives to the 
log-rank test. Trials, 12 Suppl 1, A137. doi: 10.1186/1745-6215-12-S1-A137

Bretz, F., Maurer, W., Brannath, W., \& Posch, M. (2009). A graphical approach to sequentially rejective multiple test procedures. Statistics in Medicine, 28(4), 586-604. Retrieved from https://onlinelibrary.wiley.com/doi/abs/10.1002/sim.3495 doi: $10.1002 / \operatorname{sim} .3495$

Bush, C., \& MacEachern, S. (1996). A semiparametric Bayesian model for randomised block designs. Biometrika, 83(2), 275-285. Retrieved from http://dx.doi.org/10.1093/ biomet/83.2.275 doi: 10.1093/biomet/83.2.275

Caroni, C. (2017). First Hitting Time Regression Models. Wiley-Blackwell. Retrieved from https://onlinelibrary.wiley.com/doi/abs/10.1002/9781119437260.ch2 doi: 10.1002/9781119437260.ch2

Chen, Y.-I. (2000, 03). Multiple comparisons in carcinogenesis study with rightcensored survival data. Statistics in Medicine, 19, 353-67. doi: 10.1002/(SICI)1097 -0258(20000215)19:33.0.CO;2-B

Chhikara, R. S., \& Folks, L. J. (1989). The Inverse Gaussian Distribution: Theory, Methodology, and Applications. New York, NY, USA: Marcel Dekker, Inc.

Christensen, R., \& Johnson, W. (1988). Modelling accelerated failure time with a Dirichlet process. Biometrika, 75(4), 693-704. Retrieved from http://www.jstor.org/ stable/2336310

Cox, D. R. (1972). Regression models and life-tables. Journal of the Royal Statistical Society. Series B (Methodological), 34(2), 187-220. Retrieved from http://www.jstor.org/ stable/2985181

Damlen, P., Wakefield, J., \& Walker, S. (1999). Gibbs sampling for Bayesian non-conjugate and hierarchical models by using auxiliary variables. Journal of the Royal Statistical Society Series B, 61(2), 331-344. Retrieved from https://EconPapers.repec.org/ RePEc:bla:jorssb:v:61:y:1999:i:2:p:331-344

Dempster, A. P., Laird, N. M., \& Rubin, D. B. (1977). Maximum likelihood from incomplete data via the EM algorithm. Journal of the Royal Statistical Society. Series B (Methodological), 39(1), 1-38. Retrieved from http://www.jstor.org/stable/2984875 
Dunson, D. (2006, 02). Bayesian dynamic modeling of latent trait distributions. Biostatistics, 7(4), 551-568. doi: 10.1093/biostatistics/kxj025

Dunson, D., \& Peddada, S. (2008). Bayesian nonparametric inference on stochastic ordering. Biometrika, 95(4), 859-874. Retrieved from http://dx.doi.org/10.1093/biomet/ asn043 doi: 10.1093/biomet/asn043

Eaton, W., \& Whitmore, G. (1977, 1 1). Length of stay as a stochastic process: A general approach and application to hospitalization for schizophrenia. Journal of Mathematical Sociology, 5(2), 273-292. doi: 10.1080/0022250X.1977.9989877

Eberly, L. E., Grambsch, P., \& Connett, J. E. (2001). Understanding the shape of the hazard rate: A process point of view: Comment. Statistical Science, 16(1), 16-19. Retrieved from http://www.jstor.org/stable/2676775

Economou, P., Malefaki, S., \& Caroni, C. (2015). Bayesian threshold regression model with random effects for recurrent events. Methodology and Computing in Applied Probability, 17(4), 871-898. Retrieved from https://doi.org/10.1007/s11009-015 -9445-8 doi: 10.1007/s11009-015-9445-8

Escobar, M., \& West, M. (1998, 08). Computing nonparametric hierarchical models. Practical Nonparametric and Semiparametric Bayesian Statistics, 133.

Ferguson, T., \& Phadia, E. (1979). Bayesian nonparametric estimation based on censored data. The Annals of Statistics, 7(1), 163-186.

Gelfand, A., \& Dey, D. (1994). Bayesian model choice: Asymptotics and exact calculations. Journal of the Royal Statistical Society. Series B (Methodological), 56(3), 501-514. Retrieved from http://www.jstor.org/stable/2346123

Gelfand, A., \& Smith, A. (1990). Sampling-based approaches to calculating marginal densities. Journal of the American Statistical Association, 85(410), 398-409. Retrieved from http://www.jstor.org/stable/2289776

Gelman, A., Carlin, J. B., Stern, H. S., Dunson, D., Vehtari, A., \& Rubin, D. (2013). Bayesian Data Analysis (3rd ed.). Taylor \& Francis. Retrieved from https://books .google.com/books?id=ZXL6AQAAQBAJ

Hans, C., \& Dunson, D. (2005). Bayesian inferences on umbrella orderings. Biometrics, 61 
4, 1018-26.

Hanson, T. (2006). Modeling censored lifetime data using a mixture of gammas baseline. Bayesian Anal., 1(3), 575-594. Retrieved from https://doi.org/10.1214/06-BA119 doi: 10.1214/06-BA119

Hanson, T., \& Johnson, W. O. (2004). A Bayesian semiparametric aft model for interval-censored data. Journal of Computational and Graphical Statistics, 13(2), 341-361. Retrieved from https://doi.org/10.1198/1061860043489 doi: 10.1198/ 1061860043489

Hastie, T., \& Tibshirani, R. (1990). Exploring the nature of covariate effect in the proportional hazards model. Biometrics, 46, 1005-1016.

Holm, S. (1979). A simple sequentially rejective multiple test procedure. Scandinavian Journal of Statistics, 6(2), 65-70. Retrieved from http://www.jstor.org/stable/ 4615733

Horrocks, J., \& Thompson, M. E. (2004, Mar 01). Modeling event times with multiple outcomes using the Wiener process with drift. Lifetime Data Analysis, 10(1), 2949. Retrieved from https://doi.org/10.1023/B:LIDA.0000019254.29153.1a doi: 10.1023/B:LIDA.0000019254.29153.1a

Hothorn, T., Hornik, K., van de Wiel, M. A., \& Zeileis, A. (2008). Implementing a class of permutation tests: The coin package. Journal of Statistical Software, 28(8), 1-23. Retrieved from http://www.jstatsoft.org/v28/i08/

Ibrahim, J., Chen, M.-H., \& Sinha, D. (2001). Bayesian Survival Analysis. Springer.

Ishwaran, H., \& James, L. F. (2001). Gibbs sampling methods for stick-breaking priors. Journal of the American Statistical Association, 96(453), 161-173. Retrieved from https://doi.org/10.1198/016214501750332758 doi: 10.1198/ 016214501750332758

Ishwaran, H., \& James, L. F. (2002). Approximate Dirichlet process computing in finite normal mixtures: Smoothing and prior information. Journal of Computational and Graphical Statistics, 11(3), 508-532. Retrieved from http://www.jstor.org/ stable/1391111 
Ishwaran, H., \& Zarepour, M. (2000). Markov chain Monte Carlo in approximate Dirichlet and beta two-parameter process hierarchical models. Biometrika, 87(2), 371-390. Retrieved from http://dx.doi.org/10.1093/biomet/87.2.371 doi: 10.1093/biomet/ 87.2 .371

Ishwaran, H., \& Zarepour, M. (2002). Exact and approximate sum representations for the Dirichlet process. The Canadian Journal of Statistics / La Revue Canadienne de Statistique, 30(2), 269-283. Retrieved from http://www.jstor.org/stable/ 3315951

Johnson, A., Jones, G., \& Neath, R. (2013, 08). Component-wise Markov chain Monte Carlo: Uniform and geometric ergodicity under mixing and composition. Statist. Sci., 28(3), 360-375. Retrieved from https://doi.org/10.1214/13-STS423 doi: 10.1214/13-STS423

Kass, R., \& Raftery, A. (1995). Bayes factors. Journal of the American Statistical Association, 90(430), 773-795. Retrieved from https://www.tandfonline.com/doi/abs/ 10.1080/01621459.1995.10476572 doi: 10.1080/01621459.1995.10476572

Klein, J., \& Moeschberger, M. (2003). Survival analysis: Methods for censored and truncated data. Springer.

Kottas, A. (2006, 03). Nonparametric Bayesian survival analysis using mixtures of Weibull distributions. Journal of Statistical Planning and Inference, 136, 578-596.

Kuo, L., \& Smith, A. (1992). Bayesian computations in survival models via the Gibbs sampler. In J. P. Klein \& P. K. Goel (Eds.), Survival Analysis: State of the Art (p. 11-24). Dordrecht: Springer Netherlands.

Lancaster, T. (1972). A stochastic model for the duration of a strike. Journal of the Royal Statistical Society. Series A (General), 135(2), 257-271. Retrieved from http:// wWw.jstor.org/stable/2344321

Lee, M., Chang, M., \& Whitmore, G. (2008). A threshold regression mixture model for assessing treatment efficacy in a multiple myeloma clinical trial. Journal Of Biopharmaceutical Statistics, $18(6), 1136$ - 1149.

Lee, M., Shubina, M., \& Zaslavsky, A. (2005). Bayesian analysis for markers and degra- 
dation. Retrieved from https://www.researchgate.net/publication/242072623 Bayesian_Analysis_for_Markers_and_Degradation

Lee, M., \& Whitmore, G. $(2006,11)$. Threshold regression for survival analysis: Modeling event times by a stochastic process reaching a boundary. Statistical Science, 21(4), 501-513. doi: 10.1214/088342306000000330

Li, H., Han, D., Hou, Y., Chen, H., \& Chen, Z. (2015, 01). Statistical inference methods for two crossing survival curves: A comparison of methods. PLOS ONE, 10, e0116774. doi: 10.1371/journal.pone.0116774

Lin, X., \& Wang, H. (2004). A new testing approach for comparing the overall homogeneity of survival curves. Biometrical Journal: Journal of Mathematical Methods in Biosciences, 46(5), 489-496.

Lin, X., \& Xu, Q. (2010). A new method for the comparison of survival distributions. Pharmaceutical Statistics, 9(1), 67-76. Retrieved from https://onlinelibrary.wiley .com/doi/abs/10.1002/pst.376 doi: 10.1002/pst.376

Liu, K., Qiu, P., \& Sheng, J. (2007). Comparing two crossing hazard rates by Cox proportional hazards modelling. Statistics in Medicine, 26(2), 375-391.

Logan, B., Wang, H., \& Zhang, M.-J. (2005). Pairwise multiple comparison adjustment in survival analysis. Statistics in Medicine, 24 (16), 2509-2523. Retrieved from https:// onlinelibrary.wiley.com/doi/abs/10.1002/sim.2125 doi: 10.1002/sim.2125

MacEachern, S. N. (1999). Dependent Dirichlet processes. Proceedings of the Bayesian Statistical Science Section.

MacEachern, S. N., \& Mueller, P. (1998). Estimating mixture of Dirichlet process models. Journal of Computational and Graphical Statistics, 7(2), 223-238. Retrieved from https://www.tandfonline.com/doi/abs/10.1080/10618600.1998.10474772 doi: 10.1080/10618600.1998.10474772

Marcus, R., Peritz, E., \& Gabriel, K. (1976). On closed testing procedures with special reference to ordered analysis of variance. Biometrika, 63(3), 655-660. Retrieved from http://www.jstor.org/stable/2335748

McGilchrist, C. A., \& Aisbett, C. W. (1991). Regression with frailty in survival analysis. 
Biometrics, 47(2), 461-466.

Nelder, J. A., \& Wedderburn, R. W. M. (1972). Generalized linear models. Journal of the Royal Statistical Society. Series A (General), 135(3), 370-384. Retrieved from http://www.jstor.org/stable/2344614

Nickerson, R. (2000). Null hypothesis significance testing: A review of an old and continuing controversy. Psychological Methods, 5(2), 241-301.

Pennell, M., \& Dunson, D. (2008, 07). Nonparametric Bayes testing of changes in a response distribution with an ordinal predictor. , 64, 413-23.

Pennell, M., Whitmore, G. A., \& Lee, M.-L. T. (2010). Bayesian random-effects threshold regression with application to survival data with nonproportional hazards. Biostatistics, 11(1), 111-126. Retrieved from http://dx.doi.org/10.1093/biostatistics/ kxp041 doi: 10.1093/biostatistics/kxp041

Pettit, L., \& Young, K. (1999). Bayesian analysis for inverse Gaussian lifetime data with measures of degradation. Journal of Statistical Computation and Simulation, 63(3), 217-234. doi: 10.1080/00949659908811954

Qiu, P., \& Sheng, J. (2008). A two-stage procedure for comparing hazard rate functions. Journal of the Royal Statistical Society: Series B (Statistical Methodology), 70(1), $191-208$.

R Core Team. (2018). R: A language and environment for statistical computing [Computer software manual]. Vienna, Austria. Retrieved from https://www.R-project.org/

Roberts, G. O., \& Rosenthal, J. S. (2001). Optimal scaling for various Metropolis-Hastings algorithms. Statistical Science, 16(4), 351-367. Retrieved from http://www.jstor .org/stable/3182776

Rodríguez, A., Dunson, D. B., \& Gelfand, A. E. (2008). The nested Dirichlet process. Journal of the American Statistical Association, 103(483), 1131-1154. Retrieved from https://doi.org/10.1198/016214508000000553 doi: 10.1198/ 016214508000000553

Rohmeyer, K., \& Klinglmueller, F. (2018). gMCP: Graph based multiple test procedures [Computer software manual]. Retrieved from https://cran.r-project.org/ 
package $=\mathrm{gMCP} \quad(\mathrm{R}$ package version $0.8-14)$

Ross, S. M. (2006). Introduction to Probability Models, Ninth Edition. Orlando, FL, USA: Academic Press, Inc.

Saebo, S., Almoy, T., \& Aastveit, A. (2004). Disease resistance modelled as first-passage times of genetically dependent stochastic processes. Journal of the Royal Statistical Society: Series C (Applied Statistics), 54(1), 273-285.

Shirley, E. (1977). A non-parametric equivalent of Williams' test for contrasting increasing dose levels of a treatment. Biometrics, 33(2), 386-389. Retrieved from http:// WwW.jstor.org/stable/2529789

Stogiannis, D., \& Caroni, C. (2013). Issues in fitting inverse Gaussian first hitting time regression models for lifetime data. Communications in Statistics - Simulation and Computation, 42(9), 1948-1960. Retrieved from https://doi.org/10.1080/ 03610918.2012.687061 doi: 10.1080/03610918.2012.687061

Stone, M. (1977). An asymptotic equivalence of choice of model by cross-validation and akaike's criterion. Journal of the Royal Statistical Society. Series B (Methodological), 39(1), 44-47. Retrieved from http://www.jstor.org/stable/2984877

Susarla, V., \& Ryzin, J. V. (1976). Nonparametric Bayesian estimation of survival curves from incomplete observations. Journal of the American Statistical Association, 71 (356), 897-902. doi: 10.1080/01621459.1976.10480966

Tarone, R. (1975, 12). Tests for trend in life table analysis. Biometrika, 62(3), 679-690. Retrieved from https://doi.org/10.1093/biomet/62.3.679 doi: 10.1093/biomet/ 62.3 .679

Tarone, R., \& Ware, J. (1977, 04). On distribution-free tests for equality of survival distributions. Biometrika, 64(1), 156-160. Retrieved from https://doi.org/10.1093/ biomet/64.1.156 doi: 10.1093/biomet/64.1.156

Tian, L., Zucker, D., \& Wei, L. J. (2005). On the Cox model with time-varying regression coefficients. Journal of the American Statistical Association, 100, 172-183.

Tweedie, M. C. K. (1957, 06). Statistical properties of inverse Gaussian distributions. i. Ann. Math. Statist., 28(2), 362-377. Retrieved from https://doi.org/10.1214/ 
aoms/1177706964 doi: 10.1214/aoms/1177706964

Upadhyay, S. K., Agrawal, R., \& Smith, A. (1996). Bayesian analysis of inverse Gaussian non-linear regression by simulation. Sankhya: The Indian Journal of Statistics, Series B (1960-2002), 58(3), 363-378. Retrieved from http://www.jstor.org/stable/ 25052963

USDHHS. (2004). NTP technical report on the toxicology and carcinogenesis studies of urethane, ethanol, and urethane/ethanol.

Walker, S. G., \& Mallick, B. K. (1997). Hierarchical generalized linear models and frailty models with Bayesian nonparametric mixing. Journal of the Royal Statistical Society: Series B (Statistical Methodology), 59(4), 845-860. doi: 10.1111/1467-9868.00101

West, M., Mueller, P., \& Escobar, M. D. (1994). Hierarchical priors and mixture models, with application in regression and density estimation. Aspects of Uncertainty: A Tribute to D. V. Lindley, 363-386.

Westfall, P. (1997). Multiple testing of general contrasts using logical constraints and correlations. Journal of the American Statistical Association, 92(437), 299-306. Retrieved from https://doi.org/10.1080/01621459.1997.10473627 doi: 10.1080/ 01621459.1997.10473627

Westfall, P., Johnson, W., \& Utts, J. (1997, 06). A Bayesian perspective on the Bonferroni adjustment. Biometrika,84(2), 419-427. Retrieved from https://doi.org/10.1093/ biomet/84.2.419 doi: 10.1093/biomet/84.2.419

Whitmore, G. (1975). The inverse Gaussian distribution as a model of hospital stay. Health Services Research, 10(3), 297-302.

Whitmore, G. (1986). Normal-gamma mixtures of inverse Gaussian distributions. Scandinavian Journal of Statistics, 13(3), 211-220. Retrieved from http://www.jstor.org/ stable/4616027

Whitmore, G., Crowder, M., \& Lawless, J. (1998, Aug 01). Failure inference from a marker process based on a bivariate Wiener model. Lifetime Data Analysis, 4 (3), 229251. Retrieved from https://doi.org/10.1023/A:1009617814586 doi: 10.1023/A: 1009617814586 
Whitmore, G., \& Su, Y. (2007, Jun 01). Modeling low birth weights using threshold regression: results for U.S. birth data. Lifetime Data Analysis, 13(2), 161190. Retrieved from https://doi.org/10.1007/s10985-006-9032-y doi: 10.1007/ s10985-006-9032-y

Whitmore, G. A. (1986). First-passage-time models for duration data: Regression structures and competing risks. Journal of the Royal Statistical Society. Series D (The Statistician), 35(2), 207-219. Retrieved from http://www.jstor.org/stable/2987525

Wiens, B., \& Dmitrienko, A. (2005). The fallback procedure for evaluating a single family of hypotheses. Journal of Biopharmaceutical Statistics, 15(6), 929-942. Retrieved from https://doi.org/10.1080/10543400500265660 (PMID: 16279352) doi: 10.1080/ 10543400500265660

Williams, D. (1986). A note on Shirley's nonparametric test for comparing several dose levels with a zero-dose control. Biometrics, 42(1), 183-186. Retrieved from http:// wWw . jstor .org/stable/2531254 